NUREG.CR-4772

SAND86 - 1996

RX, AN

Printed February 1987
Received by OSTI

JUN 181987

\title{
Accident Sequence Evaluation Program Human Reliability Analysis Procedure
}

Alan D. Swain

Histi:...

Prepared by

Sandia National Laboratories

Albuquerque, New Mexico 87185 and Livermore, California 94550

for the United States Department of Energy

under Contract DE-AC04-76DP00789
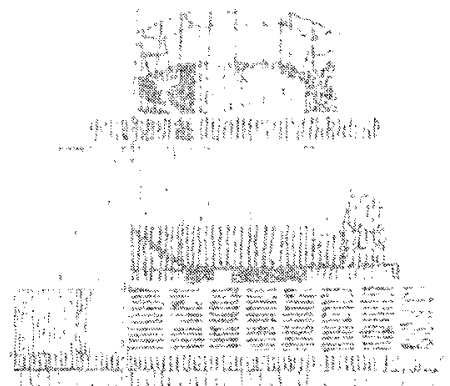

Prepared for

U. S. NUCLEAR REGULATORY COMMISSION 


\section{DISCLAIMER}

This report was prepared as an account of work sponsored by an agency of the United States Government. Neither the United States Government nor any agency Thereof, nor any of their employees, makes any warranty, express or implied, or assumes any legal liability or responsibility for the accuracy, completeness, or usefulness of any information, apparatus, product, or process disclosed, or represents that its use would not infringe privately owned rights. Reference herein to any specific commercial product, process, or service by trade name, trademark, manufacturer, or otherwise does not necessarily constitute or imply its endorsement, recommendation, or favoring by the United States Government or any agency thereof. The views and opinions of authors expressed herein do not necessarily state or reflect those of the United States Government or any agency thereof. 


\section{DISCLAIMER}

Portions of this document may be illegible in electronic image products. Images are produced from the best available original document. 


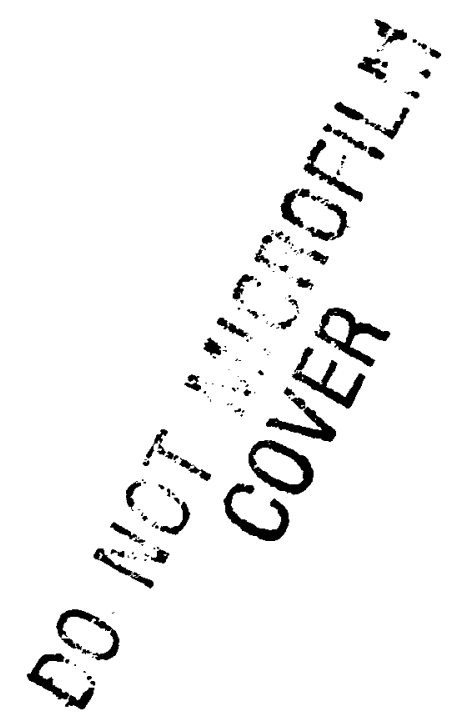

NOTICE

This report was prepared as an account of work sponsored by an agency of the United States Government. Neither the United States Government nor any agency thereof, or any of their employees, makes any warranty, expressed or implied, or assumes any legal liability or responsibility for any third party's use, or the results of such use, of any information, apparatus product or process disclosed in this report, or represents that its use by such third party would not infringe privately owned rights.

Available from

Superintendent of Documents

U.S. Government Printing Office

Post Office Box 37082

Washington, D.C. 20013-7082

and

National Technical Information Service

Springfield, VA 22161

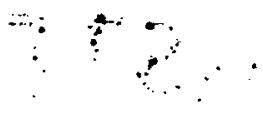




\author{
NUREG/CR-4772 \\ SAND86-1996 \\ $\mathrm{RX}, \mathrm{AN}$
}

TI87 010664

\title{
ACCIDENT SEQUENCE EVALUATION PROGRAM HUMAN RELIABILITY ANALYSIS PROCEDURE
}

\author{
February 1987
}

\author{
Alan D. Swain \\ Sandia National Laboratories \\ Albuquerque, NM 87185 \\ operated by Sandia Corporation \\ for the \\ U.S. Department of Energy
}

\section{This document is}

PUBLICLY REYEASABLE

\author{
Prepared for \\ Division of Reactor System Safety \\ office of Nuclear Regulatory Research \\ U.S. Nuclear Regulatory Commission \\ Washington, D.C. 20555 \\ Under Memorandum of Understanding DOE 40-550-75 \\ NRC FIN A1228
}

\section{DISCLAIMER}

This report was prepared as an account of work sponsored by an agency of the United States Government. Neither the United States Government nor any agency thereof, nor any of their employees, makes any warranty, express or implied, or assumes any legal liability or responsibility for the accuracy, completeness, or usefulness of any information, apparatus, product, or process disclosed, or represents that its use would not infringe privately owned rights. Reference herein to any specific commercial product, process, or service by trade name, trademark, manufacturer, or otherwise does not necessarily constitute or imply its endorsement, recommendation, or favoring by the United States Government or any agency thereof. The views and opinions of authors expressed herein do not necessarily state or reflect those of the United States Government or any agency thereof. 


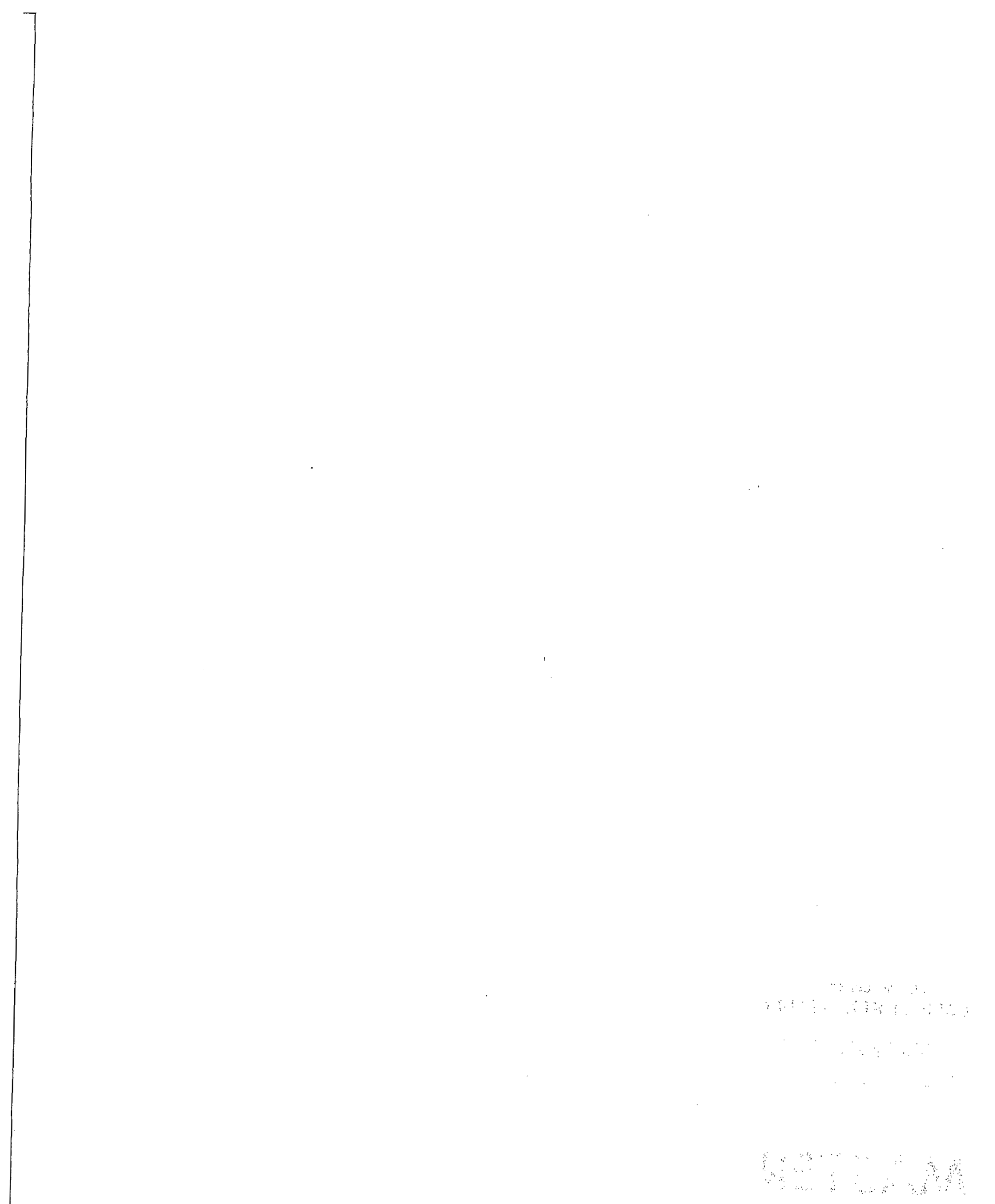


This document presents a shortened version of the procedure, models, and data for human reliability analysis (HRA) which are presented in the Handbook of Human Reliability Analysis With Emphasis on Nuclear Power Plant Applications (NUREG/CR-1278, August 1983). This shortened version was prepared and tried out as part of the Accident Sequence Evaluation Program (ASEP) funded by the U.S. Nuclear Regulatory Commission and managed by Sandia National Laboratories. The intent of this new HRA procedure, called the "ASEP HRA Procedure," is to enable systems analysts, with minimal support from experts in human reliability analysis, to make estimates of human error probabilities and other human performance characteristics which are sufficiently accurate for many probabilistic risk assessments. The ASEP HRA Procedure consists of a Pre-Accident Screening HRA, a Pre-Accident Nominal HRA, a Post-Accident Screening HRA, and a Post-Accident Nominal HRA. The procedure in this document includes changes made after tryout and evaluation of the procedure in four nuclear power plants by four different systems analysts and related personnel, including human reliability specialists. The changes consist of some additional explanatory material (including examples), and more detailed definitions of some of the terms. 

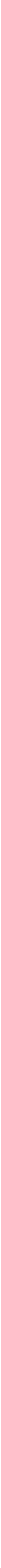


\begin{tabular}{|c|c|}
\hline & page \\
\hline Acknowledgments & ix \\
\hline List of Abbreviations & $\mathrm{x}$ \\
\hline Definitions of Technical Terms & $\mathrm{xi}$ \\
\hline Executive Summary & xxiii \\
\hline Part I Overview & $I-1$ \\
\hline $\begin{array}{l}\text { Chapter } 1 \text { Introduction } \\
\text { Purpose and Background of the ASEP HRA Procedure } \\
\text { Scope } \\
\text { Organization of the Report } \\
\text { How to Use This Report } \\
\text { Plant/Simulator Visit Procedure for Obtaining Information } \\
\text { for ASEP HRAs }\end{array}$ & $\begin{array}{l}1-1 \\
1-1 \\
1-3 \\
1-4 \\
1-5 \\
1-6\end{array}$ \\
\hline $\begin{array}{l}\text { Chapter } 2 \text { Concepts, Assumptions, and Limitations } \\
\text { Usefulness of Human Reliability Analysis } \\
\text { HEPs and their UCBs } \\
\text { Starting Point for the ASEP HRA Procedure } \\
\text { Screening and Nominal HRAs } \\
\text { Use of Sensitivity Analysis } \\
\text { Recovery Factors } \\
\text { Skill-Based, Rule-Based, and Knowledge-Based Behavior } \\
\text { Misdiagnosis }\end{array}$ & $\begin{array}{l}2-1 \\
2-1 \\
2-1 \\
2-2 \\
2-2 \\
2-4 \\
2-4 \\
2-5 \\
2-5\end{array}$ \\
\hline Part II ASEP HRA Procedure for Pre-Accident Tasks & II -1 \\
\hline $\begin{array}{l}\text { Chapter } 3 \text { General Information for HRA Procedure for Pre-Accident Tasks } \\
\text { Definitions of Terms } \\
\text { Pre-Accident Tasks of Interest } \\
\text { A Basic HEP for Pre-Accident Tasks } \\
\text { Recovery Factors for Pre-Accident HRA } \\
\text { Dependence Effects for Pre-Accident HRA } \\
\text { An Example Illustrating Dependence Among Human Actions }\end{array}$ & $\begin{array}{l}3-1 \\
3-1 \\
3-3 \\
3-4 \\
3-4 \\
3-5 \\
3-7\end{array}$ \\
\hline $\begin{array}{l}\text { Chapter } 4 \text { ASEP Screening HRA for Pre-Accident Tasks } \\
\text { General Information } \\
\text { The Procedure }\end{array}$ & $\begin{array}{l}4-1 \\
4-1 \\
4-1\end{array}$ \\
\hline $\begin{array}{l}\text { Chapter } 5 \text { ASEP Nominal HRA for Pre-Accident Tasks } \\
\text { General Information } \\
\text { Discussion of ASEP Dependence Model } \\
\text { The Procedure }\end{array}$ & $\begin{array}{l}5-1 \\
5-1 \\
5-2 \\
5-5\end{array}$ \\
\hline
\end{tabular}


Chapter 6 General Information for HRA Procedure for Post-Accident Tasks 6-1 Definitions of Terms 6-1

Chapter 7 ASEP Screening HRA for Post-Accident Tasks 7-1 General Information $\quad 7-1$ The Procedure $7-1$

A Hypothetical Example of a Screening HRA $7-9$

Chapter 8 ASEP Nominal HRA for Post-Accident Tasks $8-1$ General Information $8-1$ The Procedure $\quad 8-2$

A Hypothetical Example of a Nominal HRA $\quad 8-15$

Part IV Concluding Comments $\quad$ IV-1

Chapter 9 Results of Tryouts of the ASEP HRA Procedure 9-1 Overview $9-1$

The Tryouts $9-1$

Tryouts of the ASEP HRA Procedure for Pre-Accident Tasks 9-2

Tryouts of the ASEP HRA Procedure for Post-Accident Tasks 9-3

A Comparison of Estimated HEPs Using ASEP HRA Procedure and $9-5$ THERP/Handbook

$\begin{array}{lll}\text { Chapter } 10 \text { Conclusion } & 10-1\end{array}$

$\begin{array}{lr}\text { References } & 11-1\end{array}$

Appendix A Relationship of ASEP HRA Procedure to RMIEP Screening Rules A-1

Appendix B Computer Program for Propagating Uncertainty Bounds B-1 through an HRA Event Tree

Appendix C Corrections to NUREG/CR-1278 and NUREG/CR-2254 C-1 Addendum \#1 to NUREG/CR-1278 $\quad \mathrm{C}-1$

Addendum \#1 to NUREG/CR-2254 C-5 
-vii-

LIST OF TABLES

page

1-1 Major Differences Between the ASEP HRA Procedure and THERP/Handbook 1-2

2-1 Definitions of Skill-Based, Rule-Based, and Knowledge-Based 2-6 Behavior

4-1 Procedure for Screening HRA of Pre-Accident Tasks 4-2

4-2 Basic and Optimum Conditions for Screening HRA of Pre-Accident 4-5

Tasks, Exclusive of Within-Person Dependence Effects

4-3 Applications of Table 4-2, Exclusive of Within-Person Dependence 4-7

Effects

5-1 Procedure for Nominal HRA of Pre-Accident Tasks

5-2 Basic and Optimum Conditions for Nominal HRA of Pre-Accident 5-10

Tasks, Exclusive of Within-Person Dependence Effects

5-3 Applications of Table 5-2, Exclusive of Within-Person Dependence 5-12 Effects

5-4 Guidelines for Assessing Within-Person Dependence Levels for 5-14 Nominal HRA of Pre-Accident Tasks

5-5 $\mathrm{F}_{\mathrm{T}} \mathrm{s}$ for Table 5-3 BHEPs, Modified for Multiple-Component Systems, 5-15

Assuming Dependence Levels Determined by Using Guidelines in Table 5-4, and Including RFs

6-1 Definitions of Cognition-Related Terms and Usage in the Handbook 6-4 of Human Reliability Analysis

7-1 Procedure for Screening HRA of Post-Accident Tasks 7-2

7-2 Initial-Screening Model of Estimated HEPs and EFs for Diagnosis 7-7 within Time $\mathrm{T}$ by Control Room Personnel of Abnormal Events

Annunciated Closely in Time

7-3 Assessment of Screening HEPs for Post-Accident 7-8

Post-Diagnosis Actions

8-1 Procedure for Nominal HRA of Post-Accident Tasks 8-3

8-2 Nominal Model of Estimated HEPs and EFs for Diagnosis within 8-10

Time $\mathrm{T}$ by Control Room Personnel of Abnormal Events Annunciated Closely in Time

8-3 Guidelines for Adjusting Nominal Diagnosis HEPs from Table 8-2 8-11

8-4 The Annunciator Response Model: Estimated HEPs for Multiple 8-12 Annunciators Alarming Closely in Time

8-5 Assessment of Nominal HEPs for Post-Accident $8-13$

Post-Diagnosis Actions

9-1 A Comparison of Some Individual and Total Failure HEPs Using 9-7

the ASEP HRA Procedure and THERP/Handbook

A-1 Correlation of Table and Figure Numbers Between ASEP HRA Procedure A-2 and RMIEP HRA Screening Rules

B-1 Explanation of Failure Limbs in Figure B-1 B-4

B-2 Sample Data File for Program UCBs-Propagation B-5

B-3 Program Output for Program UCBs-Propagation B-6

B-4 Computer Program for Program UCBs-Propagation B-7 
3-1 HRA Event Tree for EOMs for Hypothetical Tasks "A" and "B" 3-8

5-1 ASEP Mode1 for Assessing Within-Person Positive Dependence Leve1s 5-3 for Nominal HRA of Pre-Accident Tasks

6-1 Interaction of Cognition-Related Terms

6-2 Success and Failure Paths Following an Abnormal Event 6-7

6-3 Time Relationships between Annunciation of an Abnormal Event, 6-9 a Correct Diagnosis, and Performing the Required Post-Diagnosis Actions After a Correct Diagnosis

7-1 Initial-Screening Model of Estimated HEPs and UCBs for Diagnosis 7-6 Within Time $\mathrm{T}$ of One Abnormal Event by Control Room Personnel

7-2 HRA Event Tree for a Hypothetical Post-Accident Screening HRA

8-1 Nominal Model of Estimated HEPs and UCBs for Diagnosis within Time $\mathrm{T}$ of One Abnormal Event by Control Room Personnel

8-2 HRA Event Tree for a Hypothetical Post-Accident Nominal HRA

B-1 Expanded HRA Event Tree for Loss of Steam Generator Feed 


\section{ACKNOWLEDGMENTS}

Special credit is due Drs. Robert L. Brune and Meyer Weinstein, Human Performance Technologies, Inc., who (in addition to myself) provided the systems analysts on the Accident Sequence Evaluation Program (ASEP) with support in human reliability analysis (HRA), and to the four primary systems analysts who had overall responsibility for each of the four probabilistic risk assessments (PRAs) which included the HRA. The four systems analysts were: Robert Bertucio and David Moore, Energy Inc. (Kent, WA, office), who were the responsible systems analysts on the PRAs of, respectively, the Surry and Sequoyah pressurized water reactor (PWR) nuclear power plants, and Mary $\mathrm{T}$. Drouin and Alan M. Kolaczkowski, Science Applications International Corporation (Albuquerque Office), who were the responsible systems analysts for the PRAs of, respectively, the Grand Gulf and Peach Bottom boiling water reactor (BWR) nuclear power plants. All of the above people made several useful suggestions for improving the utility of the ASEP HRA Procedure, and the present version reflects these suggestions. In addition, Drouin and Kolaczkowski provided valuable information on nuclear power plant technical aspects related to an HRA.

A team of HRA specialists from Brookhaven National Laboratory, provided valuable task analysis information on certain accident sequences used in the PRA of the Peach Bottom BWR. These personnel were William J. Luckas, Jr., Dr. John N. $\mathrm{O}^{\prime}$ Brien, and $\mathrm{Dr}$. Richard L. Perline who performed an independent HRA for certain accident sequences for the Peach Bottom PRA (see Volume 4 of NUREG/CR-4550).

Special appreciation is due three staff members from the Reactor Systems Safety Analysis Division of Sandia National Laboratories. Gregory J. Kolb, initial project manager of ASEP, encouraged me to develop a shortened version of my HRA approach, and suggested some of the original screening values. Frederick T. Harper, the overall project manager of ASEP helped me understand some of the technical aspects necessary for the HRA, especially during trips to the Peachbottom and Grand Gulf BWRs and related training simulators to obtain relevant task analysis information on accident sequences of importance in the ASEP PRAs. Dr. Arthur C. Payne, Jr., served the same role on the Risk Methods Integration and Evaluation Program (RMIEP) and helped in the task analysis of pre-accident operations at the subject nuclear power plant, the LaSalle BWR. Dr. Payne also served as the systems analyst who applied a version of the ASEP HRA screening procedure to LaSalle BWR pre-accident tasks as part of RMIEP.

Finally, thanks are due to Elizabeth L. Frost, Statistics, Computing, and Human Factors Division, Sandia National Laboratories, who prepared the computer program for propagating uncertainty bounds in HRA Event Trees (in Appendix B), and to the following reviewers of drafts of the ASEP HRA Procedure: Donald K. Lorenzo, JBF Associates, Inc.; Professor Bjфrn Wahlstr $\phi \mathrm{m}$ and Pekka Pyy, Technical Research Centre of Finland; and Drs. Louise M. Weston and Dwight P. Miller, Statistics, Computing, and Human Factors Division, Sandia National Laboratories. The latter two reviewers made several suggestions to make the procedure easier to understand and to use. 


\section{LIST OF ABBREVIATIONS}

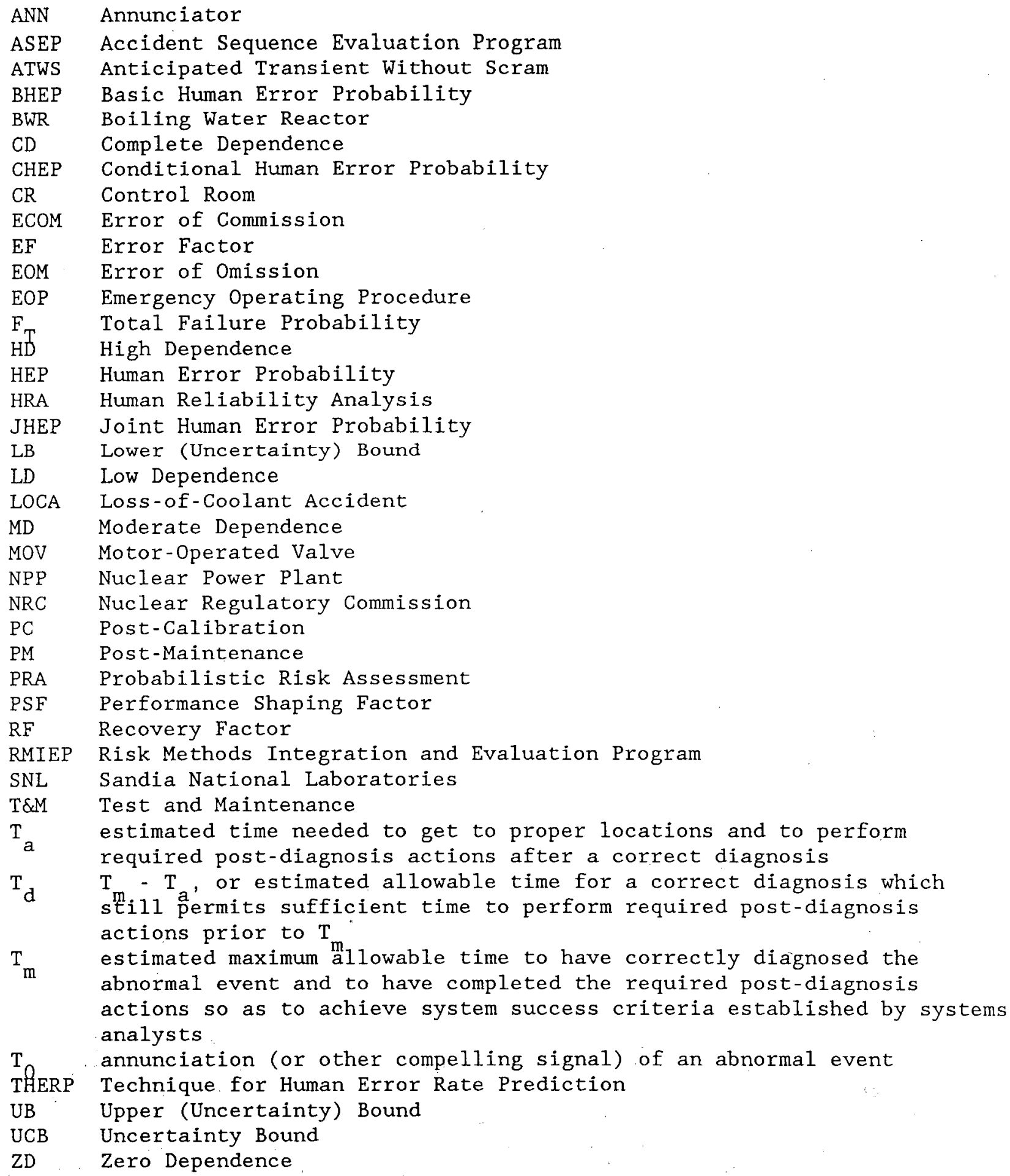


$-x i-$

DEFINITIONS OF TECHNICAL TERMS

Following are definitions of terms, many of which are taken from NUREG/CR-1278 (Aug. 1983), or which represent refinements of definitions of terms in that document. For purposes of the present document, some examples are given to clarify certain definitions. Any underlined term in a definition is also defined in this list of definitions. For definitions of other HRA-related terms, see the glossary in NUREG/CR-1278.

abnormal event (or condition or situation) - events that disrupt the normal conditions in a plant; in the context of this document, the occurrence of an initiating event, a loss-of-coolant accident, or system failures subsequent to the first two classes of abnormal events.

accident - an event or series of events in a plant resulting in an abnormal situation, requiring appropriate system response (including human response) to restore the plant to a safe condition.

action - carrying out of one or more activities (e.g., steps or tasks) indicated by diagnosis, operating rules, or written or memorized procedures.

activity - a general term referring to any kind of human performance, ranging from a simple motor action (e.g., flipping a toggle switch) to more complex behavior such as deciding which of two alternative courses of action to pursue. The complete sequence of activities in a pre-accident or postaccident condition include: perceive, discriminate, interpret, diagnose, decision-making, and action. Depending on the level of familiarity and skill involved, estimated human error probabilities for one or more of the intermediate activities between perceive and action may be assessed as negligible in a human reliability analysis.

administrative control - a general term referring to the kinds of checking of human performance mandated in a plant and the extent to which plant policies are carried out and monitored, including the use of tagging systems, suspense records, and associated inventory systems to ensure that safety-related systems or components are restored to their normal states after completion of maintenance, calibration, or testing.

annunciator (ANN) - a short term for an annunciated display, a legend indicator (or tile) with an auditory alarm to announce that a change of state has occurred.

anticipated transient without scram (ATWS) - a condition has occurred which requires an automatic trip of the reactor, but the automatic trip fails to occur. Failure of the backup manual trip by the operator is not included in the basic ATWS event.

arousal - see facilitative stress.

basic conditions - in the context of this document, basic conditions refer to the absence of recovery factors for human errors. Opposite of optimum conditions.

basic human error probability (BHEP) - the probability of a human error on a task that is considered as an isolated entity, i.e., not influenced by previous tasks.

between-person dependence - dependence of one person's behavior on the behavior of another. 
checker - one who is assigned to verify the accuracy of another's work, either while that person is doing the work or after its completion. The use of a checker is an example of human redundancy. A checker is not the same as the person who performs an inspection. The checker is "person oriented" whereas the inspector is "equipment oriented."

checklist - a written procedure in which each item is to be checked off with a pencil or other writing instrument as its status is verified.

coarse screening analysis - a screening analysis that employs very general screening rules, with little basis, if any, on a plant-specific task analysis, and which may result in unduly conservative estimates of human error probabilities and response times so that very little screening (i.e., elimination) of human events is done in the systems analysis. The opposite of fine screening analysis.

cognition - in the context of this document, restricted to those aspects of behavior involved in diagnosis of abnormal events.

common-cause failure - a failure which has the potential to fail more than one safety function and to possibly cause an initiating event or other abnormal event simultaneously, e.g., a human error that could result in miscalibration of several setpoints.

compelling signal - some kind of signal to the operator that is as demanding of attention as an annunciator.

complete dependence (CD) (dependence between two activities performed by the same person or between activities performed by different people) - a situation in which, if the relationship between the activities or people is positive (i.e., if there is positive dependence), failure to perform one activity correctly will result in certain failure to perform the other. Similarly, if success occurs in performing the first activity, success will occur on the other. The opposite results will occur if the relationship between the activities or people is negative (i.e., if there is negative dependence).

complete-failure path - the only path through an HRA event tree in which all activities are performed incorrectly. The usual application of the complete-failure path is for the case in which parallel systems or redundant components within a system can be defeated by an operator who fails to restore every one of these systems or components to its normal operating state after completion of calibration, maintenance, or testing of systems.

conditional human error probability (CHEP) - the probability of human error on a specific activity given failure, or success, on some other activity.

conditional human success probability - the complement of conditional human error probability.

conditional probability - the probability of an event occurring given that some other event has occurred.

conservative screening analysis - a screening analysis judged to be sufficiently conservative but not so conservative that the screening would eliminate only a few human error terms in the human reliability analysis from further consideration in the systems analysis. An ultra-conservative screening analysis may have the latter undesired result.

critical action. - in the context of this document, a critical action is one identified in the initial systems analysis as having the potential for putting some system or component at risk, e.g., the failure to restore an important blocking valve to its normally open condition following maintenance. 
-xiii-

critical parameters - in the context of this document, the critical variables pertaining to protection of the reactor core that control room operators are trained to monitor and initially respond to in the event of an initiating event, a loss-of-coolant accident, or other abnormal event. Typically, the immediate emergency actions that operators are required to memorize include the state of critical parameters.

decision-making - (1) decision-making as a part of diagnosis: the act of choosing among alternative diagnoses, e.g. to settle on the most probable cause of the pattern of stimuli associated with an abnormal event; (2) postdiagnosis decision-making: the act of choosing which actions to carry out after a diagnosis has been made; in most cases, these actions are prescribed by rules or procedures, and decision-making is not required.

dependence (between two activities) - the situation in which the probability of failure (or success) on one activity is different depending on whether a success or failure occurred on the other activity. The activities may be performed by the same person (within-person dependence) or by different persons (between-person dependence). For the same pair of activities, the level of dependence may differ for errors of commission and errors of omission.

diagnosis - the attribution of the most likely cause(s) of an abnormal event to the level required to identify those systems or components whose status can be changed to reduce or eliminate the problem; diagnosis includes interpretation and (when necessary) decision-making. This definition of diagnosis does not mean it is necessary to assign the proper name of the abnormal event in order to figure out what to do to cope with the event. The requirement for diagnosis in a post-accident situation can be minimized to the extent that the displays and emergency operating procedures clearly and unambiguously define the sequence of actions that are required after the initiation of some abnormal event.

discriminate - distinguishing one signal, or set of signals, from another, e.g., "the coolant level in Tank A is 37 feet," or, if there are limit marks on the meter, "the coolant leve1 is out of limits" (in the latter case, some interpretation is done for the operator by the design of the display).

disruptive stress - the bodily or mental tension resulting from the response to a stressor that threatens, frightens, worries, or angers a person, or increases that person's uncertainty, so that usually tasks are performed at a decreased level of effectiveness or efficiency.

doubling rule - when a person is required to take some corrective action in moderately to extremely high stress conditions with very limited time available to take the corrective action, if the first action is ineffective, his HEP for each succeeding corrective action doubles, up to the limit of an HEP of 1.0 .

dynamic task - one that requires a higher degree of interaction between the people and the equipment in a system than is required by routine, procedurally guided tasks. Dynamic tasks may include decision-making, keeping track of several functions, controlling several functions, or any combination of these. A post-accident task may be classified as a dynamic task if the written emergency operating procedure is so poorly written that it is difficult to follow with ease. The operator's tasks in coping with an abnormal event may be classified either as dynamic or step-by-step tasks. Pre-accident tasks are usually classified as step-by-step tasks, e.g., restoration of valves (to their normal operating states) after maintenance. 
emergency operating procedure (EOP) - special written procedures to assist operating personnel in responding to abnormal events. EOPs may be symptomoriented or event-based.

end-failure term - the probability of reaching the terminal point in a failure path through an HRA event tree. Contributes to total-failure term.

error - see human error.

error factor (EF) - the square root of the ratio of the upper to the lower uncertainty bound, the latter term as defined herein.

error of commission (ECOM) - incorrect performance of a system-required task or action, given that a task or action is attempted, or the performance of some extraneous task or action that is not required by the system and which has the potential for contributing to some system-defined failure.

error of omission (EOM) - failure to initiate performance of a system-required task or action.

event-based emergency operating procedure - emergency operating procedures keyed to events or systems associated with abnormal conditions rather than to the related symptoms or functions. Synonym: "system-oriented EOP." The intent of these EOPs is that the operator will diagnose the specific event causing the abnormal event or accident in order to mitigate the consequences of that situation. Opposite to symptom-oriented EOP.

event tree - a graphic representation of system events in which the events are designated by limbs in the tree, and the sequence moves forward in time. The event tree is an inductive mode1, whereas the fault tree is a deductive model. There are several forms of event trees; the event trees in this document (and in NUREG/CR-1278) are HRA event trees.

extraneous task or action - the performance of some activity not required by the system and which has the potential for contributing to some systemdefined failure. An extraneous action may occur despite an operator's correct diagnosis because he made a simple manipulation or selection error, or an extraneous action or series of such actions may occur because of an incorrect diagnosis.

extremely high stress level - a level of disruptive stress in which the performance of most people will deteriorate drastically. This is likely to occur when the onset of the stressor is sudden and the stressing situation persists for long periods. This level of high stress is associated with the feeling of threat to one's physical well-being or to one's self-esteem or professional status, and is considered to be qualitatively different from lesser degrees of high stress. The occasion of a large loss-ofcoolant accident is assessed as resulting in extremely high stress to operating personnel, as are some occasions in which more than two primary safety systems fail to function. Extremely high stress levels can be avoided by considerable practice on potential abnormal events so that the tasks can be classified as rule-based actions or skill-based actions. Synonym: "threat stress."

facilitative stress - the bodily or mental tension resulting from the internal response to a stressor that alerts a person, prods him or her to action, thrills a person, or makes him or her eager, so that usually the person performs at an optimal level of effectiveness or efficiency.

failure path - in the context of this document, any path through an HRA event tree that leads to an end-failure term. A failure path may have both success limbs and failure limbs. 
fault tree - a graphic representation of system events starting with some deviant condition and working backwards in time. The fault tree is a deductive model, whereas the event tree is an inductive model.

fine screening analysis - a screening analysis with the following primary characteristics: (1) it is based on an initial plant-specific task analysis, (2) it includes some credit for recovery factors for human errors, and (3) it takes into account certain possibilities of dependence among tasks which could result in common-cause failures resulting from within-person or between-person dependence. A fine level of screening analysis should provide more screening than a coarse screening analysis, but should still be capable of being judged to constitute a conservative screening analysis.

flow chart (or flow diagram) EOP - see graphics EOP.

function-oriented emergency operating procedure - see symptom-oriented emergency operating procedure.

general area - see: same general area

graphics EOP - an EOP which makes use of flow diagrams, usually binary in form, to show various paths of actions to follow in coping with an abnormal event. Synonyms: "flow chart EOP" and "flow diagram EOP."

high dependence (HD) - a level of dependence that is approximately midway between zero dependence and complete dependence on the continuum of positive dependence.

high stress - a level of stress higher than the optimum stress level, i.e., moderately high or extremely high stress level.

HRA event tree - an event tree representing a graphic form of task analysis in which the limbs designate human and other events as well as different conditions or influences upon these events. Success is designated by a left limb in a branching and failure is designated by a right limb. Small letters are used to label success limbs and capital letters are used to label failure limbs. The values assigned to all tree limbs (except those in the first branching) are conditional probabilities. The first limbs may also be conditional probabilities if they represent a carryover from some other tree. In any branching in the tree, the sum of the 1 imbs is 1.0 . The HRA event tree is drawn as a binary tree, i.e., only two limbs to each branching. Continuous variables are represented by one or more binary branchings. Synonyms: "probability tree diagram" and "THERP tree."

human error - any member of a set of human actions or activities that exceeds some limit of acceptability, i.e., an out-of-tolerance action where the limits of human performance are defined by the system. Synonym: "error."

human error probability (HEP) - the probability that an error will occur when a given task or activity is performed. The nominal HEPs in the tables in this document (and in NUREG/CR-1278) are judged to represent medians on lognormal distributions of HEPs. Synonyms: "human failure probability" and "task failure probability."

human failure probability - see human error probability.

human performance model - a qualitative or quantitative model that represents some aspect of estimated human performance.

human success probability (HSP) - the complement of human error probability, i.e., 1 - HEP. 
$-x v i-$

human redundancy - use of a person to check another's work or to duplicate the work. Synonym: checker. This term is the analog of equipment redundancy in a parallel system, i.e., at least two humans must err in order that human error contributes to the probability of some unwanted system condition.

human reliability - the probability of successful performance of the human activities necessary for either a reliable or an available system, specifically, the probability that a system-required human action, task, or $j$ ob will be completed successfully within a required time period, as well as the probability that no extraneous tasks or actions detrimental to system reliability or availability will be performed.

human reliability analysis (HRA) - a method by which human reliability is estimated. In this document, the HRA approach described in NUREG/CR-1278 is used, which is sometimes called "THERP/Handbook." See - Technique for Human Error Rate Prediction.

immediate emergency actions - those actions which must be taken quickly following an abnormal event, and which are supposed to be committed to memory by the operating personnel. See also skill-based actions.

independence (between two activities) - see zero dependence.

interdependence - some non-zero level of dependence.

initiating event - an abnormal event that requires the plant to trip.

inspection - the recovery factor when someone looks at items of equipment to ascertain their status. If the task is to check someone else's work, the job is designated as that of a checker. The inspector is "equipment oriented" whereas the checker is "person oriented."

interpret (interpretation) - the assignment of a meaning to the pattern of signals (or stimuli) that was discriminated, e.g., "the coolant level in Task $A$ is low, which means that the makeup pump is not running, or there is a leak somewhere, or the indicator is out of order"; if there is only one possible cause for the observed signal, the interpretation is equivalent to diagnosis.

joint human error probability (JHEP) - the probability of human error on all of the activities in the complete-failure path.

judgment - in the context of this document, this term is restricted to expert estimation.

knowledge-based actions (or behavior) - behavior that requires one to plan one's actions based on an analysis of the functional and physical properties of a system.

loss-of-coolant accident (LOCA) - a loss of reactor vessel coolant resulting from some defect such as a pipe break or leaky valve.

low dependence (LD) - a level of dependence that is greater than zero dependence but not very far up on the continuum of positive dependence.

lower (uncertainty) bound (LB) - the value of an uncertainty bound that is conservatively judged to correspond to the lower 5 th percentile of human exror probabilities on a lognormal scale of nominal HEPs.

man-machine system - a complex system consisting of people and equipment (including software and its programming and related displays)

man-machine interface - place and type of interaction between people in a system and hardware or software components, including people-to-people communication

misdiagnosis - an incorrect diagnosis of an abnormal event. 
model - an abstraction that represents symbolically the way in which a system functions operationally; generally, not all characteristics of a system will be included in a model. A model may be qualitative or quantitative.

moderate dependence (MD) optimum conditions - a level of positive dependence between low dependence and high dependence.

moderately high stress level - a level of disruptive stress that will result in a moderate deterioration in performance effectiveness of system-required behavior for most people. The onset of an abnormal event indicated by annunciators or other compelling signals is usually classified as resulting in at least a moderately high stress level. Synonym: "heavy task load."

negative dependence - the situation in which failure to correctly perform an activity reduces the probability of failure in performing another activity, or in which success in performing an activity reduces the probability of success in performing another activity In the usual HRA, negative dependence is seldom employed, as it would usually lead to optimistic estimates of HEPs. Instead zero dependence is usually employed.

nominal analysis - the regular probabilistic risk assessment in which the best (i.e., most accurate) estimates of failure probabilities are employed, as distinguished from the conservative (i.e., deliberately high) estimates used in a screening analysis.

nominal HEP - the probability of a human error in which the effects of plantspecific performance shaping factors have not been considered.

nominal HRA - a human reliability analysis in which nominal analysis is employed.

normal operating conditions - any plant operating conditions in a non-accident setting. Synonym: "pre-accident operating conditions."

operations personnel - personnel, usually licensed as well as unlicensed reactor operators, who are responsible for the daily operation of a plant.

optimum conditions - in the context of this document, optimum conditions refer to the presence of recovery factors for human errors. Opposite of basic conditions.

optimum stress level - the level of stress that is conducive to optimal performance. Most of the estimated human error probabilities in this document (and in NUREG/CR-1278) are predicated on the assumption of an optimum stress level, and must be adjusted upwards when non-optimum stress levels are assessed. Synonym: "optimum task load."

parallel system - in the context of this document, a parallel system is one in which the system fails only if all of the human actions in a set are performed incorrectly, and if at least one of the incorrect actions is not corrected by successful employment of a recovery factor. For example, in a system having two locally operated valves which are on redundant paths, an operator could cause some critical system safety function to be unavailable by forgetting to restore at least one of them to the normal state following completion of maintenance. Opposite of series system.

perceive (perception) - in the context of this document, used in the very narrow sense of "awareness" without the further meaning of "understanding," e.g., "some annunciator tiles over there are blinking"

performance shaping factor (PSF) - any factor that influences human behavior. PSFs may be external to the operator or may be part of his or her internal characteristics. 
-xviii-

plant policy - the operating requirements that plant management and supervision expect to be followed. Usually they are described in a formal set of written instructions that are available to all plant personnel. In some cases, they are not written but understood, e.g., the correct method of using a written checklist is to read one checklist item, perform the action required, and then read the next checklist item, and so on.

positive dependence - the situation in which failure to correctly perform a first activity increases the probability of failure in performing the second activity, and success in performing the first activity increases the probability of success in performing the second activity.

post-accident operating condition - a plant condition in which some abnormal event has occurred, and appropriate system responses (including human responses) are required to restore the plant to a safe condition.

post-accident task - all tasks required to cope with an abnormal event. Postaccident tasks are divided into diagnosis and post-diagnosis actions.

post-calibration test - a test to see that some component has been properly calibrated.

post-diagnosis actions - those actions that are to be carried out once a diagnosis of an abnormal event has been made.

post-maintenance test - a test to see that some component works properly after maintenance.

pre-accident operating conditions - see normal operating conditions.

pre-accident task - a term denoting activities done under normal operating conditions, including special conditions such as start up operations, or other activities that can affect the availability of equipment needed to cope with an abnormal event. Synonym: "test and maintenance task."

probability tree diagram - see HRA event tree.

recovery analysis - a term used by systems analysts to describe in probabilistic terms the ability of a system (including its operators) to "recover" from (i.e., cope successfully with) some abnormal event. Recovery analysis should not be confused with recovery factors.

recovery factor ( $R F$ ) - a factor that prevents or limits the undesirable consequences of a human error. One of the most common RFs is human redundancy. Other RFs are the effects on human performance of displays of component status in the control room (especially those which are annunciated), the effects of post-maintenance tests or post-calibration tests, and the effects of daily or shiftly inspections, especially those involving the use of written checklists.

response time - the time required to perform some critical action, including travel time.

restore or restoration task - the returning of valves, circuit breakers, and other components to their normal states after completion of maintenance, calibration, or testing. Restoration is not usually considered to be part of maintenance because operations personnel rather than maintenance personnel perform the restoration tasks. 
rule-based actions (or behavior) - behavior in which a person follows remembered or written rules, e.g., performance of written post-diagnosis actions or calibrating an instrument or using a checklist to restore manual valves to their normal operating status after maintenance. Rule-based tasks are usually classified as step-by-step tasks unless the operator has to continually divide his or her attention among several such tasks without specific written cues each time he or she should shift attention to a different task. In the latter case in which there is considerable reliance on memory, the overall combination may be classified as a dynamic task, especially in a post-accident condition.

same general area - in the context of this document, two components are in the same general area if they are no farther apart than a few steps. See same visual frame of reference.

same visual frame of reference - in the context of this document, two components are in the same visual frame of reference when they are in the same general area and the operator can see one of them without moving his or her head while performing some action on the other.

screening analysis - involves the use of conservative estimates of human behavior (i.e., higher human error probabilities and longer response times than one expects to be the case) to each system event or human task as an initial type of sensitivity analysis. If a screening failure probability does not have a material effect in the systems analysis, it may be dropped from further consideration.

screening HRA - a human reliability analysis in which screening analysis is employed.

sensitivity analysis - an analysis in which one or more estimates of various parameters are varied to observe their effects on a system or some part of it (e.g., in a human reliability analysis, estimates of human error probabilities would be varied to ascertain their effects in a systems analysis).

series system - in the context of this document, a system that will fail (or be designated as a failure) if any of the human activities in a set is performed incorrectly and not corrected by successful employment of a recovery factor. Opposite of parallel system.

skill-based actions (or behavior) - the performance of more or less subconscious routines governed by stored patterns of behavior, e.g., the performance of memorized immediate emergency actions following a loss-of-coolant accident or an initiating event, or the use of a hand tool by a person experienced with the tool. The distinction between skill-based actions and rule-based actions is often arbitrary, but is primarily in terms of the amount of conscious effort involved, in layman terms, the amount of "thinking" required.

skill-of-the-craft - a term describing those tasks in which it is assumed that workers know certain aspects of the job and need no written instructions, e.g., a plumber replacing a washer in a faucet. See skill-based actions.

step - an arbitrary division of a task or subtask that usually includes the following: some type of information presented to the operator, some degree of operator processing of the information, and some type of required response. A step may or may not be part of a detailed written procedure. 
step-by-step task - a routine, procedurally guided set of steps performed one step at a time without a requirement to divide ones attention between the task in question and other tasks. With high levels of skill and practice, a step-by-step task may be performed reliably without recourse to written procedures, e.g., repairing a faucet or the sequential performance of memorized immediate emergency actions. However, in such cases, the likelihood of errors of omission is increased. Pre-accident tasks or postaccident tasks may be classified as step-by-step tasks. See definitions for dynamic tasks, rule-based behavior, and skill-based actions.

stress - bodily or mental tension, ranging from a minimal state of arousal to a feeling of threat to one's well-being requiring action. Stress is the human response to a stressor. The effects of stress on human performance are curvilinear (i.e., non-monotonic), ranging from less than optimal performance when there are is a lack of sufficient arousal, through optimal performance with an optimum stress level, to extremely poor or disorganized performance at the extremely high stress level.

stressor - any external or internal forces that cause bodily or mental tension (i.e., stress).

subtask - a division of a task. The distinction between a task and a subtask is arbitrary, and used for convenience only.

suspense form or record - in the context of this document, written information describing equipment that is in a non-normal state because of maintenance, calibration, or tests, and when it is expected that the equipment will be ready for restoration to its normal state.

symptom-oriented emergency operating procedures - emergency operating procedures keyed to symptoms resulting from an abnormal event. Synonym: "function-oriented EOP." The intent of these EOPs is to enable the control room personnel to verify and maintain critical parameters without having to assign a name to the abnormal event in question. Symptom-oriented EOPs may be prepared as graphics EOPs, they may use columnar or narrative formats, or they may consist of some combination of any of these formats.

systems analysis - begins with the identification of initiating events, lossof-coolant accidents, or other abnormal events and the determination of the related accident sequences, which are the combinations of system successes and failures that lead to core melt following an abnormal event. The systems are analyzed, and the contribution to failure is determined and quantified to provide accident sequence frequencies.

system-oriented emergency operating procedures - see event-based emergency operating procedure.

tagging system - all those administrative controls that ensure (1) awareness of any valves or other items of equipment that are in a non-normal state and (2) prompt restoration of this equipment to the normal state after the completion of test, calibration, or maintenance operations. A tagging system includes the use of tags, chains, locks, and keys, and, in addition, logs, suspense forms or records, computer programs and printouts, and any other techniques that provide an inventory of the above items. 
talk-through - a task analysis method in which an operator describes the activities required in a task, explains what he or she is doing and the related mental processes during each activity in actual or simulated performance of a task. If the operator's performance is simulated, the operator merely touches the manual controls that would be operated in a real situation and describes the control manipulation required. The operator points to displays and states what readings would be expected, while describing any expected time delays and feedback signals and the implications to the plant function of operator activities. Synonym: "walk-through."

task - a level of job behavior that describes the performance of a meaningful job function; any unit of behavior that contributes to the accomplishment of some system goal or function. Usually a task is considered to consist of steps, and occasionally is broken down into subtasks.

task analysis - an analytical process for determining the specific behaviors required of the human components in a system. It involves determining the detailed performance required of people and equipment and the effects of environmental conditions, malfunctions, and other unexpected events on both. Within each task to be performed by people, behavioral steps are analyzed in terms of (1) the sensory signals and related perceptions, (2) information processing, decision-making, memory storage, and other mental processes, and (3) the required responses. The level of detail in a task analysis should match the requirements for the level of human reliability analysis of interest. A screening analysis requires considerably less task analysis than a nominal analysis.

task failure probability - see human error probability.

Technique for Human Error Rate Prediction (THERP) - a method for human reliability analysis to assess quantitatively the influence of human errors on the reliability or safety of a system. The method uses a schematic representation or abstraction of human events and related system events and their interactions (including levels of dependence). When conditional probability values are assigned to the limbs in HRA event trees used in THERP, mathematical estimates of the probabilities of achieving (or not achieving) certain combinations of events in the system may be obtained. THERP can accept data or estimates from any source.

test and maintenance task - see pre-accident task.

THERP tree - see HRA event tree.

total-failure term (or probability) $\left(\mathrm{F}_{\mathrm{T}}\right)$ - the sum of all the failure paths through an HRA event tree.

travel time - measured or estimated time to get from one location to another in the performance of system-required actions by operating personnel or their designates. Travel time is included in measures or estimates of response time.

ultra-conservative screening analysis - in the context of this document, a screening analysis which makes use of the upper uncertainty bounds of total-failure terms rather than the nominal $F_{T} s$.

uncertainty - as used in this document (and In NUREG/CR-1278), uncertainty includes random variability in some parameter or measurable quantity and an imprecision in the analyst's knowledge about models, their parameters, or their predictions. 
uncertainty bounds (UCBs) - the upper and lower bounds of human error probabilities that reflect the uncertainty in the estimation of an HEP. The UCBs include the variability of people and conditions and the uncertainty of the analyst in assigning HEPs to a task or activities in a task. The UCBs around the nominal HEPs in NUREG/CR-1278 are judged by the authors of that document to include the at least the middle 908 of the HEPs for that task. For conservatism, these UCBs may be assessed as representing the middle 908 range of the true nominal HEPs. (See definitions of lower bounds and upper bounds.) Uncertainty bounds are not the same as statistical confidence limits which are based on experiments.

upper (uncertainty) bound (UB) - the value in an uncertainty bound that is conservatively judged to correspond to the upper 95th percentile of human error probabilities on a lognormal distribution of nominal HEPs.

visual frame of reference (see: same visual frame of reference)

walk-through - see talk-through.

within-person dependence - dependence of the performance of one activity performed by a person upon the performance of another activity performed by the same person.

zero dependence (ZD) (between two activities) - the kind of dependence in which the probability of failure or success on one activity is the same regardless of whether failure or success occurred on the other. The activities may be performed by the same or different persons. Synonym: "independence." 
Accident Sequence Evaluation Program

Human Reliability Analysis Procedure

EXECUTIVE SUMMARY

The approach to human reliability analysis (HRA)* described in the August 1983 issue of NUREG/CR-1278, "Handbook of Human Reliability Analysis With Emphasis on Nuclear Power Plant Applications," has been used in several probabilistic risk assessments (PRAs) of nuclear power plants (NPPs) and other complex systems. The approach, sometimes called "THERP/Handbook," is based on a thorough task analysis of the human operations being assessed in a system. The task analysis includes the interfaces and interactions between people and equipment and among people in a system. A primary purpose of the task analysis is to assess fully the underlying performance shaping factors (PSFs) and dependence effects that impact the reliability of human performance.

Because the THERP/Handbook approach is thorough, for its fullest application it requires considerable manpower and time on the part of a team of experts, including a human reliability specialist, systems analysts, plant personnel, and others. The Nuclear Regulatory Commission (NRC) expressed a need for an HRA method that would provide estimates of human error probabilities (HEPs) and response times for tasks performed during normal operating conditions and post-accident operating conditions and that would be sufficiently accurate for PRA and yet require only a minimal expenditure of time and other resources. To meet this need, a new HRA approach was developed as part of the NRC's Accident Sequence Evaluation Program (ASEP), being managed by Sandia National Laboratories (SNL). This new approach is based heavily on the THERP/Handbook method for HRA, but incorporates many simplifications of the human performance models and HRA methodology in NUREG/CR-1278 to meet the above need. The first application of this new approach was made in the ASEP, in which four PRAs were performed in a short period of time. The new HRA method was a part of each PRA and much of the HRA was performed by systems analysts with a minimum of guidance from HRA specialists knowledgeable in the technology of human performance.

The new procedure, hereafter called the "ASEP HRA Procedure," is divided into procedures for pre-accident tasks and post-accident tasks. Pre-accident tasks considered are those which if performed incorrectly could result in the unavailability of necessary systems or components in a complex plant such as an NPP to respond appropriately to an accident. Post-accident tasks are those which are intended to assist the plant to cope successfully with an abnormal event, that is, to return the plant's systems to a safe condition. The ASEP HRA Procedure is further divided into procedures for screening HRAs and nominal HRAs. A nominal HRA is the HRA applied to those human tasks which survive the screening analysis which is part of the systems analysis. The screening analysis uses deliberately conservative (i.e., pessimistic) estimates of HEPs, response times, dependence levels, and other human perform-

*A1l technical terms are underlined the first time they appear in this Executive Summary and they are defined in the "Definitions of Technical Terms," the immediately preceding prefatory section. 
-xxiv-

ance characteristics, while the nominal HRA uses what the HRA team judges to be more realistic values, but still somewhat conservative to allow for the team's (any team's) inability to consider all possible sources of error and all possible behavioral interactions. Thus, the ASEP HRA Procedure consists of four procedures - - a Pre-Accident Screening HRA, a Post-Accident Screening HRA, a Pre-Accident Nominal HRA, and a Post-Accident Nominal HRA.

The basic approach taken in the development of the ASEP HRA Procedure was to select certain generic HEPs for certain sets of tasks and to employ easy-tounderstand procedures for use of these HEPs and for estimating the effects of dependence and recovery factors. This approach represents a major simplification in HRA, as compared with the THERP/Handbook approach in which many tabled entries must be used, and the HEPs from these tables modified by the effects of plant-specific PSFs, using other tables. The goal was a rule-based procedure, that is, one that could be employed with considerably less judgment than is the case with the more complete HRA procedure. To achieve this goal, and to avoid undue optimism in estimating the effects of human errors, several conservatisms were employed. The details and underlying rationale and assumptions of the basic approach, including the conservatisms, are described in the main body of this document. The following four paragraphs summarize some of the characteristics of each of the four HRA procedures.

The Pre-Accident Screening HRA emphasizes restoration errors and is based on the use of a .03 generic HEP as the basic human error probability (BHEP) for the combined effects of an error of omission (EOM) and error of commission (ECOM) for each task considered in the screening analysis. For screening, two primary criteria are used in deciding which tasks to consider: (1) tasks for which there is a potential for human errors to result in a common-cause failure, and (2) tasks which have no or only one recovery factor (RF) or only two RFs which involve human redundancy. Credit for RFs related to human interaction is severely limited. Zero dependence (ZD) is assessed for critical actions or tasks related to a series system, and complete dependence (CD) is assessed for critical tasks or actions related to a parallel system, except for those on parallel trains or components performed on different shifts, in which case $Z D$ is assessed.

The Pre-Accident Nominal HRA extends the screening HRA by allowing more credit for RFs and includes a rule-based methodology for assessing dependence. Provision is made for a reassessment of the BHEP of .03 on the basis of a more detailed analysis of the plant's administrative control procedures and their implementation. Application of the Pre-Accident Nominal HRA to one boiling water reactor was made by an independent HRA specialist (i.e., not the developer of the new procedure) who judged that the results were somewhat more conservative than results that would have been obtained using the THERP/Handbook HRA method that he has used in other applications. 
The Post-Accident Screening HRA uses the screening diagnosis model from NUREG/CR-1278 for estimates of diagnosis HEPs and response times, with special allowances for the practice of the recognition of deviations from critical parameters related to reactor/containment integrity. For estimates of screening HEPs for post-diagnosis actions, an HEP of 1.0 is assessed (1) for critical actions performed outside the control room, (2) for any critical actions for which there is no written procedure (even for actions to be memorized), and (3) for cases in which the required instrumentation fails or is misleading. Conservative assumptions are made about response times in the control room, and a generic HEP of .05 is assessed for all critical postdiagnosis tasks, except for memorized post-diagnosis immediate emergency actions constituting skill-based behavior, in which case a generic HEP of .01 is assigned.

The Post-Accident Nominal HRA employs the nominal diagnosis model (with its table for adjustments) from NUREG/CR-1278, with special allowances for the practice of the recognition of deviations from critical parameters related to reactor/containment integrity. Compared with the screening procedure, less conservative (presumably more realistic) HEPs and credit for more than one person are allowed for the post-diagnosis actions, and emphasis is placed on measurement (rather than estimation) of simulated response times. Application by the author of the post-accident nominal HRA procedure to some hypothesized Anticipated Transient Without Scram (ATWS) accident sequences in the PRAs for two boiling water reactors showed that the HRA results using the ASEP HRA Procedure were somewhat more conservative than those based on the standard HRA procedure and data tables described in NUREG/CR-1278. A second human reliability analyst reached the same conclusion in a similar analysis.

In addition to the above comparisons between the standard THERP/Handbook HRA approach and the ASEP HRA Procedure, another purpose in trying out the latter procedure was to ascertain to what extent it could be used by systems analysts with minimum support from human reliability analysts who have a detailed background in the technology of human performance. Due to time constraints, none of the ASEP PRAs used a screening HRA; only the nominal HRA procedures were employed. In the nominal HRAs performed for ASEP, three of the four systems analysts involved used the pre-accident nominal HRA and all four used the post-accident nominal HRA. Each analyst made suggestions for improving the utility of the ASEP HRA Procedure. Nearly all of their suggestions have been incorporated in this document. None of the four analysts believes that an HRA should be done without any support from a specialist in HRA; they did differ in details on the amount of support required. With the changes they suggested be made to the HRA procedure, the analysts stated they believed the procedure would be easy to apply, although, as discussed in the main body of this document, there were some reservations expressed about either too much conservatism, or, in fewer cases, not enough conservatism.

In addition to the above tryouts, the Risk Methods Integration and Evaluation Program (RMIEP), another NRC program managed by SNL, offered an additional tryout. In this program, an HRA pre-accident procedure developed by the author is almost identical to the ASEP HRA pre-accident nominal procedure. This RMIEP HRA procedure was employed by a systems analyst with a minimum of indoctrination from the author. The analyst experienced no problems in applying the procedure correctly, as evaluated by the author. 
The tryouts of the ASEP HRA Procedure demonstrated the feasibility and usefulness of an HRA approach that is not as detailed as the THERP/Handbook approach. The new method can be used by systems analysts with some guidance from specialists in HRA, especially in the difficult area of diagnosis errors, to ensure that undue optimism is avoided. The ASEP HRA Procedure should be regarded as a first-cut or approximate HRA, which will generally provide conservative estimates of the human contribution to pre-accident and postaccident unavailabilities or failures. The THERP/Handbook approach, when applied by a full-time team of systems analysts, a human reliability analyst, and appropriate plant personnel (specifically including one or more licensed senior reactor operators), will enable a more detailed and accurate systems assessment of the impact of potential human errors. It can be useful to apply the more detailed HRA procedure to those human/system interactions that are assessed as contributing materially to the systems analysis, based on the HEPs and response times obtained using the ASEP HRA procedure. 
PART I. OVERVIEW

This document provides a procedure for conducting a human reliability analysis (HRA) as a part of a probabilistic risk assessment (PRA) of a nuclear power plant (NPP). Prior to proceeding further in the document, it is suggested that the reader study the "Definitions of Terms" and "Executive Summary" in the prefatory pages.

This part consists of Chapter 1, "Introduction," and Chapter 2, "Concepts, Assumptions, and Limitations." Chapter 1 describes the purpose and background of the HRA procedure, and the scope and organization of this document. Chapter 2 presents some important qualifications which should be considered before using the HRA procedure in this document. 
CHAPTER 1. INTRODUCTION*

\author{
Purpose and Background of the ASEP HRA Procedure
}

This document presents a procedure for human reliability analysis (HRA) which was developed to provide support to the NRC's Accident Sequence Evaluation Program (ASEP). ASEP was intended to represent a different approach to a probabilistic risk assessment (PRA) in that an attempt was made to model only the important factors and events in a PRA. It was intended that the ASEP would take some shortcuts in PRA without being a "limited scope" PRA. Because traditional PRAs, as described in the "PRA Procedures Guide" (NUREG/CR-2300, 1983), are very expensive, it was hoped that the ASEP would result in a method for PRA which would be much more economical to use, and yet which would pinpoint the important sources of risk to the public from nuclear power plants (NPPs). The PRA procedure used in ASEP was developed on an as-going basis during the performance of PRAs on the following four NPPs: Surry Pressurized Water Reactor (PWR) Unit 1 (Bertucio et al, 1987a), Peach Bottom Boiling Water Reactor (BWR) Unit 2 (Kolaczkowski et a1, 1986), Sequoyah PWR Unit 1 (Bertucio et al, 1987b), and the Grand Gulf BWR Unit 1 (Drouin et a1, 1987). The above reports are all volumes in NUREG/CR-4550, which also includes a report on methodology (Harper et al, 1987a) and a summary report (Harper et a1, 1987b).

To support the PRAs being done as part of ASEP, a procedure for HRA was developed with the above goals in mind and used in the ASEP PRAs. The present document replaces two drafts of the "ASEP HRA Procedure" actually used in ASEP (Swain, 1985b and c). The ASEP HRA Procedure does include some shortcuts when compared to the procedure described in NUREG/CR-1278, the "Handbook of Human Reliability Analysis With Emphasis on Nuclear Power Plant Applications" (Swain and Guttmann, 1983). NUREG/CR-1278 uses an HRA procedure called Technique for Human Error Rate Prediction (THERP), which began development at Sandia National Laboratories (SNL) in 1961 and which has undergone continued development through its application to many complex man-machine systems, including NPPs, beginning with the first full-scale PRA, WASH-1400 (NUREG-75/014, 1975). NUREG/CR-1278 represents the most complete statement of this HRA procedure as of 1983, and, in addition, includes human performance models and tables of estimated human error probabilities (HEPs) which are used in an HRA. Starting with "THERP/Handbook," as this HRA approach is sometimes called, simplifications of some of the models, e.g., the dependence model, were made, and other shortcuts developed which were intended to provide a less manpower intensive, full-scope ASEP HRA Procedure at a cost of somewhat more conservative assessments of the impact of human errors in the systems analysis in a PRA. In addition, it was intended that the new procedure be capable of being used by systems analysts without the usual training and experience in the technology of human behavior, which is the usual background of persons who specialize in human reliability analysis. Table 1-1 lists the major differences between the ASEP HRA Procedure and THERP/Handbook.

\footnotetext{
*A11 technical terms are underlined the first time they appear in this chapter, and they are defined in "Definitions of Technical Terms" in the prefatory pages.
} 
Table 1-1 Major Differences Between the ASEP HRA Procedure and THERP/Handbook (p1/2)

\section{ASEP HRA Procedure}

1. Underlying human performance models not provided; for situations not addressed, must go to NUREG/CR-1278.

2. Detailed screening procedure for pre- and post-accident HRA.

3. Less time \& effort required but at cost of accuracy. Example: a basic HEP (BHEP) of .03 for all pre-accident tasks \& a BHEP of.1 for nearly all failures of recovery factors (RFs).

4. More conservative (i.e., pessimistic) estimates of HEPs, response times, dependence levels, RFs, and other human performance characteristics.

Examples from pre-accident HRA:

a. BHEP of .03 assumes an error of commission (ECOM) is always possible if no error of omission (EOM) is made.

b. HEP of . 1 for RF failure.

c. Not all RFs used; each RF used only once, \& no credit given for an $R F$ unless a written checkoff list is used.

d. Only 3 dependence levels used: $\mathrm{ZD}, \mathrm{HD}$, and $\mathrm{CD}$; only positive dependence considered.

5. Fewer inputs to systems analysts needed from HR analysts, but much review by latter required.

\section{THERP/Handbook}

1. Contains basic human performance models, and underlying procedure for task analysis and HRA event trees; provides background for addressing unusual accident sequences \& events.

2. No screening procedure for preaccident HRA; not sufficiently prescriptive for post-accident HRA.

3. More analysis required, resulting in greater accuracy.

Example: HEPs need to be estimated for all tasks and RFs.

4. More realistic estimates. a. Most BHEPs <.03; ECOMs considered only if plausible.

b. Many estimated RF HEPs <.1.

c. All identifiable RFs used.

d. Full treatment of dependence: all levels used, both positive and negative dependence considered.

5. Requires more active and continual participation by HR analyst for best results. 
Table 1-1 Major Differences Between the ASEP HRA Procedure and THERP/Handbook (p2/2)

\section{ASEP HRA Procedure}

6. Estimates effects of using symptom-oriented emergency operating procedures (EOPs).

7. No provision for considering specific misdiagnoses of abnormal events.

8. Provides explicit consideration of HEPs for memorized immediate emergency actions.

9. Provides additional specific guidance on assessment of stress levels for accident sequences.

10. Provides computer program for propagating uncertainty bounnds through an HRA event tree.

\section{THERP/Handbook}

6. Does not.

7. Can be considered, but not covered adequately.

8. Some, not much, guidance in text in Chapter 12.

9. Not as clearly stated.

10. Has the full procedure, but not the computer program.

Prior to development of the ASEP HRA Procedure, a procedure for a screening HRA was developed (Swain, 1985a) as part of the screening analysis for the NRC's Risk Methods Integration and Evaluation Program (RMIEP). This HRA screening procedure was necessary because NUREG/CR-1278 does not include a procedure for a screening HRA for pre-accident tasks, and the screening HRA procedure for post-accident tasks was considered to be incomplete. The RMIEP HRA screening procedure, together with NUREG/CR-1278, constituted the starting point for the ASEP HRA Procedure, including the screening HRA and the nominal HRA for both pre- and post-accident tasks. Chapter 2 includes additional information on the need and uses of a screening HRA.

\section{$\underline{\text { Scope }}$}

It is intended that this shortened version of THERP/Handbook be used to assist utilities in evaluating the role of operating personnel in currently operating plants, help enable designers of future plant's to avoid major human reliability problems, and provide a quantitative base for the assessment of human errors in NPP safety and productivity. Because the ASEP HRA Procedure does involve some shortcuts, certain precautions are relevant, as stated in Chapter 2. An advantage of the procedure is that it can be employed by systems analysts, with some direction and support by qualified human reliability specialists, and that it is less labor intensive than the full HRA approach described in NUREG/CR-1278. Because the ASEP HRA Procedure was designed to 
provide somewhat more conservative estimates of human performance than would be obtained using the full HRA approach, even the nominal HRA in this new procedure can be considered to represent a kind of sensitivity analysis. It could be worthwhile to use THERP/Handbook for a more detailed analys is of those human error terms developed by using the ASEP HRA Procedure which the systems analysis finds contribute materially to system failure terms of importance.

While this HRA procedure is specifically directed to NPPs, it can also be used, with minor modifications, for performing HRAs in other large process plants, e.g., chemical plants, oil refineries, offshore oil production, and other power-generating plants. The procedure is also specifically directed towards risk assessment, but the estimated HEPs and the procedure in general could also be used to assess producibility issues. For applications of the ASEP HRA Procedure to other areas than risk assessment of NPPs, it is recommended that an HRA specialist be a permanent member of the team of persons to use the procedure.

\section{Organization of the Report}

This report consists of prefatory material, four major parts, references, and two appendices. The detailed table of contents in the prefatory pages serves as an index. Following the table of contents and lists of tables and figures are the acknowledgments and a list of abbreviations. Next are several pages of definitions that are intended to provide a background to systems analysts and others in human behavior and human reliability terminology. The prefatory pages end with an executive summary.

Part I, "Overview," consists of this introductory chapter plus Chapter 2, "Concepts, Assumptions, and Limitations."

Part II, "ASEP HRA Procedure for Pre-Accident Tasks," consists of three chapters. Chapter 3, "General Information for HRA Procedure for Pre-Accident Tasks," provides assumptions and other statements that pertain primarily to activities performed by operations personnel, instrumentation and control personnel, and maintenance personnel under non-accident conditions which can affect the availability of safety systems needed for coping with an accident sequence. Chapter 4, "ASEP Screening HRA for Pre-Accident Tasks," presents the step-by-step procedure, models, and tables of estimated HEPs that one can use in performing the screening HRA for pre-accident tasks. Chapter 5, "ASEP Nominal HRA for Pre-Accident Tasks," presents the same kind of information for the nominal HRA.

Part III, "ASEP HRA Procedure for Post-Accident Tasks," also consists of three chapters that address the same issues as the chapters in Part II, except that the procedure is for human activities which take place after the onset of some abnormal event. The three chapters are: Chapter 6, "General Information for HRA Procedure for Post-Accident Tasks," Chapter 7, "ASEP Screening HRA for Post-Accident Tasks," and Chapter 8, "ASEP Nominal HRA for Post-Accident Tasks." 
Part IV, "Concluding Comments," consists of two chapters. Chapter 9, "Results of Tryouts of the ASEP HRA Procedure," describes experiences by five systems analysts and three human reliability analysts in using the new procedure in the four PRAs that were completed. Included are both positive comments and criticisms by these personnel. Chapter 10, "Conclusion," presents a capsule evaluation of the procedure, with suggestions for additional study.

Following the References, Appendix A provides a comparison of the ASEP HRA Procedure and the RMIEP HRA Screening Rules that served as the starting point for the former. Appendix $B$ includes a computer program for propagating uncertainty bounds around estimated HEPs through an HRA event tree. Finally, Appendix C lists corrections to the underlying document, NUREG/CR-1278, and to its companion document, NUREG/CR-2254.

How to Use This Report

First, it is imperative that the user understand the definitions of technical terms and the abbreviations found in the prefatory pages. Persons outside the human factors area may not be familiar with terms used in the human reliability area, and human factors specialists without experience in PRA may misinterpret terms related to PRA.

Second, the concepts, assumptions, and limitations in Chapter 2 should be kept in mind when using the ASEP HRA Procedure. Clearly the ASEP HRA Procedure is no substitute for THERP/Handbook if a full-scale HRA is to be performed. However, as indicated in Table 1-1, the ASEP HRA does remedy some gaps in THERP/Handbook and parts of it are useful for even a full-scale HRA. Certain parts of the present document refer to NUREG/CR-1278 for greater detail or explanations of some of the basic concepts. In addition, reference is made to other documents for information on task analysis and other background information. Apart from this type of basic information which someone familiar with HRA may already have, the ASEP HRA Procedure is a stand-alone document.

The detailed procedure itself is divided into two stand-alone parts: Part II, the ASEP Procedure for Pre-Accident Tasks, and Part III, the ASEP HRA Procedure for Post-Accident Tasks. If an analyst is interested only in one of these classes of tasks, he need not read the other. Also, within each of these two parts, the procedure is divided into two stand-alone chapters: a chapter on the screening HRA and a different chapter on the nominal HRA. If an analyst is interested in a nominal HRA only, he need not consult the chapter on screening. However, within each part, the "general information" chapter should be read (i.e., either the one on pre-accident HRA or the one on post-accident HRA).

If the PRA, in which the results of the HRA are to be incorporated, includes uncertainty bounds (UCBs), it will be necessary to propagate the UCBs as sociated with each of the estimated HEPs in a given HRA event tree to estimate the UCBs around the total failure probability, $F_{T}$, of the tree. Two approaches are suggested. The first is to use a Monte Carlo procedure. The second, an algebraic method based on assuming a lognormal distribution for 
HEPs, is described in Appendix A of NUREG/CR-1278. Appendix B in the present document includes a computer program for performing the calculations described in Appendix A of NUREG/CR-1278.

As indicated in Chapter 9, the best use of the ASEP HRA Procedure, or any HRA procedure, for that matter, will be made by a team in which the following specialities are represented: systems analysts, HRA specialists, and plant control room personnel and others as needed. Each of these specialists has valuable expertise to contribute to an HRA. Without each source of expertise, serious gaps and errors can occur, e.g., undue optimism or pessimism about human performance and error recovery factors, failure to take into account the kind and amount of practice on each abnormal event of interest in the PRA, failure to understand the differences between stated plant policy and procedures and what actually occurs in the plant,. lack of appreciation for the effects of ergonomically sound or unsound design of man-machine interfaces, including written procedures, and so on.

The ASEP HRA Procedure is based on a task analysis of the human operations of interest, as described in Chapter 4 of NUREG/CR-1278 and illustrated in Chapter 21 of that document and in NUREG/CR-2254. The level of task analysis to be performed will always represent some compromise between what the HRA specialist would like to do, and that for which there are sufficient time and funds. In the ASEP PRAs, none of the three HRA specialists was completely satisfied with the level of task analysis permitted by the time and funding limitations. The ASEP HRA Procedure assumes a less-than-ideal, but still adequate, level of task analysis. If absolutely necessary, the screening procedures in Chapters 4' (pre-accident screening HRA) and 7 (post-accident screening HRA) could be done without a plant visit if sufficient documentation is obtained from the plant. However, for the nominal HRA, visits to the plant and to its simulator training center are essential. A plant visit is also recommended prior to performing a screening HRA, or at least as a follow-up check after an initial screening HRA has been performed. Because a goal of ASEP was to reduce the time and effort for a PRA, only one plant and simulator visit was allowed for the HRA personnel, and the procedure below makes that assumption.

The plant/simulator visit procedure below was based on the premise that the draft ASEP HRA Procedure that was then being used (i.e., Swain, 1985b and c) would be sufficient to perform the HRA for the Grand Gulf PRA (Drouin et al, 1987). This was the procedure actually used (with exceptions noted in brackets []) in the latter PRA. Minor changes have been made to this procedure to make it compatible with the present document. 


\section{Plant/Simulator Visit Procedure for Obtaining Information Needed for ASEP HRAs}

The plant visit procedure for HRA purposes is based on (1) the general procedures found in NUREG/CR-2254 and in Chapter 4 of NUREG/CR-1278, (2) on the search scheme in Chapter 20 of NUREG/CR-1278, and (3) the procedures followed in the ASEP and RMIEP HRAs. A visit to the plant-specific training simulator should be arranged to round out the visit to the plant control room, to obtain information about the simulator training in post-accident events, and to obtain or take photos of the simulator control room.

The plant/simulator visit should be made by a team consisting of a minimum of one human factors specialist who is a practitioner of HRA and one systems analyst. The best HRA is one which combines both of these people's technology and expertise. Each can learn valuable information from the other. In addition, the expertise of the plant and simulator personnel to be tapped during the visit is vital for an HRA.

1. Prior to the plant visit, study the following material:
a. ASEP HRA Procedure in this document
b. Through the middle of $p 30$ of NUREG/CR-2254
c. Ch. 4 of NUREG/CR-1278
d. The search scheme in $\mathrm{Ch} .20$ of NUREG/CR-1278

2. If possible, in advance of the plant trip obtain samples of test and maintenance (T\&M) procedures and other relevant normal operating procedures, emergency operating procedures (EOPs), restoration procedures, etc. Judge the quality of these materials by use of the information in NUREG/CR-1369 Rev. 1 and NUREG/CR-2005 Rev. 1 (Brune and Weinstein, 1982 and 1983) and $C h .15$ of NUREG/CR-1278. This judgment is one input in determining how to adjust the estimated HEPs and any estimated response times. (For a nominal HRA, estimates of response times are to be made only as a last resort; take response time measures whenever possible.) It is especially important to note whether the new symptom-oriented EOPs are being used. [Note: For the Grand Gulf HRA, step 2 had to be done in connection with the plant visit.]

3. In advance of the plant trip arrange for talk-throughs of samples of normal operating procedures, restoration procedures, and post-accident tasks. If at all possible, arrange to observe a sample of the first two types of tasks as they are being done. Arrange for photo taking as an aid to the analyst's memory. Pictures of valves, labels, circuit breakers, etc. are useful, as well as line drawings of control room panels. Because of security regulations, photographs of control room panels may have to be taken at the simulator. 
4. Once at the plant, or at the utility headquarters, study the administrative manual and all other paperwork related to how the utility intends that pre-accident and post-accident tasks will be carried out, the provisions for detecting and correcting human errors (i.e., recovery factors such as surveillance and restoration procedures, including the tagging system and job aids used), schedule and type of training, especially that to be given in the simulator, tests to evaluate proficiency of personnel skills. This step describes how the utility intends that tasks should be carried out and all the administrative control employed to ensure that they are carried out as intended.

5. When interviewing utility or plant personnel, describe the purpose of the visit, emphasizing that the intent is not to evaluate people, but to identify performance shaping factors (PSFs) necessary to perform an HRA. The words "human reliability" often connote an intent to evaluate individual people, especially in view of the "Human Reliability Program" in the military services. Such a connotation must be disavowed. Greater support from the utility can be obtained by promising to discuss any observed human factors problems with utility personnel before reporting these to any outsiders. It may be possible for the utility to plan corrective action which would permit the assessment of lower HEPs that would be possible otherwise. Of course, any such assessment must clearly state the underlying assumptions for corrective action.

6. In general, when studying man-machine interfaces, record any major exceptions to the standard ergonomics practices presented in MIL-STD-1472C (1984) and NUREG-0700 (1981), and obtain or take backup photos if possible. This type of information can be useful in justifying any relatively high estimates of HEPs for specific pre-accident or post-accident tasks. This information is also necessary for the analysts to assess the applicability of the basic HEP of .03 for pre-accident tasks for the plant in particular, as discussed in Chapter 3 . The .03 basic BHEP is based on an assumption of at least average quality written instructions and restoration procedures and associated administrative control. If the quality of any of these is judged to be inferior, the basic BHEP of .03 should be adjusted upwards to .05 . Such an adjustment would then change most of the HEPs in the pre-accident HRA procedure.

7. If a sample of real tasks can be observed, make the observations without comment to reduce the possible biasing effect of the observations. Then proceed with the talk-throughs. Ideally, the systems analysts would have already selected all the critical actions they judge to be important. If, because of scheduling restrictions, this preparation is not possible, observe as many different kinds of simulated tasks as possible, with emphasis on restoration tasks. The talk-throughs should include the operator's demonstration of actual travel and hands-on touching of controls to be manipulated, etc. He should demonstrate the use of all the relevant procedures and checklists, especially restoration procedures and checklists for pre-accident tasks and EOPs for post-accident tasks. 
8. Evaluate all the paperwork, including type and handling of tags, associated with restoration procedures. One of the observations or results from the talk-throughs should be how the paperwork is implemented. What kind of tagging and untagging procedure is actually used to assure that critical items such as valves are properly restored to their required positions? In terms of administrative control, to what extent are plant policies likely to be followed, and to what extent are shortcuts likely to be taken? It is in the shortcuts that human redundancy could be reduced or human initiated common-cause failures introduced. (See Chapter 16 in NUREG/CR-1278 for additional guidance.)

9. At the training simulator, obtain the information necessary to use Table 8-3 ("Guidelines for Adjusting Nominal Diagnosis HEPs from Table 8-2"). This information should be obtained for all the abnormal events that the systems analyst believes will be included in the PRA. The information should be obtained through observation of simulator exercises, talkthroughs of others when actual observation is not possible, and interviews with simulator subjects and simulator training personnel. If at all possible, arrange with the simulator personnel to include unannounced accident sequences of interest to the PRA in sequences normally given in simulator training. Requalification exercises are more valuable to the HRA than exercises with uncertified reactor operators.

10. Plant-available color pictures of the control room panels should be obtained and evaluated for their usefulness to the HRA. In addition to the usual far-view photos, close-up photos should enable one to read the displays and labels, at least with a magnifying glass. If the available pictures are not adequate, take pictures in the actual control room if possible, or in the simulator, if not possible. Far views and closeup views can be helpful, but the number of closeup views can be reduced if line drawings can be obtained.

11. Using a stopwatch, take several time measurements for travel time to areas that are likely to be important in the systems analysis, for example, travel time from the control room to the diesel generator room. Use a fast walk as the basis for the time measures. Take into account any times required for passing through security doors. If station blackout or other disruption of the electricity to the security doors will be a consideration, include these procedures in the time measures. 
12. Determine the manning and task assignments for both pre- and post-accident tasks. For the pre-accident tasks, this will include the type of person to perform the tasks and the type of person, if any, to perform immediate checking of the tasks. In addition, determine what kinds of special signals in the control room are associated with what kinds of recovery of pre-accident errors, and to what extent shiftly or less frequent checks are done using written checklists. The manner in which checklists are used is an important input to correct employment of the ASEP pre-accident HRA procedure. For the post-accident tasks, determine the minimum manning for routine control room operations, and the requirements for others to be present at what times into an accident sequence. If there have been any plant exercises in which such times were recorded, evaluate the design of the exercises to see if these times can be used in the HRA. For example, if everyone concerned knew that a plant blackout exercise was scheduled for a certain day, the measured times could be presumed to materially underestimate real-world times.

13. Using the ASEP HRA Nominal HRA Procedure in Chapters 5 and 8 , collect the information required for using the basic conditions and optimum conditions in Table 5-2, the dependence level characteristics in Table 5-4, and the post-accident variables in Tables $8-1$ and $8-4$. To the extent that this information cannot be obtained, or to the extent that specific tasks to analyze cannot be identified by the systems analyst prior to the plant visit, the conservative default values and assumptions stated in the document should be employed.

14. Make arrangements for telephoning plant personnel for additional information subsequent to the plant visit. It is safe to assume that additional information will be required. 
CHAPTER 2. CONCEPTS, ASSUMPTIONS, AND LIMITATIONS*

The basic concepts and many of the assumptions and limitations pertinent to the ASEP HRA Procedure can be found in NUREG/CR-1278, upon which the present "shortened version of THERP/Handbook" is primarily based. The present chapter presents only some of the ideas behind the ASEP HRA Procedure. These ideas include concepts, assumptions, and limitations, all intermeshed, because separating them would be artificial.

\section{Usefulness of Human Reliability Analysis}

The first assumption, of course, is that sufficiently accurate quantitative estimates of human performance can be made for the purposes of probabilistic risk assessment (PRA). This is a premise on which human reliability analysis (HRA) is based. A limitation is that such prediction often has a sizeable amount of uncertainty. Some would say that often the extent of the uncertainty is unknown. In the absence of a large storehouse of error relative frequencies and response times for all kinds of human tasks, one has to acknowledge that a certain amount of healthy skepticism regarding quantitative estimates of human behavior is warranted. Furthermore, the lack of precision in predicting human behavior combined with the usual emphasis of PRA on complex systems having some degree of risk to the public supports the effort to err on the conservative side in estimating human error probabilities (HEPs) and response times. That is, although one's goal is to estimate as accurately as possible HEPs and response times, conservatism means that if one does make an error of estimation, the estimates of probabilities and times should be larger rather than smaller.

On the positive side, it is the usual case that very gross estimates are acceptable for many purposes of PRA. This is true both for the "behavior" of equipment as well as for the behavior of humans. HEP estimates that might be off by a factor of 10 in either direction are sometimes acceptable.

\section{HEPs and their UCBS}

As used in this document (and in NUREG/CR-1278), each estimated HEP is assumed to represent a median value on a log-normal distribution of HEPs. As discussed in Chapter 7 of NUREG/CR-1278, it is recognized that other distributions often occur, but for PRA work, it is convenient to assume a log-normal distribution, and within wide bounds, it does not matter too much whether the distribution is exactly lognormal.

*A11 technical terms are underlined the first time they appear in this chapter, and they are defined in "Definitions of Technical Terms" in the prefatory pages. 
In both documents, the upper bounds and lower bounds of HEPs reflect the estimated uncertainty in the estimation of an HEP. The uncertainty bounds (UCBS) include the variability of people and conditions and the uncertainty of the analyst in assigning HEPs to a task. The UCBs from NUREG/CR-1278 are conservatively judged by the authors of that document to include at least the middle 908 of the HEPs for that task. This uncertainty of individual HEPs is not necessarily the same as the uncertainty associated with an estimate of the long-run HEP, averaged across people and conditions. Also, these UCBs are not the same as statistical confidence limits which are based on data obtained from direct measurement of human performance on tasks, excluding subjective data. Additional explanation of these qualitative UCBs is found in Chapter 7 of NUREG/CR-1278. In the present document (and in NUREG/CR-1278), error factors (EFs) are sometimes used in place of UCBs in the tables of estimated HEPs. An $E F$ is the square root of the ratio of the upper to lower UCB, with the constraints that the upper bound of the UCB is less than 1.0 and that on a log-normal distribution the EFs are symmetrical around the estimated median HEP, unless the distribution is truncated by an upper bound of 1.0 . In Chapter 5, the EFs in some of the tables represent EFs for estimated total failure probabilities $\left(F_{T} s\right)$ that are based on several HEPs in an HRA event tree. Such EFs were calculated by the computer procedure described in Appendix B. This computer procedure is based on a method for propagating uncertainty bounds through an HRA event tree, as described in Appendix A of NUREG/CR-1278.

The listing of probabilities to several decimal places in some of the tables of HEPs is not meant to indicate great accuracy in the estimates, but merely to facilitate traceability in an HRA. When these HEPs are used in an HRA, considerable rounding should be employed for the final answer given to systems analysts. That is, the total failure probabilities $\left(\mathrm{F}_{\mathrm{T}} \mathrm{s}\right)$ for the HRA event trees that are entered into the system fault trees or system event trees are rounded appropriately to avoid the appearance of inappropriate exactitude.

\section{Starting Point for the ASEP HRA Procedure}

The procedure assumes that the critical actions related to the man-machine interfaces have been identified by a team of systems analysts, human reliability analysts, and others as needed. This identification is not a one-shot task, of course, but must be continually evaluated and re-evaluated, especially to ensure that potential common-cause failures due to human error have not been overlooked. The ASEP HRA Procedure does not include a procedure for identifying critical man-machine interfaces. This is primarily the responsibility of systems analysts, but should include inputs from human reliability analysts. 
Screening and Nominal HRAs

As noted in Chapter 1 , the ASEP HRA Procedure includes procedures for a screening HRA and a nominal HRA. A screening HRA involves the use of conservative estimates of human behavior (i.e., higher human error probabilities and longer response times than one expects to be the case) to each human task considered in a PRA. A nominal HRA involves the use of the analyst's best estimates of HEPs and response times.

In a screening HRA, the screening probabilities and response times are assigned to each human task as an initial type of sensitivity analysis. If a screening value does not have a material effect in the systems analysis, it may be dropped from further consideration. That is, it is not included in the subsequent nominal HRA. Screening reduces to a manageable level the amount of more detailed analyses to be performed in the nominal HRA. It is necessary to make a satisfactory balance between too little and too much screening. If the screening numbers are much too large, as is likely to be the case when a coarse screening analysis is employed, very few events and tasks will be "screened out," and the follow-on nominal HRA may be unmanageable in terms of the resources available to perform the HRA. On the other hand, if a fine screening analysis is employed, there is a risk that the screening probabilities will be so small (or screening response times so short) that potentially important events and tasks will be erroneously screened out, and dropped from further consideration in the subsequent detailed (i.e., nominal) analyses. The HRA screening procedure in this document presents an attempt to achieve a balance between too little and too much screening. An exception is the procedure for an ultra-conservative screening analysis developed for the screening HRA for pre-accident tasks (described in Chapter 4). This procedure was developed at the suggestion of peer reviewers of the RMIEP screening HRA procedure (Swain, 1985a), and carried over into the ASEP screening HRA procedure. However, the method was not used in any of the four ASEP PRAs. In fact, none of these PRAs employed any screening in the HRA; only the nominal HRA procedure was used because of limitations in time and funds.

As stated in Chapter 1, the RMIEP screening HRA procedure was used as the starting point for both the ASEP screening HRA procedure and ASEP nominal HRA procedure. Modifications were made to the RMIEP HRA screening procedure to permit a somewhat more conservative screening HRA for ASEP. Both the ASEP and the RMIEP HRA screening procedures represent "fine screening" as opposed to more "coarse screening." An example of the latter is on page A-8 of EPRI NP3583 (Hannaman and Spurgin, 1984), in which .001, .01, and .1 are suggested as screening HEPs for, respectively, skill-based, rule-based, and knowledge-based actions (as defined later in Table 2-1). In the RMIEP HRA, it was decided to employ a finer level of screening similar to that which was used in the Arkansas Nuclear One (ANO) Unit \#1 PRA (Kolb et al, 1982). Therefore, the ANO HRA screening rules were used as a starting point for the development of RMIEP HRA screening rules more specific to the LaSalle boiling water nuclear power plant, the plant being used as the vehicle for RMIEP. The basic idea behind fine screening as opposed to coarse screening is that (1) unduly conservative HEP estimates can be avoided by some, but not very much, additional human reliability analysis, and (2) a sound background for the subsequent nominal 
HRA is framed by a fine screening approach. The fine screening analysis is more likely to identify areas in which additional HRA is needed than a coarse screening analysis.

\section{Use of Sensitivity Analysis}

As noted in Chapter 1 , the procedure for screening HRA and for nominal HRA in this document is a step-by-step procedure which can be followed in a logical sequence. In keeping with ASEP philosophy, the procedure represents less than a full-scale HRA of the type described in NUREG/CR-1278, but it is intended that the procedure not overlook any important human errors and dependence effects that could materially degrade system safety. Thus, the procedure includes some shortcuts to a full HRA, but these shortcuts are designed to err on the conservative side. In this sense, even the nominal HRA procedure represents a type of screening. Since the ASEP nominal HRA procedure does incorporate some conservatism, it can be appropriate to perform sensitivity analyses to see how much lower estimated HEPs would have to be before the estimated impact of human errors would not have a material effect in the systems analysis. If, say, a factor of 5 reduction would make a material difference in some sequence of actions, it could be worthwhile to perform a more detailed HRA on the human actions in question. If a factor of 10 reduction would make a material difference, it still might be appropriate to perform a detailed HRA inasmuch as the ASEP nominal HRA procedure does not always include credit for all recovery factors.

\section{Recovery Factors}

It is necessary to emphasize the distinction between some systems analysts' use of the word "recovery" and the human reliability analyst's use of the term "recovery factors" (RFs). These ASEP systems analysts think of recovery in terms of the ability of the system (including its operators) to "recover" from some abnormal event. They use the term, recovery analysis, to show quantitatively the extent to which the system is expected to recover from such an event. In the HRA field, the term, recovery factors, is used in a different sense. It is defined in the "List of Technical Terms" as "factors that prevent or limit the undesirable consequences of a human error." One of the most common RFs evaluated in an HRA is human redundancy, the use of a person to check another person's work or to duplicate the work. Other RFs usually evaluated are the effects of displays of component status in the control room (especially those which are annunciated), the effects of post-maintenance tests or post-calibration tests, and the effects of daily checks or walkaround inspections, if those RFs include the use of written checklists. In the ASEP HRA procedure for pre-accident tasks, no RF credit is given for the use of written checklists unless users of the checklists have been instructed to check off each listed item of equipment looked at, once the prescribed check has been completed. 
Skill-Based, Rule-Based, and Knowledge-Based Behavior

In the late 1970s, Jens Rasmussen and his coworkers at Ris $\phi$ National Laboratory, Roskilde, Denmark, developed a task classification scheme to distinguish between different levels of mental complexity in the performance of tasks (Rasmussen, 1980, 1981b, 1982, Rasmussen and Lind, 1982, Goodstein, 1981). This scheme distinguishes between skill-based, rule-based, and knowledge-based behavior, as defined in depth in the three-page Table 2-1. For PRA purposes, most of the human behavior of interest in a pre-accident HRA falls into the categories of rule-based and skill-based behavior, with emphasis on the latter. A post-accident HRA deals with all three categories, especially rule-based and knowledge-based behavior.

In recent years, growing emphasis in PRAs has been in the area of knowledgebased behavior, especially possible diagnosis errors which could result in taking too much time to figure out what to do. The Rasmussen terminology therefore has appeared in several recent PRAs, and is included in the ASEP HRA Procedure. Although the distinctions among the three sets of behavior becomes blurred at times, these categories are still useful.

\section{Misdiagnosis}

The ASEP HRA Procedure cannot be used to estimate the probabilities of different modes of misdiagnosis. Misdiagnosis is treated merely as the failure to diagnose correctly, that is, the specific effects of possible erroneous diagnoses of any abnormal event are not evaluated, including any subsequent operator behavior based on an incorrect diagnosis. However, it is assumed conservatively that any failure to correctly diagnose an abnormal event within the allowable time (as discussed in Chapter 6) will result in a core damage accident. For many PRA purposes, this treatment is adequate. If this simplification is not acceptable, the misdiagnosis area can be treated separately, using NUREG/CR-1278 as a guide, plus a suggested new approach described in Swain and Weston (1987).

Additional concepts, assumptions, and limitations in the ASEP HRA Procedure are stated in the chapters in Parts II and III. 
Table 2-1 Definitions of Skill-Based, Rule-Based, and Knowledge-Based Behavior (p1/3)

(Copy of Table 2 from Swain and Weston, 1987)

Skill-Based Behavior: Rasmussen (1981b) notes that skill-based behavior consists of the performance of more or less stored patterns of behavior, e.g., manual control of fuel rod insertion and withdrawal, or operating a crane. One primary characteristic of skill-based behavior is that no interpretation of the meaning of a display is required; the display must be completely unambiguous with regard to the required action to take.

Goodstein (1981) states that skill-based behavior refers to "...highly trained sequences of 'automated' behavior typical for frequently encountered tasks. In process systems, this would occur in: (1) Control and steering tasks where the operator is part of the loop, and (2) Manipulative subroutines in connection with the use of tools and equipment for test, calibration, maintenance, adjustments. At this leve1, the external information should act as signals which define the space-time relations, deviations, variations, margins between the control object - be it the thumb and forefinger in a manipulative task and the environment."

In their development of an HRA methodology which relies heavily on Rasmussen's concepts of skill-, rule-, and knowledge-based behavior, Hannaman and Spurgin (1984) state, "In skill-based behavior there is a very close coupling between the sensory input and the response action. Skill-based behavior does not directly depend on the complexity of the task, but rather on the level of training and the degree of practice in performing the task. While different factors may influence the specific behavior of a particular individual, a group of highly trained operators would be expected to perform skill-based tasks expeditiously or even mechanistically with a minimum of mistakes. For rule- and knowledge-based behavior, the connection between sensory inputs and output actions is not as direct as in skill-based behavior."

As Rasmussen notes, the dividing line between skill-based and rule-based behavior is fuzzy at times. A key point is that what is rule-based for one person can be skill-based for the person who is at such a high state of practice that he can execute a sequence of actions based on a single stimulus (or pattern of stimuli). For example, we classify as skill-based behavior the performance of well-memorized and frequently rehearsed immediate emergency actions when a reactor trip occurs in a nuclear power plant. In layman terms, very little thinking is required - if this, then that. Rasmussen and Lind (1982) state that skill-based performance rolls along without the person's conscious attention, and he will be unable to describe how he controls and on what information he bases the performance. 
Table 2-1 Definitions of Skill-Based, Rule-Based, and Knowledge-Based Behavior (p2/3)

(Copy of Table 2 from Swain and Weston, 1987)

Rule-Based Behavior: Rasmussen (1981b) uses the term rule-based behavior to denote behavior that requires a more conscious effort (than is the case for skill-based behavior) in following stored (or written) rules, e.g., calibrating an instrument. "The activity at the rule-based level is to coordinate and control a sequence of skilled acts, the size and complexity of which depend on the level of skill in a particular situation - one single decision to go home for dinner may be enough for driving you there, if the ride is not disturbed" (Rasmussen and Lind, 1982).

Goodstein (1981) states that rule-based behavior is "...applicable for familiar but longer and more complex work situations where conscious control of a (stored or prescribed) sequence of tasks is required. Elements of the procedure can activate skill-based behavior but the rule is predominant and must be followed in order to achieve the relevant goal. Thus, rules are sequences of state-action-check tasks where success or failure is judged in terms of resultant system state. A direct perception of the actual physical object where possible gives reliable performance. However, in the control room, the operator must rely on the displayed information to serve as signs about the state of the process - both to identify the situation and to be able to associate to the appropriate set of rules as well as to check the progress of the execution of the rules. If this information is not suitably definitive but consists merely of conveniently perceivable signs which, with experience, seem to be adequate, then the operator can be trapped when situations arise for which the signs are not sufficient indicators of state."

Hannaman and Spurgin (1984) state, "Rule-based behavior is governed by a set of rules or associations, which are known and followed. A major difference between the rule-based and the skill-based behaviors stems from the degree of practice. If the rules are not well practiced, the human being has to consciously recall or check each rule to be followed. Under these conditions the human response is expected to be less timely and more prone to mistakes, since additional cognitive processes must be called upon. The potential for error results from problems with memory, the lack of willingness to check each step in a procedure, and failure to perform each and every step in the procedure in the proper sequence." Thus, they categorize the latter type of behavior as rule-based.

In our usage, an example of rule-based behavior would be the performance of most test and calibration procedures. The technician characteristically follows written statements. However, sometimes, the written steps are repeated so frequently that he relies on his memory, with a greater probability of errors of omission. Another example of rule-based behavior is the use of emergency operating procedures (EOPs), in which it is required that the EOP be read aloud by one operator who checks on the step-by-step performance of the person or persons performing the required actions. 
Table 2-1 Definitions of Skil1-Based, Rule-Based, and Knowledge-Based Behavior (p3/3)

(Copy of Table 2 from Swain and Weston, 1987)

Knowledge-Based Behavior: Rasmussen (1981b) applies the term knowledge-based behavior to cases in which the situation is, to some extent, unfamiliar--that is, where considerably more cognition is involved in one's deciding what to do. "The information process used by a person in a specific unfamiliar situation will depend very much on subjective knowledge and preferences and detailed circumstances for the task" (Rasmussen and Lind, 1982).

Goodstein (1981) states that knowledge-based behavior is "..necessary where skills or rules are either unavailable or inadequate so that conscious problem solving and planning are called for in order to meet the demands of the unfamiliar situation which has arisen. In this mode, information needs to be treated as symbols which can be directly utilized and manipulated within the particular system model and structure which form the reference frame for thinking about the system. Proper symbols will avoid the need for resourcedemanding mental transformations. Examples from everyday include playing cards with their suits and numbers, chessmen, algebraic equations. Finding suitable process symbols is a more complex problem since representations of physical variables and relations at various levels are required."

Hannaman and Spurgin (1984) state, "When symptoms are ambiguous or complex, the state of the plant is complicated by multiple failures or unusual events, or the instruments give only an indirect reading of the state of the plant, the operator has to rely on his knowledge, and his behavior is determined by more complex cognitive processes. Rasmussen calls this knowledge-based behavior. The performance of the human being in this type of behavior depends on his knowledge of the plant and his ability to use that knowledge. This type of behavior is expected to be more prone to mistakes or misjudgments and require more time for the appropriate action to be taken."

We employ the term, knowledge-based behavior, in situations in which personnel have to interpret, diagnose, or use some level of decision-making, as defined in Table 6-1 in Chapter 6. Implicit in the definitions of skill-, rule-, and knowledge-based behavior is the idea that error frequencies can be reduced by designs of work situations in which requirements for knowledge-based behavior can be modified to requirements for rule-based behavior. This is a central rationale for the new symptom-oriented EOPs. 
PART II. ASEP HRA PROCEDURE FOR PRE-ACCIDENT TASKS

This part consists of Chapter 3, "General Information for HRA Procedure for Pre-Accident Tasks," Chapter 4, "ASEP Screening HRA for Pre-Accident Tasks," and Chapter 5, "ASEP Nominal HRA for Pre-Accident Tasks." Chapter 3 provides assumptions and other statements that pertain primarily to activities performed by operations personnel, instrumentation and control personnel, and maintenance personnel under non-accident conditions which can affect the availability of safety systems needed for coping with an accident sequence. Chapter 4 presents the step-by-step procedure, models, and tables of estimated HEPs that one can use in performing the screening HRA for pre-accident tasks. Chapter 5 presents the same kind of information for the nominal HRA. 
This chapter provides general information for the screening human reliability analysis (HRA) and the nominal HRA for pre-accident tasks (also in some PRAs called test and maintenance (T\&M) tasks). To develop rules for the screening and nominal HRAs, modifications were made to the HRA screening procedure used in the Risk Methods Integration and Evaluation Program (RMIEP) (Swain, 1985a), which in turn was based primarily on modifications of NUREG/CR-1278. See Appendix A for more detail on the relationship of the RMIEP screening HRA to the ASEP HRA for pre-accident tasks. The rules for estimating human error probabilities (HEPs) and uncertainty bounds (UCBS) for screening HRAs and nominal HRAs for ASEP pre-accident tasks are found in, respectively, Chapters 4 and 5 .

To develop an HRA procedure for pre-accident tasks, a simplified model of human behavior for these tasks was devised. The model includes a generic human error probability (HEP) of .03 that can be used for all such tasks, plus rules to adjust this basic HEP for the effects of recovery factors (RFs) and dependence effects. For estimating dependence effects, a special dependence model was developed, based on a simplification of the dependence model in NUREG/CR-1278. This chapter provides a short background for these models. For a more detailed background, see Swain (1985a) or a section on "Screening Rules for the Human Reliability Analysis in the Risk Methods Integration and Evaluation Program" in Volume 5 of NUREG/CR-4832 (Payne et al, 1987).

\section{Definitions of Terms}

Before proceeding further, it is suggested that the reader consult the prefatory pages to review the list of abbreviations and definitions of the following terms, which pertain to pre-accident tasks. Some of these terms are also defined in the text which follows.

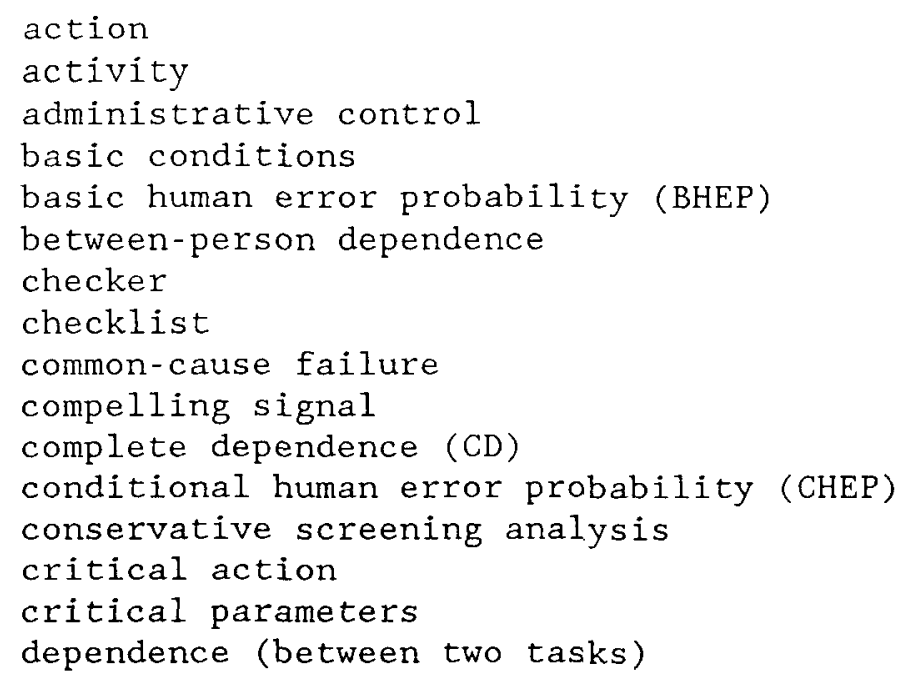




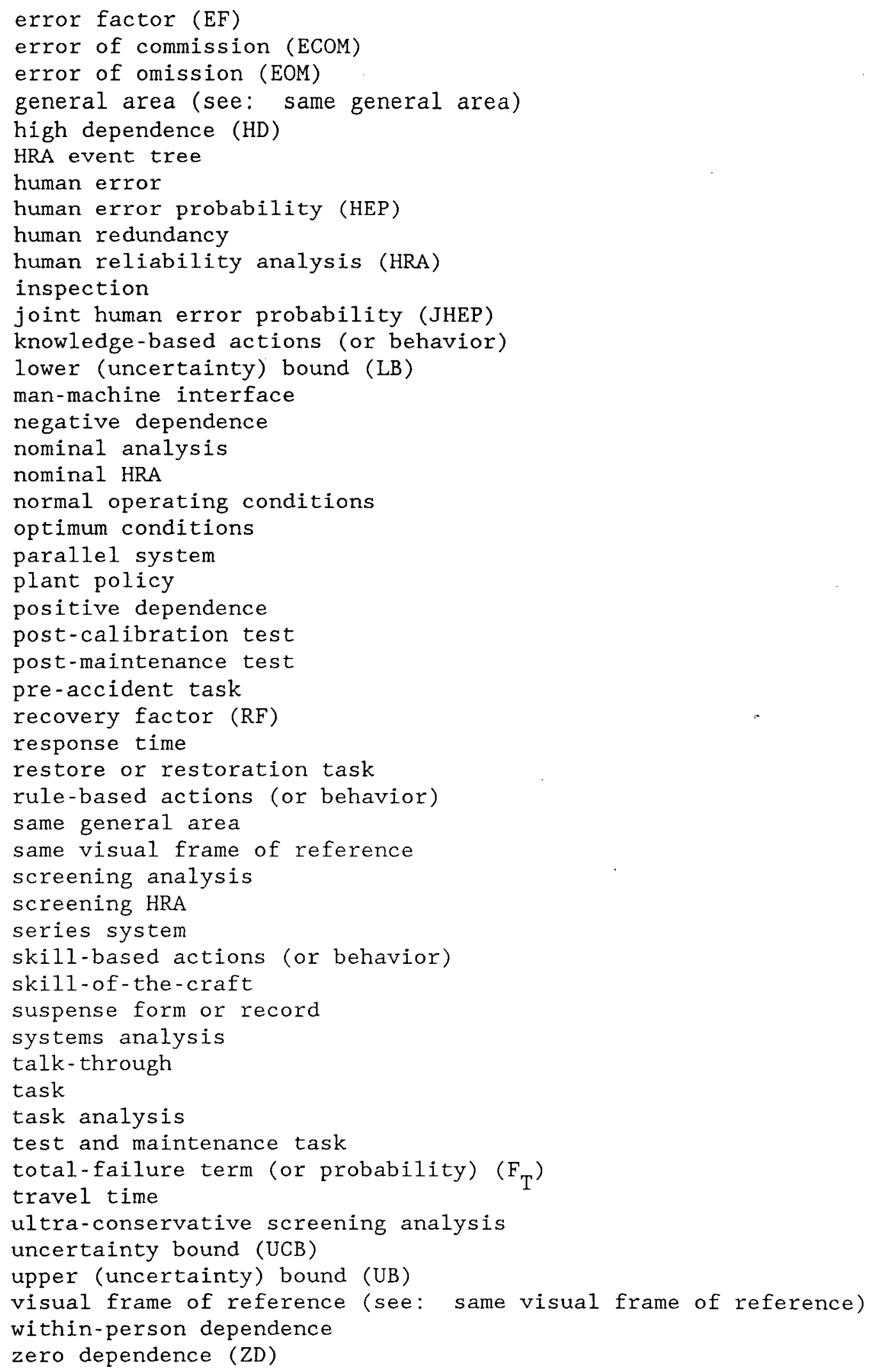




\section{Pre-Accident Tasks of Interest}

Pre-accident tasks typically of interest in a PRA consist of routine and corrective maintenance, calibration, surveillance tests, and restoration (i.e., the returning of components and systems to their normal conditions following maintenance, calibration, or testing). In some PRAs, the term "test and maintenance" (T\&M) is used as a synonym for pre-accident tasks.

In a typical PRA, some potential errors in the maintenance of components are not included in the systems analysis because such errors have already been counted in the failure rate estimates for components, and it would be inappropriate to count human errors twice. These kinds of maintenance errors usually refer to repairs or adjustments, e.g., repacking a locally-operated valve. In addition, other maintenance errors may not be counted in a PRA because of post-maintenance (PM) tests which, if done correctly, would discover such errors. Similarly, errors in most calibration tasks also are not included in the PRA because of post-calibration (PC) tests. It is imperative that the analyst identify those cases in which PM or PC tests, even if performed correctly, will not identify maintenance or calibration errors. The ASEP HRA procedure allows for this contingency as well as for the possibility that a PM or PC test is incorrectly performed.

In general, the tasks of primary interest in an HRA of pre-accident operations will be those in which restoration errors are possible. A typical restoration task consists of opening or closing of locally-operated valves (also called manual valves) or motor-operated valves (MOVs) after completion of some repairs or a test, so that these valves are restored to their normal status. (Some analysts include restoration tasks as "maintenance tasks," but other analysts do not because the plant personnel designated as maintenance personnel normally do not change the status of valves; this is done by operations personnel, usually auxiliary reactor operators.)

Although pre-accident tasks may include elements of skill-based, rule-based, or knowledge-based behavior as defined in Table 2-1, typically only rule-based behavior is of interest for PRA purposes when assessing pre-accident tasks. That is, the HRA considers how well persons carry out rules (usually, written rules). For the pre-accident HRA emphasizing the estimated quantitative impact of human performance on system safety, the interest is with potential errors only, and not with potential response time, i.e., how long it would take persons to perform the pre-accident tasks. For other HRA purposes, of course, response time could be of concern, as, for example, if an HRA is being used to compare the efficiency of different maintenance procedures in terms of manpower utilization.

As with the post-accident HRA procedure, the pre-accident HRA procedure begins with the assumption that the critical man-machine interfaces have been identified by a team of systems analysts and human reliability analysts. This identification is not a one-time endeavor, of course, but must be continually evaluated and re-evaluated, especially to ensure that potential common-cause failures due to human error have not been overlooked. 


\section{A Basic HEP for Pre-Accident Tasks}

A basic HEP of .03 was selected as a conservative HEP for pre-accident tasks as part of the developmental effort in the Risk Methods Integration and Evaluation Program (RMIEP). This basic HEP was also adopted for the ASEP HRA Procedure. The .03 basic HEP (BHEP) was based on some HRAs and additional reviews of pre-accident procedures at the LaSalle nuclear power plant (NPP), a boiling water reactor (BWR). These HRAs and some of the other developmental background are presented in Swain (1985a) or the section on "Screening Rules for the Human Reliability Analysis in the Risk Methods Integration and Evaluation Program" in Volume 5 of NUREG/CR-4832 (Payne et al, 1987).

The BHEP of .03 is for performance of pre-accident actions, exclusive of any recovery factors (RFs), and represents a combination of a generic HEP of .02 assessed for an error of omission (EOM) and a generic HEP of .01 assessed for an error of commission (ECOM), with the conservative assumption that an ECOM is always possible if an EOM does not occur. Thus for each critical action that must be accomplished, e.g., restore a valve to its normal operating position after maintenance, or perform a critical step in a calibration procedure, a total BHEP of .03 is used. The .03 represents the sum of the two possible failures, either (1) an EOM or (2) no EOM but an ECOM. Algebraically, the total-failure term (or probability) $\mathrm{F}_{\mathrm{T}}$ for a one-component system is,

$$
\mathrm{F}_{\mathrm{T}}=.02+(.98 \times .01)=.0298 \sim .03
$$

Reference to the tables in Chapter 20 of NUREG/CR-1278 will show that the .03 , and its constituents of .02 and .01 , represent conservatism in that these ASEP HEPs are larger than many of the basic HEPs in that document that are related to pre-accident actions.

\section{Recovery Factors for Pre-Accident HRA}

To assess the effects of recovery factors (RFs) on the BHEP of .03, a conservative approach was taken. First, each $R F$ is applied to the .03 rather than being applied separately for EOMs and ECOMs, a major conservatism. Second, the number of RFs that to be considered in the ASEP HRA Procedure is limited. For the screening HRA, the number of RFs to be considered is more severely limited than for the nominal HRA. Recovery factor HEPs are designated as .1, except for a $.01 \mathrm{HEP}$ of failing to perform or to perform correctly a required post-maintenance test or a post-calibration test. The 1 HEP is the nominal recovery BHEP for human redundancy stated as item \#1 in Table $20-22$ in NUREG/CR- 1278 .

The procedure makes a distinction between basic conditions in which no RFs are presumed to be available and optimum conditions in which allowable RFs are present. In the tables of RFs in Chapters 4 and 5 , each numbered basic condition has its same numbered complementary optimum condition. For a case in which all of the basic conditions apply, the BHEP of .03 is assessed as the human-caused failure of some critical safety component or system that is 
unavailable. For a case in which all of the optimum conditions apply, $F_{T}$ is considered to be negligible because of the multitude of RFs. For intermediate conditions, screening and nominal procedures are provided.

Some additional conservatisms are employed. For example, generally each available $R F$ is counted only once, even in the case in which one check is made at the site of the operation and a different one inside the control room. Similarly, even though there may be a requirement for a shiftly or daily check of component status, no credit for the RF is allowed unless a written checkoff list is required for the checker. For the screening HRA, this RF is counted only once. For the nominal HRA, at least initially the RF is counted only once. However, if the resultant joint HEP (i.e., the product of the BHEP and the RF HEP) turns out to have a material effect in the systems analysis, periodic checks may be counted more than once, using the procedure described in Chapter 9 "Unavailability" in NUREG/CR-1278. (For example applications, see NUREG/CR-2254.)

In addition to applying each RF to the BHEP of .03 rather than separately to each EOM and ECOM, the following conservatism is used. If there is more than one component to be checked in a group of components being treated as a "system" for analysis purposes, the relevant RFs are applied to the components as a group, rather than to each component individually. This means that each $R F$ is treated independently of the number of components in a system; each RF is counted only once to be conservative and, also, to account for the possibility that not all RFs will be employed on every occasion in which they should be employed. Thus, for any system, regardless of the number of components, the term at the end of each failure path in any HRA event tree can be multiplied by .1. This is equivalent to summing up the failure terms at the ends of all failure paths, without regard to RFs, and then multiplying the answer by the failure probability of an RF or the product of all failure probabilities of the RFs in question.

Dependence Effects for Pre-Accident HRA

The BHEP of .03 must be modified for the effects of dependence. The .1 HEP for failure of a recovery factor already includes the effects of betweenperson dependence between the person originally performing the task and the second person or other recovery factor. Therefore, what remains is to define rules for assessing the effects of within-person dependence, that is, dependence between the tasks performed by one person (in this case, the original task performer or the recovery factor performer).

The positive dependence model and other dependence guidelines in Chapter 10 "Dependence" in NUREG/CR-1278 require considerable judgment of a qualified HRA specialist for each set of tasks in which dependence must be assessed. For ASEP, it was necessary to develop a new dependence model and associated rules of application that could be used by systems analysts who do not have a formal background in human factors technology or HRA. 
In the ASEP treatment of dependence, dependence effects are treated differently for RFs and for original task performance. For RFs, dependence effects are not specifically considered because of the rule that in any group of tasks, each RF will be applied only once, and because even in the exceptions for periodic checks, independence can be assumed.

For original task performance, dependence effects are treated differently for parallel systems and for series systems. A parallel system is one in which $F_{T}$ occurs only if all components in a system are unavailable; system success occurs as long as at least one of the components is available. A series system is one in which system success occurs only if all components in a system are available; the failure of only one component renders the entire system unavailable, and is designated as $\mathrm{F}_{\mathrm{T}}$. Because of the large amount of component redundancy in nuclear power plants (NPPs), most applications of HRA are for parallel systems when more than one component defines the system.

For the screening HRA, dependence is treated very simply and conservatively. Zero dependence (ZD) is assessed among the same critical human actions on different components that are in series. For a parallel system, complete dependence (CD) is assessed among the same critical human actions on different components that are in parallel. However, if a testing schedule is such that different redundant trains or components within a train are tested or restored on different shifts, $Z D$ is assessed.

The treatment of dependence for the nominal HRA is more detailed. For parallel systems, zero dependence (ZD) is assumed for ECOMs while either ZD or some non-zero level of positive dependence can be assessed for EOMs. For conservatism, negative dependence is not used in parallel systems. For such systems, the use of positive dependence only results in conservatism.

For the treatment of series systems in the nominal HRA, the use of only positive dependence only results in a very small underestimation of HEPs, as long as the BHEP is not much larger than .01. The use of negative dependence would result in the most conservatism in a' series system, but would add a considerable amount of complexity in the judgments required in the assessment of dependence. Furthermore, for the usual BHEPs assessed in an HRA, the use of negative dependence would add only a very small amount of conservatism as compared to an assessment of zero dependence. Consequently, for the analysis of series systems, ZD is assessed for both ECOMs and EOMs. This seems to be a good balance between complexity avoidance and maximum conservatism. (Chapter 10 in NUREG/CR-1278 discusses positive and negative dependence in series and parallel systems and how to estimate their effects.)

Chapter 5 includes the detailed ASEP dependence model and procedure for the nominal HRA. 
An Example Illustrating Dependence Among Human Actions

Assume that there are some valves to be restored to their usual state after completion of some test or maintenance procedures. From a human factors viewpoint, the dependence among the restoration actions for these valves does not depend on whether the valves themselves are in series or in parallel with regard to how they work in the system. (We will assume that the operator's knowledge of whether they are in series or parallel has no major effect on his behavior.)

The operator may forget to initiate the entire restoration action because of distraction, or other influences. This error of omission (EOM) is not related at all to the design of the valves. In the dependence modeling for human actions, the probability of an operator making an EOM for the first valve to be restored is usually considered to include his probability of forgetting to initiate the entire restoration task. In this example we will drop this EOM from any treatment other than that associated with the first valve in the set.

Once the operator has initiated the task of restoration, he may forget any one or more of the valves in the set of valves (more EOMs), and for any valve he did not forget, he may make some error of commission (ECOM). For valve restoration, two kinds of ECOMs are possible that are usually considered in an HRA. One error would be the failure to fully open or fully close a valve. We can ignore this ECOM because the only credible way for this error to occur would be if the valve stuck in an intermediate position. Thus, the probability of this ECOM would be the joint probability of the valve's sticking and the operator's failure to note this equipment problem. This joint probability is usually assessed as being negligible. The other ECOM consists of a selection error. If any of the valves in a set are similar in position, appearance, or other factors, a selection error is likely. Usually, we consider that selection errors of this sort are completely independent, and we would assess a zero level of dependence (2D) among such errors. For purpose of this example only, let us ignore the effects of dependence on ECOMs and restrict the example to EOMs. (Remember, as noted earlier in this chapter, in the ASEP HRA Procedure for pre-accident tasks, it is always assumed that an ECOM is possible if an EOM is not made.)

NUREG/CR-1278 provides rules for assessing the dependence among the separate valve restoration actions with regard to EOMs. The dependence among the restoration actions can range from zero dependence (ZD) to complete dependence (CD). (We will ignore the possibility of negative dependence, and assume positive dependence only for this example.) If the valves are located in completely different rooms and are in different steps in a checklist, clearly there is little if any dependence among the actions. In such a case, we assess ZD. On the other hand, if the valves are all located in one central group, especially if they are all within the operator's visual field at once, we would usually assess CD for EOMs. For intermediate relationships, intermediate levels of dependence would be assessed.

Let us assume that we have only two different situations, the ones described above, and we have assigned $\mathrm{ZD}$ and $\mathrm{CD}$ to them as above. Let us simplify the problem and consider a set of valves to consist of only two valves, valves "A" 
and "B." Figure 3-1 shows an HRA event tree of this situation. The probability of an EOM for valve "A" is designated as the capital letter $A$. The probability of no EOM (i.e., 1 - A) is designated as the small letter a (the operator did remember to restore valve "A"). In HRA event trees, failure limbs are always indicated with capital letters, and are branched to the reader's right. Success limbs are indicated with small letters and are branched to the left. Because the HRA event tree is a binary decision tree, the probabilities assessed to the two limbs in each branching must sum to 1.0, and the sum of the probabilities at the end of all the success and failure paths through the tree also must sum to 1.0. Equivalent notation is used for valve "B," except for illustrative purposes that the appropriate conditionality has been conventionally indicated with the I sign, e.g., B/a means the probability of an EOM on Valve "B," given that no EOM has been made on valve "A." For simplicity, the full statement, B|a, is usually stated simply as B; the conditionality is understood. To avoid confusion in the B statements, B|A might be stated as B'(i.e., B prime). In the example below, the full statements are used. This standard notation is used throughout the report, and is described fully in Chapter 5 of NUREG/CR-1278.
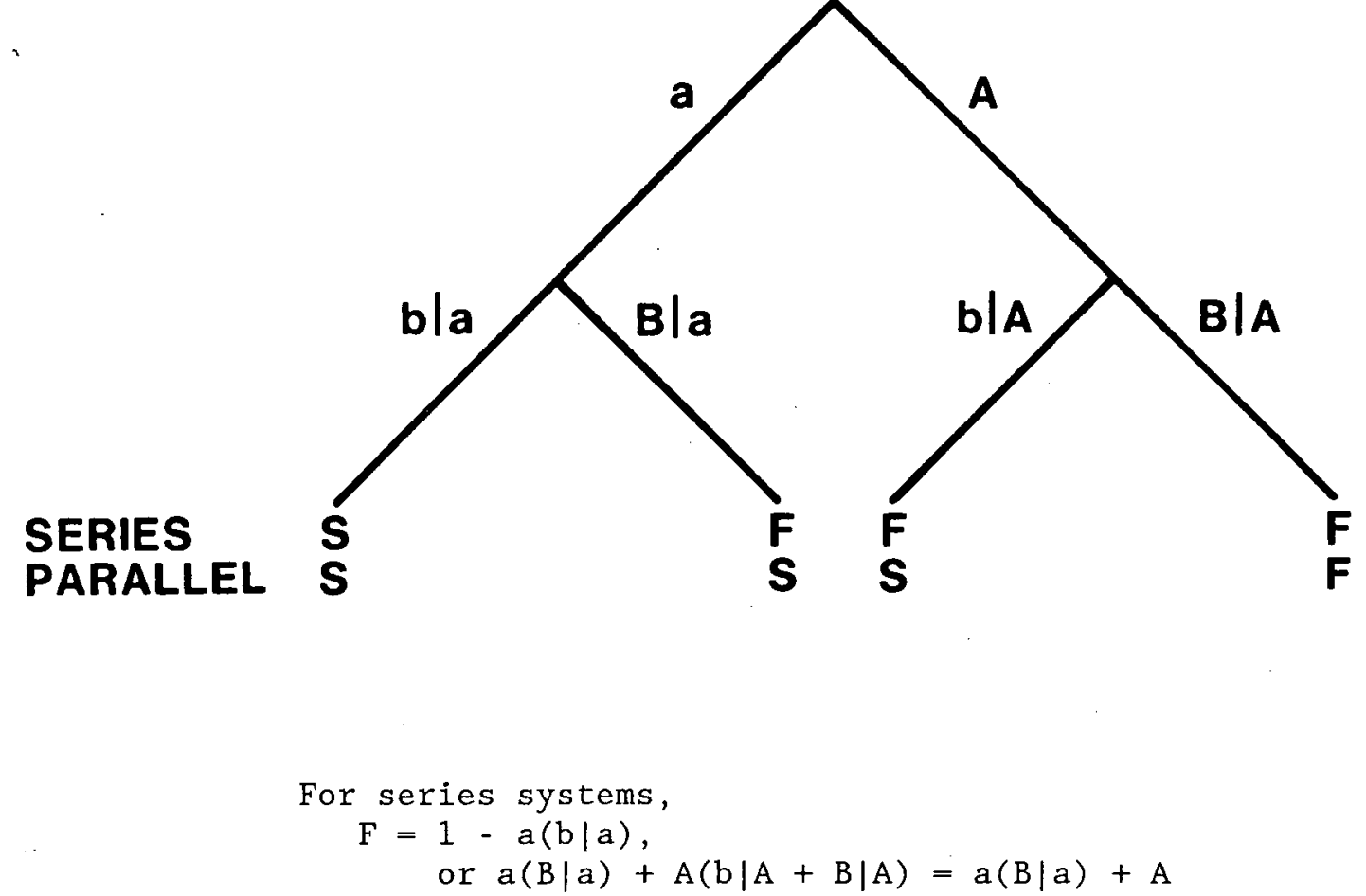

For parallel systems,

$$
\begin{aligned}
F= & A(B \mid A), \\
& \text { or } 1-[a(b \mid a)+a(B \mid a)+A(b \mid A)]
\end{aligned}
$$

Figure 3-1 HRA Event Tree for EOMs for Hypothetical Tasks "A" and "B" 
Consider the $Z D$ case first. The valves themselves might be connected in series (which we will call $2 D$, ser) or in parallel (ZD,par). If the valves are connected in series, then from a systems point of view, $F \mid Z D$, ser $=a(B \mid a)+A$, or 1 - $a(b \mid a)$. That is, if an EOM is made for either valve, the system fails; both valves must be restored for system success to occur, i.e., $S=a(b \mid a)$. If the valves are connected in parallel, then from a systems point of view, $\mathrm{F} \mid \mathrm{ZD}$, par $=\mathrm{A}(\mathrm{B} \mid \mathrm{A})$. That is, the system will fail only if both valves are not restored. If either is restored, system success will occur.

Now consider the $C D$ case. Again, the valves themselves might be connected in series (which we will call $C D$, ser) or in parallel (CD,par). If the valves are connected in series, then from a systems point of view, $F \mid C D$, ser $=a(B \mid a)+A$, or $1-a(b \mid a)$. If the valves are connected in parallel, then from a systems point of view, $F \mid C D, p a r=A(B \mid A)$. Note that the two sets of equations for $2 D$ and $C D$ are identical. The answers will be different when one puts in the appropriate conditional probabilities for the letter symbols denoting correct or incorrect human actions.

Let us assume in the case of the $Z D$ level of dependence that $A=B \mid A$. This equality is usually the case. It means that the basic human error probabilities (BHEPs) (i.e., the probabilities without considering the effects of dependence) of the two tasks ("A" and "B") are equal. This is what one would expect if the restoration task is to restore similar-appearing valves. For the $C D$ case, by definition $B / A$ must equal 1.0 and $B / a$ must equal 0 .

Assume that $A=B \mid A=.03$, that is, the BHEPs are equal to .03 . Now we can work out the system failure probabilities for the above four cases:

$$
\begin{aligned}
& F \mid Z D, \text { ser }=1-a(b \mid a)=1-.97^{2}=.0591 \sim .06 \\
& F \mid Z D, p a r=A(B \mid A)=.03^{2}=.0009 \sim .001 \\
& F \mid C D, \text { ser }=1-a(b \mid a)=1-(.97 \times 1.0)=.03 \\
& F \mid C D, \operatorname{par}=A(B \mid A)=.03 \times 1.0=.03
\end{aligned}
$$

The above simplified HRA problem illustrates the point that what is designated as system success is defined by the way in which the system works, and that assessment of levels of dependence among human actions is based only on the interrelationships anong the human actions themselves. The example also illustrates that for the typical HEPs used in an HRA of pre-accident tasks (i.e., HEPs not much larger than .01), dependence is an important consideration in the analysis of parallel systems, but not for series systems. 
CHAPTER 4. ASEP SCREENING HRA FOR PRE-ACCIDENT TASKS

\section{Genera1 Information}

In Chapter 2, the distinction between a nominal and a screening HRA was discussed, and also the distinction between a coarse and a fine screening HRA, as applied to pre-accident tasks. The ASEP HRA screening rules can be classified as a very fine level of screening. The primary characteristics of a very fine level of screening are (1) it is based on an initial plant-specific task analysis, (2) it includes some credit for human error recovery factors (RFs), and (3) it takes into account certain possibilities of task dependence that could result in common-cause failures resulting from within-person or betweenperson dependence. Note that in the ASEP screening HRA for pre-accident tasks each RF may be counted only once.

Either of two sets of estimated probabilities can be employed in the HRA screening procedure for pre-accident tasks. The first set involves the use of conservative total-failure terms $\left(F_{T} s\right)$ that have been calculated as described in Swain (1985a) and in the section on "Screening Rules for the Human Reliability Analysis in the Risk Methods Integration and Evaluation Program" in Volume 5 of NUREG/CR-4832 (Payne et al, 1987). The second set involves the use of the upper bounds (UBs) of the estimated uncertainty bounds on the $F_{\mathrm{T}} \mathrm{s}$, calculated by the uncertainty bounds propagation computer program described in Appendix $B$. The use of the UBs of the $F_{T} s$ provides an ultra-conservative screening procedure. It is important to emphasize that this ultra-conservative screening method uses the UBs of the $F_{T} s$; it does not involve the use of the UBs for every estimated HEP that went into the calculations of each of the $\mathrm{F}_{\mathrm{T}} \mathrm{s}$ in the above tables. Such an approach would be an "extreme worst-case analysis," and would amount to no screening at all.

Because of time and fund limitations, neither screening procedure was used in the ASEP PRAs. Both are included here for completeness.

\section{The Procedure}

Table 4-1 presents the steps to follow in applying the ASEP HRA Screening Procedure for pre-accident tasks, and Tables 4-2 and 4-3 present the tables of estimated HEPs and other rules for the HRA screening procedure for pre-accident tasks. For background material used to develop these tables, including the rationale for the basic HEP of .03, see Swain (1985a) or the section on the HRA screening procedure in Volume 5 of NUREG/CR-4832 (Payne et al, 1987). 
Table 4-1 Procedure for Screening HRA of Pre-Accident Tasks ( $\mathrm{pl} / 3$ )

1. Visit the plant initially to observe a sample of pre-accident tasks (especially calibration tasks, post-calibration and post-maintenance tests, and restoration tasks) and obtain relevant written procedures and other documentation that spells out operating sequences and rules.

a. The observations should include talk-throughs of several pre-accident procedures, emphasizing restoration tasks, and, if possible, some actual calibration tasks and post-calibration and post-maintenance tests. Failure to make these observations, including interviews with operating personnel who actually perform the tasks, will mean that the estimated HEPs will probably have to be higher than otherwise.

b. Carefully evaluate the quality of the administrative control, e.g., how well prescribed pre-accident tasks, especially human error recovery factors (RFs), will be performed. (See Chapter 16 in NUREG/CR-1278 for more detail.)

c. Other plant visits should not be necessary during the screening process, but may be necessary for the subsequent nominal HRA.

2. Based on the information obtained in step 1, determine if the basic HEP of .03 should be adjusted upwards for unusually poor human factors, especially written procedures. No downward adjustment is permitted.

a. As the .03 already is a conservative HEP, the decision for an upward adjustment should be fully documented. The human factors in a plant would have to be unusually poor to require an upward adjustment.

b. If it is not possible to make this evaluation because of inability to observe pre-accident tasks (or to receive talk-throughs of these tasks) or because of inability to evaluate adequately. the administrative control procedures, employ a .05 basic HEP in place of the .03 HEP. All the other numbers in the tables which are based on the .03 HEP will have to be changed, using the arithmetic implied by the tables.

3. Identify the pre-accident critical actions in terms of the systems analysis. (Note: Ideally, this step should be performed prior to the plant visit. In ASEP, this was not possible due to scheduling restrictions:) Obtain any additional written materials required.

4. Identify the recovery factors (RFs) listed below. Do not consider any other RFs. For each critical action, count each relevant RF only once.

a. Determine for which critical pre-accident actions, errors can be assessed as fully recoverable by "compelling signals, " usually one or more annunciators when a maintenance or calibration task is completed or before normal power operation can be resumed. 
Table 4-1 Procedure for Screening HRA of Pre-Accident Tasks(p2/3)

b. Determine for which critical pre-accident actions, errors will be recovered by a post-maintenance (PM) or post-calibration ( $P C$ ) test if the test is performed correctly. (Just because a test is scheduled does not guarantee that it will be performed, and performed correctly.)

c. Determine for which critical pre-accident actions, (1) a second person is required to directly verify component status after completion of the actions by the original performer, or (2) the original performer is required to make a separate check of component status at a different time and place from his original performance. An example of the latter would be for an operator to restore a locally-operated valve to its normal operating condition following maintenance, and then to return to the control room and note that an appropriate indicator light shows the desired position of the valve. No recovery credit is given for either check unless a written checkoff list is used during the check.

d. Determine for which critical pre-accident actions there is a requirement for a shiftly or daily check of component status in or outside of the control room, using a written list. No recovery credit is given for either check unless a written checkoff list is used during the check.

5. Consider all of the following critical tasks, i.e., include them in the screening analysis. (Exclude all other tasks.)

a. All tasks which have no RFs, as defined above.

b. All tasks for which there is a common-cause situation, i.e., incorrect eperformance of the task could fail redundant systems or components.

c. All tasks for which there is no common-cause situation and if any of the following RFs apply:

1) 4.b only

2) 4.c only

3) 4.d only

4) 4.c and 4.d in combination

6. Assign a basic HEP (BHEP) of .02 for each error of omission (EOM) and .01 for each error of commission (ECOM). Assume that an ECOM is always possible if an EOM is not made. Therefore, for each critical action, assign a total BHEP of .03.

7. Assign a .1 HEP for failure of each relevant $R F$, except for a .01 failure to perform a required $\mathrm{PM}$ or $\mathrm{PC}$ test or to perform it correctly. The RF already includes between-person dependence, and is assumed to apply to the EOM and ECOM as a unit, i.e., to the BHEP of .03 for a complete critical action. Therefore, it is appropriate to multiply the .03 by the 
Table 4-1 Procedure for Screening HRA of Pre-Accident Tasks (p3/3)

product of the RF HEPs and to treat the final product as a unit. It is judged that the assessed probability of failure of each RF is high enough to also include estimated failures of administrative control, i.e., the failure to perform the prescribed RF. (See Chapter 16 in NUREG/CR-1278 for examples of administrative control.)

8. Consult Table 4-2 to ascertain which set of conditions apply to each critical action, and for the restrictions in the number of RFs to use. No other RFs are allowed than those in Table 4-2.

9. Consult Table 4-3 to determine which of nine cases applies to each critical action. For each case, the appropriate total-failure probability $\left(F_{T}\right)$ and its upper bound (UB) are listed, exclusive of the effects of within-person dependence. For a conservative screening analysis, use the $\mathrm{F}_{\mathrm{T}} \mathrm{s}$. For an ultra-conservative screening analysis, use the UBs of the $F_{T}^{T} S$

10. Decide whether the critical human actions are performed in the context of a parallel or a series system.

a. For a series system, assess zero dependence (ZD) among the same critical human actions on different components that are in series.

b. For a parallel system assess complete dependence (CD) among the same critical human actions on different components that are in parallel. However, if a testing schedule is scheduled such that different redundant trains or components within a train are tested or restored on different shifts, assess ZD.

c. In the ASEP PRAs, other coupling of human actions were to be assessed as part of "Beta Factors," generic common-cause factors based on failure data and which were developed by systems analysts to account for unknown effects. Examples are improper maintenance of several components by the same person who used an incorrect maintenance method, or the miscalibration of several sensors resulting from faulty calibration equipment. The use of Beta Factors is not part of this screening HRA procedure. See Flemming et al (1985) for further information. (No screening analysis was used in the ASEP PRAs, including the ASEP HRAs.)

11. Enter the $\mathrm{F}_{\mathrm{T}} \mathrm{s}$ (or UBs of the $\mathrm{F}_{\mathrm{T}} \mathrm{s}$ ) in the appropriate system fault trees or system event trees, paying special attention that the dependence effects identified for human actions are preserved in the way in which the $\mathrm{F}_{\mathrm{T}} \mathrm{s}$ are used. See Chapters 5 and 10 of NUREG/CR-1278 for guidelines. 
Table 4-2 Basic and Optimum Conditions for Screening HRA of Pre-Accident Tasks, Exclusive of Within-Person Dependence Effects (p1/2)

Note 1: "Basic Conditions" refer to the absence of error recovery factors (RFs). "Optimum Conditions" refer to the presence of RFs. Each numbered Basic Condition has its same numbered complementary Optimum Condition.

\section{Basic Conditions}

Note 2: If all of the basic conditions apply (i.e., there are no RFs), the basic HEP of .03 is assessed, or, for an ultra-conservative screening analysis, its upper bound of .15 is assessed.

1. Unavailable component status is not indicated in the control room by some "compelling signal" such as an annunciator when the maintenance or calibration task or subsequent test is finished or before normal power operations can be resumed.

2. Component status is not verified by a post-maintenance (PM) or a postcalibration (PC) test; that is, it is not required or, if performed, does not verify component status.

3. There is no requirement for an $R F$ involving (1) a second person directly to verify component status after completion of a maintenance or calibration task or (2) the original performer to make a separate check of component status at a different time and place from his original task performance, or (3) the verification does not require use of a written checkoff list.

4. Shiftly or daily checks of component status (in or outside of the control room) are done without using a written checkoff list, or are not done at all. 
Table 4-2 Basic and Optimum Conditions for Screening HRA of Pre-Accident Tasks, Exclusive of Within-Person Dependence Effects (p2/2)

\section{Optimum Conditions}

Note 3: If all of the optimum conditions apply, or if optimum condition 1 only applies, a negligible HEP is assessed due to the excellence of the RFs. If an ultra-conservative screening analysis is being employed, use an HEP of .00001.

1. Unavailable component status is indicated in the control room by some "compelling signal" such as an annunciator when the maintenance or calibration task or subsequent test is finished or before normal power operation can be resumed.

2. Component status is verifiable by a PM or PC test. If done correctly, full recovery of any related error is assumed. An HEP of .01 is assessed for failure to perform the test correctly (including failure to do the test).

3. There is a requirement for an RF involving (1) a second person directly to verify component status after completion of a maintenance or calibration task, or (2) the original performer to make a separate check of component status at a different time and place from his original task performance. No credit is given for either check unless a written test is used during a check. An HEP of .1 is assessed for failure of this RF to catch an error by the original task performer. This $R F$ is presumed to be inoperative if a required PM or PC test is not performed correctly, as such failure indicates inadequate quality assurance.

4. There is a requirement for a shiftly or daily check of component status (in or outside of the control room), using a written 1ist. An HEP of .1 is assessed for the failure of such a check to detect the unavailable status. For screening purposes, this RF may be used only once per error. 
Table 4-3 Applications of Table 4-2, Exclusive of Within-Person Dependence Effects (p1/2)

Note 1: For each case below, the total failure probability, $F$, is listed with its error factor (EF) and upper bound (UB) in parentheses. The $F_{T}$ is the product of the basic HEP of .03 and the probabilities of failure of the relevant RFs. The EFs on the $F_{T}$ s were calculated using the UCBs propagation computer program described in Appendix B. For a conservative screening analysis, use the $F_{T} s$; for an ultraconservative analysis, use the UBs of the $\mathrm{F}_{\mathrm{T}} \mathrm{s}$, i.e., the $\mathrm{F}_{\mathrm{T}} \mathrm{s}$ divided by their EFs.

Note 2: In the first 4 cases, there is no "compelling signal" sas feedback. In addition, the post-maintenance (PM) or post-calibration ( $P C$ ) test is determined to be ineffective in the sense that, even if performed correctly, it will not catch the original error.

Case I - PM or PC Test not effective; no other RFs used:

a. All Basic Conditions apply.

b. $\quad \mathrm{BHEP}=.03=\mathrm{F}_{\mathrm{T}} . \quad(\mathrm{EF}=5, \mathrm{UB}=.15)$.

Case II - No compelling signal feedback; PM or PC Test not effective; both other RFs used:

a. Basic Conditions 1, 2 apply.

b. Optimum Conditions 3,4 apply.

c. $\mathrm{F}_{\mathrm{T}}=.03 \times .1 \times .1=.0003 .(\mathrm{EF}-16, \mathrm{UB}-.005)$.

Case III - No compelling signal feedback; PM or PC Test not effective; second person or other immediate RF used:

a. Basic Conditions 1, 2, 4 apply.

b. Optimum Condition 3 applies.

c. $\mathrm{F}_{\mathrm{T}}=.03 \times .1=.003 .(\mathrm{EF}-10, \mathrm{UB}-.03)$.

Case IV - No compelling signal feedback; PM or PC Test not effective; periodic check is made:

a. Basic Conditions 1, 2, 3 apply.

b. Optimum Condition 4 applies.

c. $\mathrm{F}_{\mathrm{T}}=.03 \times .1=.003 .(\mathrm{EF}-10, \mathrm{UB}=.03)$. 
Table 4-3 Applications of Table 4-2,

Exclusive of Within-Person Dependence Effects (p2/2)

Note 3: In the last 5 cases, the PM or PC Test is determined to be effective, i.e., if performed correctly it will detect the original error.

Case V - Original error is annunciated; all other optimum conditions are immaterial:

a. At least Optimum Condition \#1 applies.

b. $\mathrm{F}_{\mathrm{T}}=$ negligible. (Assess UB of .00001).

Case VI - PM or PC Test is effective if performed correctly; no other RFs used:

a. Basic Conditions 1, 3, 4 apply.

b. Optimum Condition 2 applies.

c. Probability of not performing, or not performing correctly, the required $\mathrm{PM}$ or $\mathrm{PC}$ Test $=.01$

d. $\mathrm{F}_{\mathrm{T}}=.03 \times .01=.0003 . \quad(\mathrm{EF} \sim 10, \mathrm{UB}=.003)$.

Case VII - No compelling signal feedback; PM or PC Test is effective if performed correctly; both other RFs are used:

a. Basic Condition 1 applies .

b. Optimum Conditions 2, 3, 4 apply.

c. $\mathrm{F}_{\mathrm{T}}=.03 \times .01 \times 1.0 \times .1=.00003 .(\mathrm{EF} \sim 16, \mathrm{UB} \sim .0005)$.

(Note: The 1.0 means no recovery credit is given for Optimum Condition 3 if the PM or PC Test is not done or done correctly per Optimum Condition 2.)

Case VIII - No compelling signal feedback; PM or PC Test is effective if performed correctly; second person or other immediate RF is used:

a. Basic Conditions 1, 4 apply.

b. Optimum Conditions 2, 3 apply.

c. $\mathrm{F}_{\mathrm{T}}=.03 \times .01 \times 1.0=.0003 .(\mathrm{EF} \sim 10, \mathrm{UB}=.003)$.

Case IX - No compelling signal feedback; PM or PC Test is effective if performed correctly; periodic check is made:

a. Basic Conditions 1, 3 apply.

b. Optimum Conditions 2, 4 apply.

c. $\mathrm{F}_{\mathrm{T}}=.03 \times .01 \times .1=.00003 .(\mathrm{EF}-16, \mathrm{UB}-.0005)$. 
CHAPTER 5. ASEP NOMINAL HRA FOR PRE-ACCIDENT TASKS

\section{General Information}

Much of the procedure for the pre-accident nominal human reliability analysis (HRA) for ASEP is similar to the screening HRA in Chapter 4. For example, steps $1-8$ and 11 of Table 5-1, the Procedure for Nominal HRA of Pre-Accident Tasks, are almost identical to steps in Table 4-1, the equivalent table for the screening procedure. The major difference between the screening and nominal HRA procedures is the latter's greater credit for recovery factors (RFs) and a more detailed consideration of dependence effects. However, in the procedures for the nominal HRA, the human error probabilities (HEPs) assigned to failure of RFs represent a more conservative assessment than that which would be obtained by use of the THERP/Handbook approach given in NUREG/CR-1278. The treatment of dependence in the ASEP nominal HRA procedure for pre-accident tasks represents a simplification of the positive dependence model in Chapter 10 of NUREG/CR-1278. As usual, such simplification results in some loss of accuracy, and is compensated for by making the dependence assessments more conservative than would be the case when using the dependence mode1 in NUREG/CR-1278.

Most of the HEPs in the tables are total failure probabilities, $F_{T} s$, which represent the basic HEP of .03 for some original error multiplied by failures of various recovery factors. The tables also include the error factor (EF) for each $F_{T}$ to permit the calculations of upper uncertainty bounds (UBs) and lower uncertainty bounds (LBs) of the $\mathrm{F}_{\mathrm{T}} \mathrm{s}$. To obtain the UB, multiply the $\mathrm{F}_{\mathrm{T}}$ by the EF; to obtain the LB, divide the $F_{T}$ by the EF. (The EFs were calculated using the uncertainty bounds propagation computer program described in Appendix B.) The LBs can be used to conduct a sensitivity analysis. For example, assume that the HRA results for several tasks using the nominal values have a material impact on the systems analysis. In a sensitivity analysis, the LBs of the appropriate $F_{T}$ terms can be used instead of the nominal $F_{\mathrm{T}}$ values to see if the lower values would have no material effect in the systems analysis. If it were found that the lower values for some tasks had no effect, this could provide a rationale for a more detailed HRA to be performed per NUREG/CR-1278 to see how low the estimated $F_{T^{s}}$ would be (1) when full credit is given to all of the recovery factors and (2) when better estimates are made of the basic HEPs than the gross value of .03 selected for the original HRA using the ASEP HRA Procedure.

Another purpose of the uncertainty bounds (UCBs) is to provide a basis for a Monte Carlo procedure. The upper and lower bounds on the $\mathrm{F}_{\mathrm{T}}$ estimates are intended to represent $95 \%$ and $5 \%$ error probabilities on a presumed lognormal scale of error probabilities, with the nominal $\mathrm{F}_{\mathrm{T}}$ value representing the 
median on this distribution. The rationale for these assumptions is presented in Chapter 7 of NUREG/CR-1278. If these assumptions are acceptable for the PRA in question, the values in the tables can be used for the above purpose.

\section{Discussion of ASEP Dependence Mode1}

There are two types of deperidence used in the ASEP HRA Procedure: betweenperson dependence and within-person dependence. Between-person dependence is handled in the treatment of RFs in the basic procedure in Table 5-1. Withinperson dependence is handled with a new dependence model described below. Within-person dependence refers to the dependence among human actions by one person who is performing operations on more than one component. The components can constitute a series system or a parallel system, for example, two pumps which are both needed to accomplish a necessary function (a series system) or two pumps, either one of which will accomplish a necessary function (a parallel system). In the case of the series system, one human error, e.g., forgetting to start either pump, will prevent the necessary function from being activated. In the case of the parallel system, two human errors would be required to defeat the function, e.g., forgetting to start both pumps.

The ASEP Nominal HRA Procedure includes a new model for assessing withinperson dependence for pre-accident tasks. The dependence model in NUREG/CR1278 has five levels of positive dependence: zero dependence (ZD), low dependence (LD), moderate dependence (MD), high dependence (HD), and complete dependence (CD). The ASEP model is also a positive dependence model, but it uses only three of these levels, $Z D, H D$, and $C D$, and makes additional simplifications. No provision is made in this model for assessing negative dependence, as in nearly all HRA applications, such an assessment leads to unbelievably low estimates of joint HEPs (i.e., the product of the HEP for the first task and the conditional HEPs derived by use of negative dependence). (See Chapter 10 in NUREG/CR-1278 for the rationale behind this statement.)

The ASEP dependence model is presented in two formats. Figure 5-1 presents the model in the form of a binary decision tree. Table 5-4, later in this chapter, uses the results of this tree to provide tabled guidelines for assessing within-person dependence levels.

Following is a discussion of Figure 5-1, starting with the top of the table and working downwards. The first decision node, Series System?, asks whether the same critical human actions on different components are for components that constitute a series system. If the answer is yes, $Z D$ is assessed for both errors of omission (EOMs) and errors of commission (ECOMs). This means, of course, that ZD is assessed for the BHEP of .03. If the answer is no, this is equivalent to stating that the different components constitute a parallel system, and leads to the next decision node, ECOM? The Yes path under this decision node means that for parallel systems, ZD is assessed for the ECOM part of the BHEP of .03, i.e., for the .01 that is the assessed contribution of an ECOM, as discussed in Chapter 3. The No path is equivalent to assessing dependence for the EOM part of the BHEP of .03, i.e., the .02 that is the 

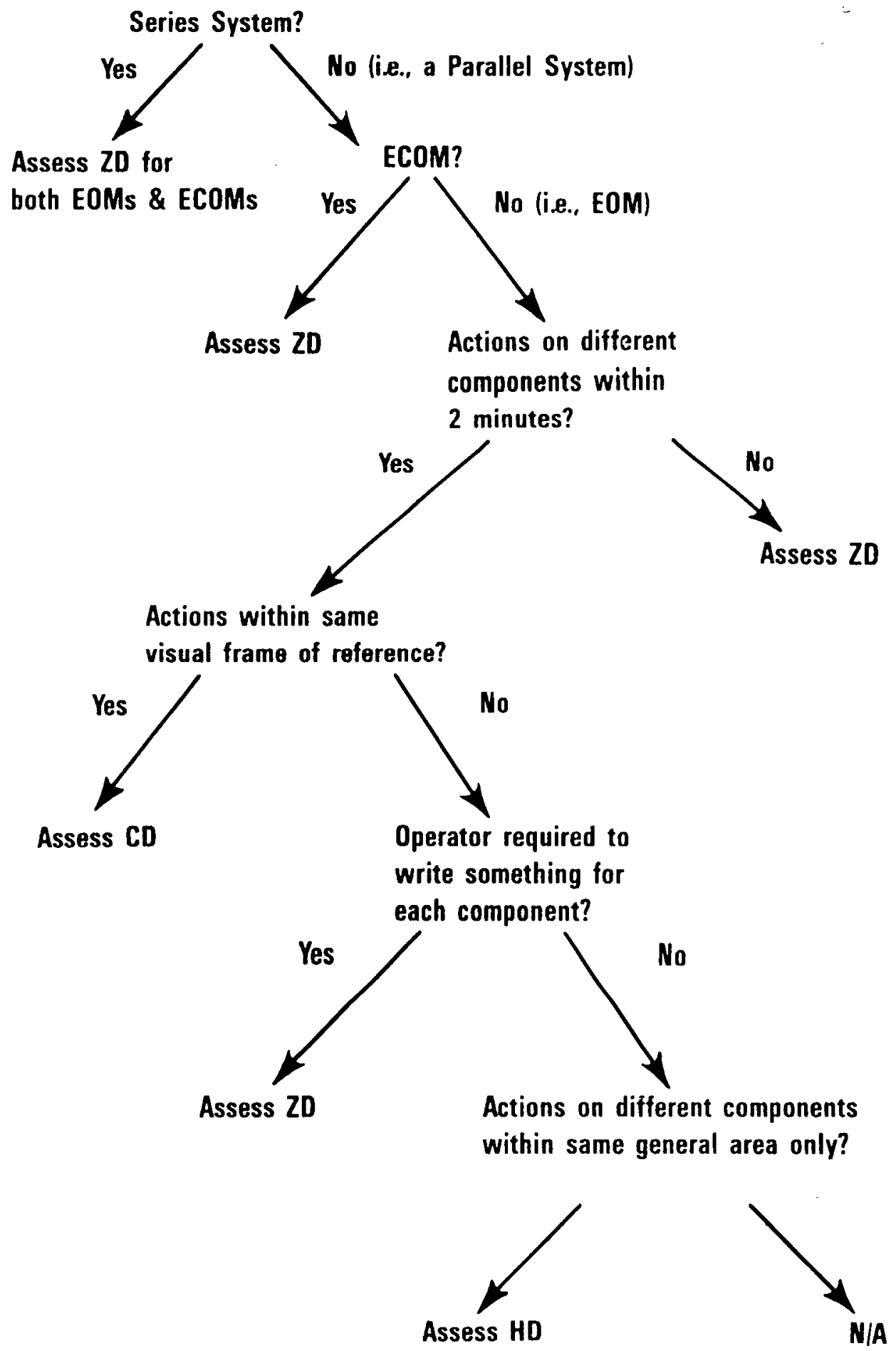

Figure 5-1 ASEP Model for Assessing Within-Person Positive Dependence Levels for Nominal HRA of Pre-Accident Tasks 
assessed contribution of an EOM. The remainder of the tree deals with assessing $\mathrm{ZD}, \mathrm{HD}$, or $\mathrm{CD}$ for the EOM portion of the BHEP. Step 10 in the basic procedure in Table 5-1 refers to Tables 5-4 and 5-5 in which provision is made to handle the EOM and ECOM aspects of a task in a parallel system.

The next decision node, Actions on different components within 2 minutes?, deals with closeness in time. If the actions are not close in time (i.e., within two minutes), $Z D$ is assessed the EOM portion. If the actions take place in a time span of two minutes or less, the next decision node, Actions within same visual frame of reference?, refers to closeness in spatial separation. Components are defined as being in the same frame of reference if both are in view without head movement, as the operator is performing an action on one of them. If the answer is yes, $C D$ is assessed for the EOM portion of the BHEP. A no answer leads to the next decision node, Operator required to write down some information pertaining to each component? Here, if the answer is yes, $C D$ is assessed. A no answer leads to the final decision node, Actions on different components within same general area only? Components in the "same general area" are considered to be no farther apart than a few steps. If the answer is yes, $H D$ is assessed. The No path is designated as not applicable (N/A); it is assumed to define an empty set because, in general, actions that take place within two minutes of each other should be at least in the same general area.

Following is additional, partly redundant discussion of the ASEP dependence model intended to assist the user in how it should be applied, with reference to the tables making up the procedure for the nominal HRA for pre-accident tasks.

First, $Z D$ is always assessed among the critical human actions on different components that are in series. Therefore, the systems analyst can use the rules and $\mathrm{F}_{\mathrm{T}} \mathrm{s}$ in Table 5-3 with appropriate modification for the number of components in the system, as provided for in Table 5-5.

For parallel systems, it is necessary for the systems analyst to first identify those pre-accident tasks of interest for which ZD can be assessed for the EOM portions of the tasks. For those tasks for which ZD is assessed, the above rules and $\mathrm{F}_{\mathrm{T}} \mathrm{s}$ in Table 5-3 are relevant, with appropriate modification for the number of components in the system, as provided for in Table 5-5. ZD is assessed among the same critical actions on parallel components if the actions do not occur closely in time, i.e., the between-action interval for each pair of related actions is more than 2 minutes. The two-minute rule was adopted as a conservative modification of the one-minute guideline discussed under the heading "Functional Relationships Among Tasks" beginning on page 1019 of NUREG/CR-1278. ZD is also assessed if there is good physical separation of the components in question (i.e., the components are not in the same visual frame of reference) and the operator is supposed to write down some information pertaining to each component in question, not just making a check mark or writing down ones initials. Any two components in a related group are considered to be in the same visual frame of reference if the operator can see one of them without moving his head as he is performing some action on the other. This assessment of ZD for same type critical actions related to components with good physical separation may be made even if the actions required 
for each well-separated component occur closely in time. (In the ASEP probabilistic risk assessment (PRA) of the Grand Gulf boiling water reactor (BWR) nuclear power plant (NPP) in Volume 6 of NUREG/CR-4550, the "same frame of reference" was defined as components being within 4 feet of each others. While this definition is not as conservative as the one above, the difference is probably not important, and the "within 4 feet" rule is easier to apply.)

For parallel system applications, the assessment of ZD is only slightly less conservative than a non-zero level of positive dependence, i.e., $F_{T} s$ will be slightly smaller if $\mathrm{ZD}$ is assessed. For this reason, then, there should be a sound rationale for the assessment of the zero level of dependence when assessing parallel systems.

Next, for parallel systems, the systems analyst must identify those tasks for which non-zero levels of positive dependence are to be assessed. For conservatism, only two non-zero levels of positive dependence are used of the four levels identified in Chapter 10 of NUREG/CR-1278. The two non-zero levels used are complete dependence (CD) and high dependence (HD). Assessments of low dependence (LD) and moderate dependence (MD) are not made. CD is assessed if the components in question are within the same visual frame of reference, whether or not the operator is supposed to write down some information about each component, and the between-action interval for each set of related actions is 2 minutes or less. HD is assessed if the between-action interval for each set of related actions is 2 minutes or less, the components in question are in the same general area, but not within the same visual frame of reference, and there is no requirement for the operator to write information about each component. For example, HD would be assessed if the physical separation and time separation are as stated, and the operator either writes down nothing or he makes check marks or uses his initials to check off that steps in the procedure have been accomplished. (In the ASEP PRA of the Grand Gulf BWR NPP, this intermediate dependence level was not assessed. If ZD could not be assessed, CD was assessed, resulting in additional conservatism for parallel system applications.) For simplicity, it is assumed that the level of dependence in any set of related actions remains constant. For example, in a threecomponent parallel system, if $C D$ is assessed between the first and second components, $\mathrm{CD}$ should also be assessed between the second and third components. Table 5-4 summarizes these guidelines for assessing dependence.

\section{The Procedure}

Table 5-1 presents the steps to follow in applying the ASEP Nominal HRA Procedure for pre-accident tasks, and Tables 5-2, 5-3, 5-4, and 5-5 present the tables of estimated HEPs and other rules for this procedure. For background material used to develop these tables, including the rationale for the basic HEP of .03, see Swain (1985a) or the section on "Screening Rules for the Human Reliability Analysis in the Risk Methods and Evaluation Program" in Volume 5 of NUREG/CR-4832 (Payne et al, 1987). 
Table $5=1$, Procedure for Nominal HRA of Pre:Accident Tásks (p1/4)

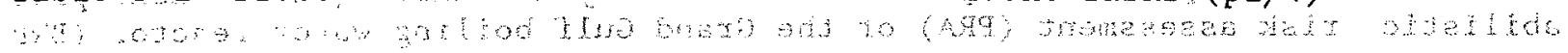

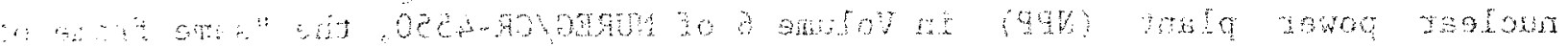

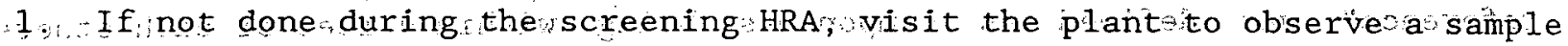
a of prefaccident tasks (especially ycalibration tasks ipost clalibration and post-maintenance tests, and restoration tasks) and obtain elevant written procedures and other documentation that spells out operating se-

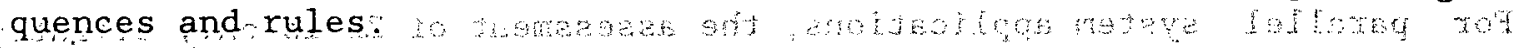

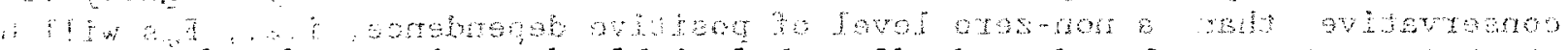
a The observations shouldinclude talk-throughs of several preaccident procedures as they are being performed emphasizing restoration tasks, and, if possible, some actual calibration tasksqand postcalibration and post-maintenance tests. Failure to make these observations, including interviews with operating personnel who actually perform the tasks will mean that the estimated HEPs will probably

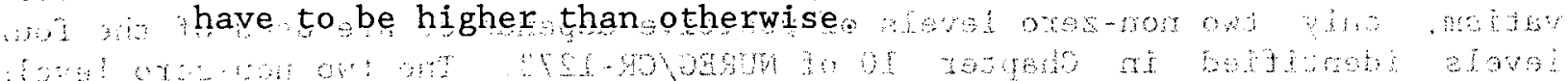
a b Carefully evaluate the quality of the administrative contro1, eg. tsa how well wescribedopre-accident tasks, especially sore recovery factors $(R F s)$, will be performed a (See Chapteri 16 in

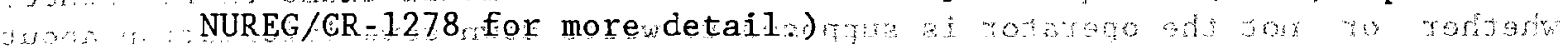

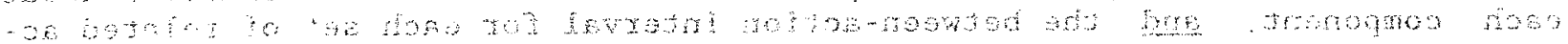

is Additional plant visits maybe necessary once it has been determined which preaccident tasks have a material effect in theoverall sys -

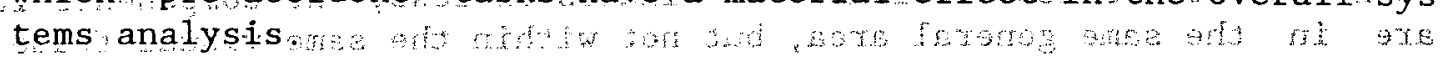

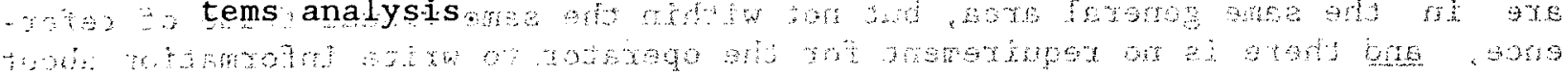

2. Based on the information obtained in step 1, mdetermine if the basichEP of 03 should be adjusted upwards for tunusually poor humanofactors, a especially written procedures a no downward adjustmént should bé mäde without a more thorough HRA of therkind specified in NUREG\%CR $127.8 \% 00 \times$

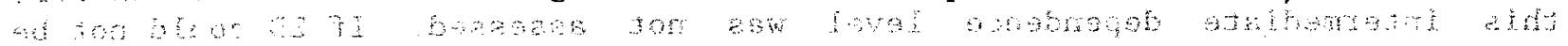

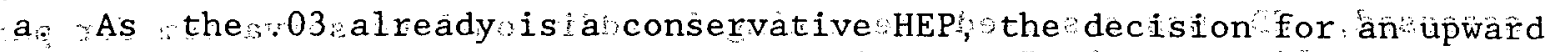
adjustment should be fully documented The human factors in a pant - ast would have to be unusually pooretorequirean upward adjustment. ort

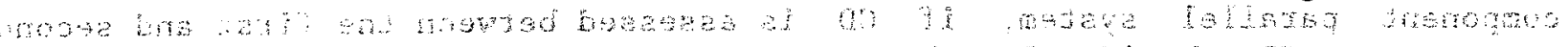

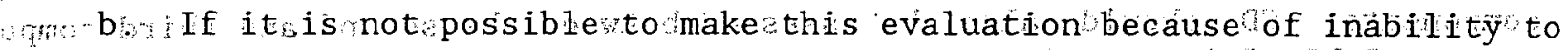
cobserve pre-accident taskś (or todureceivetalk through of these tasks) or because of inability to evaluate adequately the administrative control procedures, employ a .05 basic HEP in place of the .03 HEP. All the other numbers in the tables which are based on the .03 HEP will have to be changed, using the arithmetic implied by the tables.

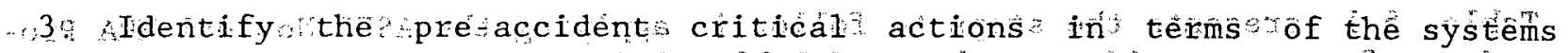

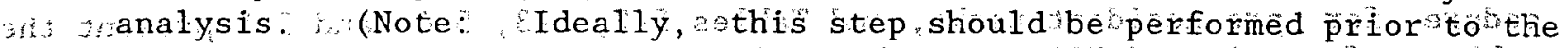

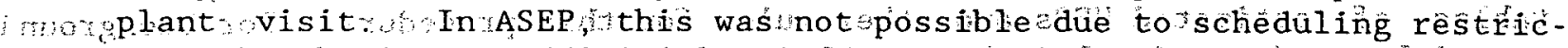

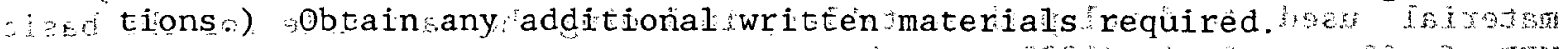

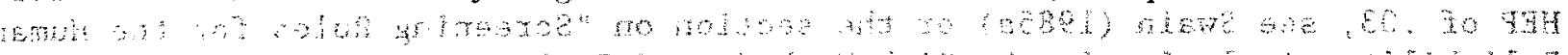

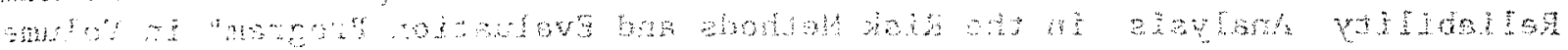

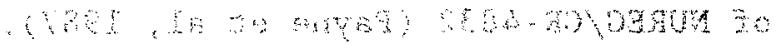


Table 5-1 Procedure for Nominal HRA of Pre-Accident Tasks ( $\mathrm{p} 2 / 4)$

4. Identify the recovery factors (RFs) listed below. Do not consider any other RFs.

a. Determine for which critical pre-accident actions, errors are fully recoverable by "compelling signals," usually one or more annunciators

" when a maintenance or calibration task is completed or before normal power operation can be resumed.

b. Determine for which critical pre-accident actions, errors can be recovered by a post-maintenance (PM) or post-calibration ( $P C$ ) test if the test is performed correctly. (Just because a test is scheduled does not guarantee that it will be performed, and performed correctly.)

c. Determine for which critical pre-accident actions, (1) a second person is required to directly verify component status after completion of the actions by the original performer, or (2) the original performer is required to make a separate check of component status at a different time and place from his original performance. An example of the latter would be for an operator to restore a locally-operated valve to its normal operating condition following maintenance, and then to return to the control room and note that an appropriate indicator light shows the desired position of the valve. No recovery credit is given for either check unless a written checkoff list is used during the check.

d. Determine for which critical pre-accident actions there is a requirement for a shiftly or daily check of component status in or outside of the control room, using a written checkoff list. No recovery credit is given for either check unless a written checkoff list is used during the check.

e. For supervisor sign-off without his actually visually checking each component in question, give $R F$ credit for the entire pre-accident job having been initiated, but not for the accuracy or completeness of any task or step in the job. That is, this RF does not constitute a check on errors of omission (EOMs) or errors of commission (ECOMs) for individual actions in the job.

5. Assign a basic HEP (BHEP) of .02 for each EOM and .01 for each ECOM. Assume that an ECOM is always possible if an EOM is not made. Therefore, for each critical action, assign a total BHEP of .03. 
Table 5-1 Procedure for Nominal HRA of Pre-Accident Tasks (p3/4)

6. Assign a .1 HEP for failure of each relevant RF, except for a .01 failure to perform a required PM or PC test or to perform it correctly. The RF already includes between-person dependence, and is assumed to apply to the EOM and ECOM as a unit, i.e., to the BHEP of .03 for a complete critical action. Therefore, it is appropriate to multiply the .03 by the product of the RF HEPs, and treat the final product as a unit. It is judged that the assessed probability of failure of each RF is high enough to also include estimated failures of administrative contro1, i.e., the failure to perform the prescribed RF. (See Chapter 16 in NUREG/CR-1278 for examples of administrative control.)

7. Consult Table 5-2 to ascertain which set of conditions apply to each critical action, and for the restrictions in the number of RFs to use. With the following two exceptions, no other RFs are allowed than those in Table 5-2.

a. The .1 HEP for failure of a supervisor sign-off to detect that an entire set of pre-accident tasks was not initiated should be considered. separately when constructing the HRA event trees. (Note: For conservatism, this RF was not employed in the ASEP PRAs.)

b. If any task has a material effect in the overall systems analysis, and this task had an RF based on a shiftly or daily written check, a more detailed HRA can be performed, giving the appropriate credit for the shiftly or daily check, rather than just counting it once per Table 5-2. If this approach is followed, carefully evaluate the possibility of any dependence among the checks, i.e., justify any assumption of zero dependence.

8. Consult Table 5-3 to determine which of nine cases applies to each critical action. For each case, the appropriate total-failure probability $\left(F_{T}\right)$ and its error factor (EF) are listed, exclusive of the effects of within-person dependence.

a. For the nominal HRA, use the $\mathrm{F}_{\mathrm{T}} \mathrm{s}$ in Tables 5-3 and 5-5. For a Monte Carlo procedure, the distribution for each $\mathrm{F}_{\mathrm{T}}$ can be used, assuming the listed $F_{T}$ is the median on a lognormal curve and the $U B$ and $L B$ represent, respectively, the 958 and 58 HEPs.

b. In the ASEP PRAs, a few other cases involving dependence of human actions was assessed as part of "Beta Factors," generic common-cause factors based on failure data and which were developed by systems analysts to account for dependence effects. Examples are improper maintenance of several components by the same person who used an incorrect maintenance method, or the miscalibration of several sensors resulting from faulty calibration equipment. The use of Beta Factors is not part of this nominal HRA procedure. See Flemming et al (1985) for further information. 
Table 5-1 Procedure for Nominal HRA of Pre-Accident Tasks (p4/4)

9. Decide whether the critical human actions are performed in the context of a parallel or a series system.

a. For a series system, assess zero dependence (ZD) among the same critical human actions on different components that are in series.

b. To obtain required information for assessing within-person dependence in a parallel system, determine which components of interest are within the same visual frame of reference (i.e., those which are in view without head movement as an operator is performing an action on one of them), within the same general area, or not within the same general area, and/or which operator actions are close in time (i.e., less than 2 minutes). If information on physical separation cannot be obtained, assume the components are in the same visual frame of reference. If information on time cannot be obtained, assume the actions occur closely in time.

c. Consult Table 5-4 to assess the levels of dependence between the critical human actions performed by an operator which are related to the components in question. For simplicity, assume that the level of dependence in any set of human actions on related components remains constant. Note that $\mathrm{ZD}$ is assumed for human actions related to series systems. As explained in Chapter 10 of NUREG/CR-1278, this assumption provides a small (usually immaterial) increase in conservatism for the relatively small basic HEP of .03. Note that in parallel systems, $\mathrm{ZD}$ is assumed for the ECOM portion (i.e., .01) of the basic HEP of .03, and ZD, high dependence (HD), or complete dependence (CD) is assessed for the EOM portion (i.e., .02) of the basic HEP of .03. Low dependence (LD) and moderate dependence (MD) (from the dependence model in NUREG/CR-1278) are not used. For the usual applications, this omission results in increased conservatism.

10. Consult Table 5-5 to determine the $\mathrm{F}_{\mathrm{T}} \mathrm{s}$ (or UBs or LBs of the $\mathrm{F}_{\mathrm{T}} \mathrm{s}$ ) for any of the nine cases (in Table 5-3) relevant to the analysis, as modified for multiple-component systems and for the effects of dependence (from Table 5-4). Note that for some dependence assessments, the contribution of ECOMs are ignored, and only that of EOMs are used. This dependence procedure is more conservative than that found in Chapter 10 of NUREG/CR1278 .

11. Enter the $F_{T} s$ (or UBs or estimated distribution parameters of the $F_{T} s$ ) in the appropriate system fault trees or system event trees, paying special attention that the dependence effects identified for human actions are preserved in the way in which the $\mathrm{F}_{\mathrm{T}} \mathrm{s}$ are used. See Chapters 5 and 10 of NUREG/CR-1278 for guidelines. 
Table 5-2 Basic and Optimum Conditions for Nominal HRA of Pre-Accident Tasks, Exclusive of Within-Person Dependence Effects (p1/2)

Note 1: "Basic Conditions" refer to the absence of error recovery factors (RFs). "Optimum Conditions" refer to the presence of RFs. Each numbered Basic Condition has its same numbered complementary Optimum Condition.

\section{Basic Conditions}

Note 2: If all of the basic conditions apply (i.e., there are no RFs), the basic HEP of .03 with an EF of 5 is assessed.

1. Unavailable component status is not indicated in the control room by some "compelling signal" such as an annunciator when the maintenance or calibration task or subsequent test is finished or before normal power operations can be resumed.

2. Component status is not verified by a post-maintenance (PM) or a postcalibration (PC) test; that is, it is not required or, if performed, does not verify component status.

3. There is no requirement for an RF involving (1) a second person directly to verify component status after completion of a maintenance or calibration task or (2) the original performer to make a separate check of component status at a different time and place from his original task performance, or (3) the verification does not require use of a written checkoff list.

4. Shiftly or daily checks of component status (in or outside of the control room) are done without a written checkoff list, or are not done at all. 
Table 5-2 Basic and Optimum Conditions for Nominal HRA of Pre-Accident Tasks, Exclusive of Within-Person Dependence Effects (p2/2)

\section{Optimum Conditions}

Note 3: If all of the optimum conditions apply, or if optimum condition 1 only applies, a negligible HEP is assessed due to the excellence of the RFs. Use an upper bound (UB) of .00001.

1. Unavailable component status is indicated in the control room by some "compelling signal" such as an annunciator when the maintenance or calibration task or subsequent test is finished or before normal power operation can be resumed.

2. Component status is verifiable by a PM or PC test. If done correctly, full recovery of any related error is assumed. An HEP of .01 is assessed for failure to perform the test correctly (including failure to do the test).

3. There is a requirement for an RF involving (1) a second person directly to verify component status after completion of a maintenance or calibration task, or (2) the original performer to make a separate check of component status at a different time and place from his original task performance. No credit is given for either check unless a written checkoff list is used during a check. An HEP of . 1 is assessed for failure of this RF to catch an error by the original task performer. This RF is presumed to be inoperative if a required $\mathrm{PM}$ or $\mathrm{PC}$ test is not performed correctly, as such failure indicates inadequate quality assurance.

4. There is a requirement for a shiftly or daily check of component status (in or outside of the control room), using a written checkoff list. An HEP of .1 is assessed for the failure of such a check to detect the unavailable status. For the initial nominal HRA, this RF may be used only once per error. If this conservatism results in a task's having a material effect in the system analysis, perform a more detailed analysis, giving credit for the daily or shiftly schedule, per NUREG/CR-1278. 
Table 5-3 Applications of Table 5-2, Exclusive of Within-Person Dependence Effects (p1/2)

Note 1: For each case below, the total failure probability, $F_{T}$, is listed with its error factor (EF) in parentheses. The $F_{T}$ is the product of the basic HEP of .03 and the probabilities of failure of the relevant RFs. The EFs on the $F_{T} s$ were calculated using the UCBs propagation computer program described in Appendix B. To calculate the upper uncertainty bound (UB) and lower uncertainty bound (LB), multiply and divide each $\mathrm{F}_{\mathrm{T}}$ by its $\mathrm{EF}$.

Note 2: In the first 4 cases, there is no "compelling signal" as feedback. In addition, the post-maintenance ( $P M$ ) or post-calibration ( $P C$ ) test is not effective in the sense that, even if performed correctly, it will not catch the original error.

Case I - PM or PC Test not effective; no other RFs used:

a. All Basic Conditions apply.

b. $\mathrm{BHEP}=.03=\mathrm{F}_{\mathrm{T}} . \quad(\mathrm{EF}=5)$.

Case II - No compelling signal feedback; PM or PC Test not effective; both other RFs used:

a. Basic Conditions 1, 2 apply.

b. Optimum Conditions 3,4 apply.

c. $\mathrm{F}_{\mathrm{T}}=.03 \times .1 \times .1=.0003 .(\mathrm{EF}-16)$.

Case III - No compelling signal feedback; PM or PC Test not effective; second person or other immediate RF used:
a. Basic Conditions 1, 2, 4 apply.
b. Optimum Condition 3 applies.
c. $\mathrm{F}_{\mathrm{T}}=.03 \times .1=.003 .(\mathrm{EF}-10)$.

Case IV - No compelling signal feedback; PM or PC Test not effective; periodic check is made:

a. Basic Conditions 1, 2, 3 apply.

b. Optimum Condition 4 applies.

c. $\mathrm{F}_{\mathrm{T}}=.03 \times .1=.003 .(\mathrm{EF}-10)$. 
Table 5-3 Applications of Table 5-2, Exclusive of Within-Person Dependence Effects (p2/2)

Note 3: In the last 5 cases, the PM or PC Test is effective, i.e., if performed correctly it will detect the original error.

Case V - Original error is annunciated; other optimum conditions are immaterial:

a. At least Optimum Condition \#1 applies.

b. $\mathrm{F}_{\mathrm{T}}=$ negligible. (Assess UB of .00001).

Case VI - PM or PC Test is effective if performed correctly; no other RFs used:

a. Basic Conditions 1, 3, 4 apply.

b. Optimum Condition 2 applies.

c. Probability of not performing or not performing correctly required PM or PC Test $=.01$

d. $\quad \mathrm{F}_{\mathrm{T}}=.03 \times .01=.0003 .(\mathrm{EF}-10)$.

Case VII - No compelling signal feedback; PM or PC Test is effective if performed correctly; both other RFs are used:

a. Basic Condition 1 applies .

b. Optimum Conditions 2, 3, 4 apply.

c. $\mathrm{F}_{\mathrm{T}}=.03 \times .01 \times 1.0 \times: 1=.00003 .(\mathrm{EF}-16)$.

(Hote: The 1.0 means no recovery credit is given for Optimum Condition 3 if the PM or PC Test is not done or done correctly per Optimum Condition 2.)

Case VIII - No compelling signal feedback; PM or PC Test is effective if performed correctly; second person or other immediate RF is used:

a. Basic Conditions 1, 4 apply.

b. Optimum Conditions 2, 3 apply.

c. $\mathrm{F}_{\mathrm{T}}=.03 \times .01 \times 1.0=.0003 .(\mathrm{EF}-10)$.

Case IX - No compelling signal feedback; PM or PC Test is effective if performed correctly; periodic check is made:

a. Basic Conditions 1, 3 apply.

b. Optimum Conditions 2, 4 apply.

c. $\mathrm{F}_{\mathrm{T}}=.03 \times .01 \times .1=.00003 .(\mathrm{EF}-16)$. 
Table 5-4 Guidelines for Assessing Within-Person Dependence Levels for Nominal HRA of Pre-Accident Tasks

SERIES SYSTEMS:

Assume ZD for both EOMs and ECOMs

PARALLEL SYSTEMS :

Errors of Omission (EOMs)

For the Group of Components in Question:

\begin{tabular}{|c|c|c|c|c|c|c|c|c|}
\hline \multirow{5}{*}{$\begin{array}{c}\text { Level } \\
\text { of } \\
\text { Dependence }\end{array}$} & & & \multicolumn{4}{|c|}{ Located in Same: } & \multicolumn{2}{|c|}{ Operator } \\
\hline & \multicolumn{2}{|c|}{ Actions } & \multicolumn{2}{|c|}{ Visual } & \multicolumn{2}{|c|}{ General } & \multicolumn{2}{|c|}{ Write Something } \\
\hline & \multicolumn{2}{|c|}{ Close in } & \multicolumn{2}{|c|}{ Frame of } & \multicolumn{2}{|c|}{ Area } & \multirow{2}{*}{\multicolumn{2}{|c|}{$\begin{array}{l}\text { for Each } \\
\text { Component }\end{array}$}} \\
\hline & \multicolumn{2}{|c|}{ Timex } & \multicolumn{2}{|c|}{ Reference** } & \multicolumn{2}{|c|}{ On1y } & & \\
\hline & YES & NO & YES & NO & YES & No & YES & No \\
\hline \multirow{2}{*}{ ZD } & & $\mathrm{x}$ & \multicolumn{2}{|c|}{ either } & \multicolumn{2}{|c|}{ either } & \multicolumn{2}{|c|}{ either } \\
\hline & \multicolumn{2}{|l|}{$\mathrm{x}$} & & $\mathrm{x}$ & \multicolumn{2}{|c|}{ either } & \multicolumn{2}{|l|}{$\mathrm{x}$} \\
\hline $\mathrm{HD}--$ & \multicolumn{2}{|l|}{$\mathrm{x}$} & & $\mathrm{x}$ & \multicolumn{2}{|l|}{$\mathrm{x}$} & \multicolumn{2}{|r|}{$\mathrm{x}$} \\
\hline$C D--$ & \multicolumn{2}{|l|}{$\mathrm{x}$} & $\mathrm{x}$ & & \multicolumn{2}{|c|}{ irrelevan } & \multicolumn{2}{|c|}{ either } \\
\hline
\end{tabular}

Errors of Commission (ECOMs)

Assume ZD Regardless of Conditions

\footnotetext{
* Actions are considered to be close in time if the actions required for each component in the group are separated by 2 minutes or less.

Two components are in the same frame of reference if both are in view without head movement, as the operator is performing an action on one of them. In one ASEP PRA, the same frame of reference was defined as components being within 4 feet of each other. This definition is not as conservative as that in the first sentence in this footnote, but the difference is probably not important, and the "within 4 feet" rule is easier to apply.
} 
Table 5-5 $\mathrm{F}_{\mathrm{T}} \mathrm{s}$ for Table 5-3 BHEPs, Modified for Multiple-Component Systems, Assuming Dependence Levels Determined by Using Guidelines in

Table 5-4, and Including RFs ( $1 / 3$ )

Note 1: Scientific notation is used in this table to save space.

Note 2: The upper bounds (UBs) and lower bounds (LBs) are calculated by multiplying and dividing the $F_{T} s$ by the error factors (EFs) which are listed in parentheses. The EFS were calculated using the UCBs propagation method described in Appendix B.

Note 3: If $\mathrm{ZD}$ can be assessed for the EOMs in a parallel system, $\mathrm{F}_{\mathrm{T}}=\left(.03 \times \mathrm{F}_{\mathrm{rf}}\right)^{\mathrm{n}}$,

where .03 is the basic HEP combining the contribution of EOM (HEP = .02 ) and ECOM (HEP $=.01$ ) for one component, $\mathrm{F}_{\mathrm{rf}}$ is the probability of failure of the relevant RFs, and $n$ is the number of components in the system. With the assumption of ZD for ECOMs (per Table 5-4), the ECOM HEP of .01 contributes materially to $F_{T}$ in a parallel system. The UBs and LBs for the median HEPs related to ZD in a parallel system are listed in parentheses following each HEP:

$.01(.03-.003) ; .02(.1-.004) ; .03(.15-.006) ; .1(.5-.02)$.

Note 4: If other than $\mathrm{ZD}$ is assessed for the EOMs in a parallel system, ignore the ECOMs, as they do not contribute materially to $\mathrm{F}_{\mathrm{T}}$, and calculate the $\mathrm{F}_{\mathrm{T}} \mathrm{s}$ for assessments of $\mathrm{CD}$ and $\mathrm{HD}$ as follows,

$F_{T} \mid C D=.02 \times F_{r f} \times 1.0^{n-1}=.02 \times F_{r f}$

$\mathrm{F}_{\mathrm{T}} / \mathrm{HD}=.02 \times \mathrm{F}_{\mathrm{rf}} \times \cdot 5^{\mathrm{n}-1}$

where $.02, F_{\text {f }}$, and $\mathrm{n}$ are defined per Note 3 , and 1.0 and .5 are the conditional HEPs for the second or more human actions following the basic EOM. The UBs and LBs for the median HEPs related to a CD and $\mathrm{HD}$ in a parallel system are listed in parentheses following each HEP:

$.01(.03-.003) ; .02(.1-.004) ; .1(.5-.02) ; 1.0(1.0-1.0)$. 
Table 5-5 $\mathrm{F}_{\mathrm{T}} \mathrm{s}$ for Table 5-3 BHEPs, Modified for Multiple-Component Systems, Assuming Dependence Levels Determined by Using Guidelines in Table 5-4, and Including RFs (p2/3)

Note 5: If $Z D$ is assessed for the human actions in a series system, use the basic HEP of .03 (EOM + ECOM), and employ an approximate failure equation as follows:

$\mathrm{F}_{\mathrm{T}}-\mathrm{n}\left(.03 \times \mathrm{F}_{\mathrm{rf}}\right)$

where $.03, n$, and $F_{\text {f }}$ are defined per Note 3 . The UBs and LBs for the three median HEPS in the table related to a series system are listed in parentheses following each HEP:

$.01(.03-.003) ; .03(.15-.006) ; .1(.5-.02)$.

Note 6: In the first 4 cases below, the PM or PC test is not effective in the sense that, even if performed correctly, it will not catch the original error.

\begin{tabular}{|c|c|c|c|c|c|c|}
\hline $\begin{array}{l}\text { Case } \\
\& \text { RFs }\end{array}$ & & $\begin{array}{l}\text { of } \\
\text { npo- } \\
\text { ats }\end{array}$ & $\begin{array}{l}\text { One } \\
\text { compo- } \\
\text { nent }\end{array}$ & $\begin{array}{l}\text { Paralle } \\
\text { (If } \mathrm{ZD}, \mathrm{s} \\
\mathrm{CD} \\
\text { (If not } \mathrm{ZD},\end{array}$ & $\begin{array}{l}\text { System } \\
\text { ee Note } 3 \text { ) } \\
\text { HD } \\
\text { see Note 4) }\end{array}$ & $\begin{array}{l}\text { Series System } \\
\text { ZD } \\
\text { (See Note 5) }\end{array}$ \\
\hline ase & I & $\begin{array}{l}1 \\
2 \\
3 \\
4 \\
5\end{array}$ & $3 E-2(5)$ & $\begin{array}{l}2 E-2(5) \\
2 E-2(5) \\
2 E-2(5) \\
2 E-2(5)\end{array}$ & $\begin{array}{l}1 E-2(6) \\
5 E-3(7) \\
3 E-3(7) \\
1 E-3(8)\end{array}$ & $\begin{array}{r}6 E-2(4) \\
9 E-2(3) \\
1.2 E-1(3) \\
1.5 E-1(2)\end{array}$ \\
\hline$(I x$ & $\begin{array}{l}\text { II } \\
.01)\end{array}$ & $\begin{array}{l}1 \\
2 \\
3 \\
4 \\
5\end{array}$ & $3 E-4(10)$ & $\begin{array}{l}2 E-4(10) \\
2 E-4(10) \\
2 E-4(10) \\
2 E-4(10)\end{array}$ & $\begin{array}{l}1 E-4(8) \\
5 E-5(9) \\
3 E-5(10) \\
1 E-5(11)\end{array}$ & $\begin{array}{r}6 E-4(5) \\
9 E-4(4) \\
1.2 E-3(4) \\
1.5 E-3(3)\end{array}$ \\
\hline $\begin{array}{l}\text { Case } \\
\text { ( I x }\end{array}$ & $\begin{array}{l}\text { III } \\
.1)\end{array}$ & $\begin{array}{l}1 \\
2 \\
3 \\
4 \\
5\end{array}$ & $3 E-3(10)$ & $\begin{array}{l}2 E-3(10) \\
2 E-3(10) \\
2 E-3(10) \\
2 E-3(10)\end{array}$ & $\begin{array}{l}1 E-3(11) \\
5 E-4(12) \\
3 E-4(13) \\
1 E-4(14)\end{array}$ & $\begin{array}{r}6 E-3(7) \\
9 E-3(6) \\
1.2 E-2(5) \\
1.5 E-2(4)\end{array}$ \\
\hline$\left(\begin{array}{ll}I & x\end{array}\right.$ & $\begin{array}{l}\mathrm{IV} \\
.1)\end{array}$ & $\begin{array}{l}1 \\
2 \\
3 \\
4 \\
5\end{array}$ & $3 E-3(10)$ & $\begin{array}{l}2 E-3(10) \\
2 E-3(10) \\
2 E-3(10) \\
2 E-3(10)\end{array}$ & $\begin{array}{l}1 E-3(11) \\
5 E-4(12) \\
3 E-4(13) \\
1 E-4(14)\end{array}$ & $\begin{array}{r}6 E-3(7) \\
9 E-3(6) \\
1.2 E-2(5) \\
1.5 E-2(4)\end{array}$ \\
\hline
\end{tabular}


Table 5-5 $\mathrm{F}_{\mathrm{T}} \mathrm{s}$ for Table 5-3 BHEPs, Modified for Multiple-Component Systems, Assuming Dependence Levels Determined by Using Guidelines in Table 5-4, and Including RFs (p3/3)

Note 7: In the last 5 cases below, the PM or PC test is effective i.e., if performed correctly, it will catch the original error.

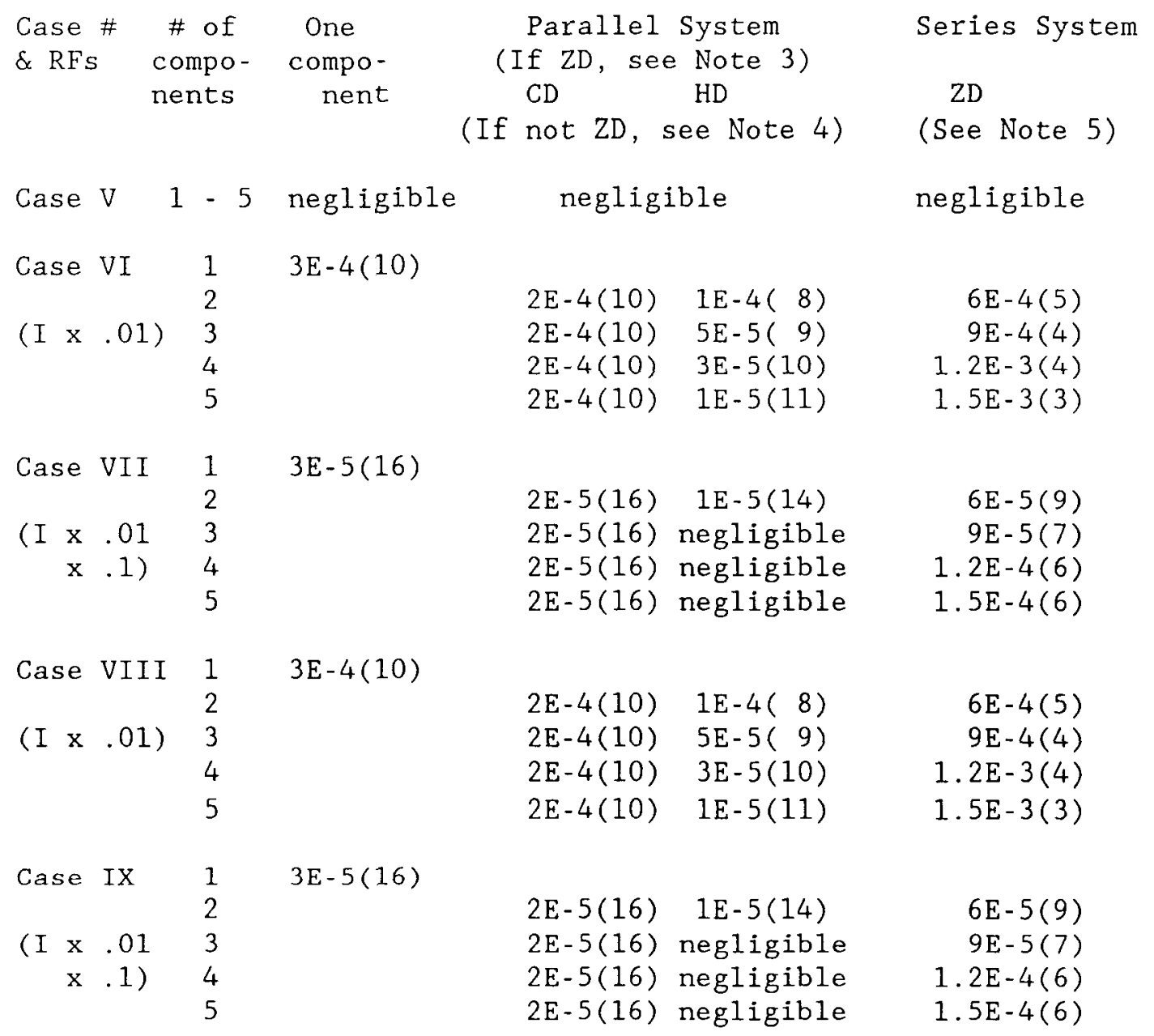

Note 8: In one ASEP PRA, additional conservatisms were employed. First, if the dependence level per Table 5-4 was not assessed as $2 D$, it was assessed as $C D$. That is, HD was not used. Second, for parallel systems, the basic HEP of .03 was employed for $C D$ rather than the .02 suggested in Note 4 above. 
PART III. ASEP HRA PROCEDURE FOR POST-ACCIDENT TASKS

This part consists of Chapter 6, "General Information for HRA Procedure for Post-Accident Tasks," Chapter 7, "ASEP Screening HRA for Post-Accident Tasks," and Chapter 8, "ASEP Nominal HRA for Post-Accident Tasks." Chapter 6 provides assumptions and other statements that pertain primarily to activities performed by operations personnel after annunciation of some abnormal event has occurred. Concepts are described that deal with knowledge-based behavior (i.e., diagnosis of the abnormal event) and the usual rule-based behavior associated with post-diagnosis actions. Chapter 7 presents the step-by-step procedure, models, and tables of estimated HEPs that one can use in performing the screening HRA for post-accident tasks. Chapter 8 presents the same kind of information for the nominal HRA. 
This chapter provides general information for the screening human reliability analysis (HRA) and the nominal HRA for post-accident tasks. To develop rules for the screening and nominal HRAs, modifications were made to the RMIEP screening HRA procedure [see Swain (1985a) or the section on "Screening Rules for the Human Reliability Analysis in the Risk Methods Integration and Evaluation Program" in Volume 5 of NUREG/CR-4832 (Payne et al, 1987)], which in turn was based primarily on modifications of NUREG/CR-1278. See Appendix A for more information on the relationship of the RMIEP screening HRA procedure to the ASEP HRA procedure for post-accident tasks. The rules for estimating human error probabilities (HEPs) and uncertainty bounds (UCBs) for ASEP screening HRAs and nominal HRAs for post-accident tasks are found in, respectively, Chapter 7 and Chapter 8.

To develop an HRA procedure for post-accident tasks, a simplified model of human behavior for these tasks was devised. The model makes some simplifying assumptions about diagnosis behavior, and clarifies some of the rules in NUREG/CR-1278 which were only implicit. In addition, rules for allowing credit for the new symptom-oriented emergency operating procedures (EOPs) are provided. This chapter provides a background for the general models found in Chapters 7 and 8 .

\section{Definitions of Terms}

Before proceeding further, it is suggested that the reader consult the prefatory pages to review the list of abbreviations and definitions of the following terms, which pertain to post-accident tasks. Some of these terms are also defined in the text which follows.

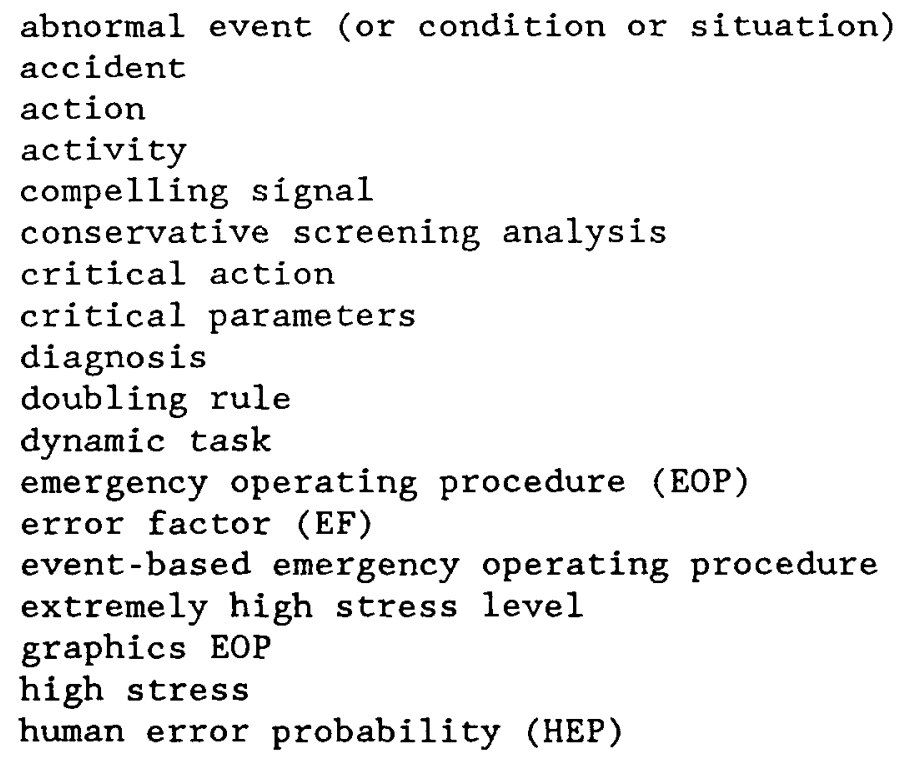




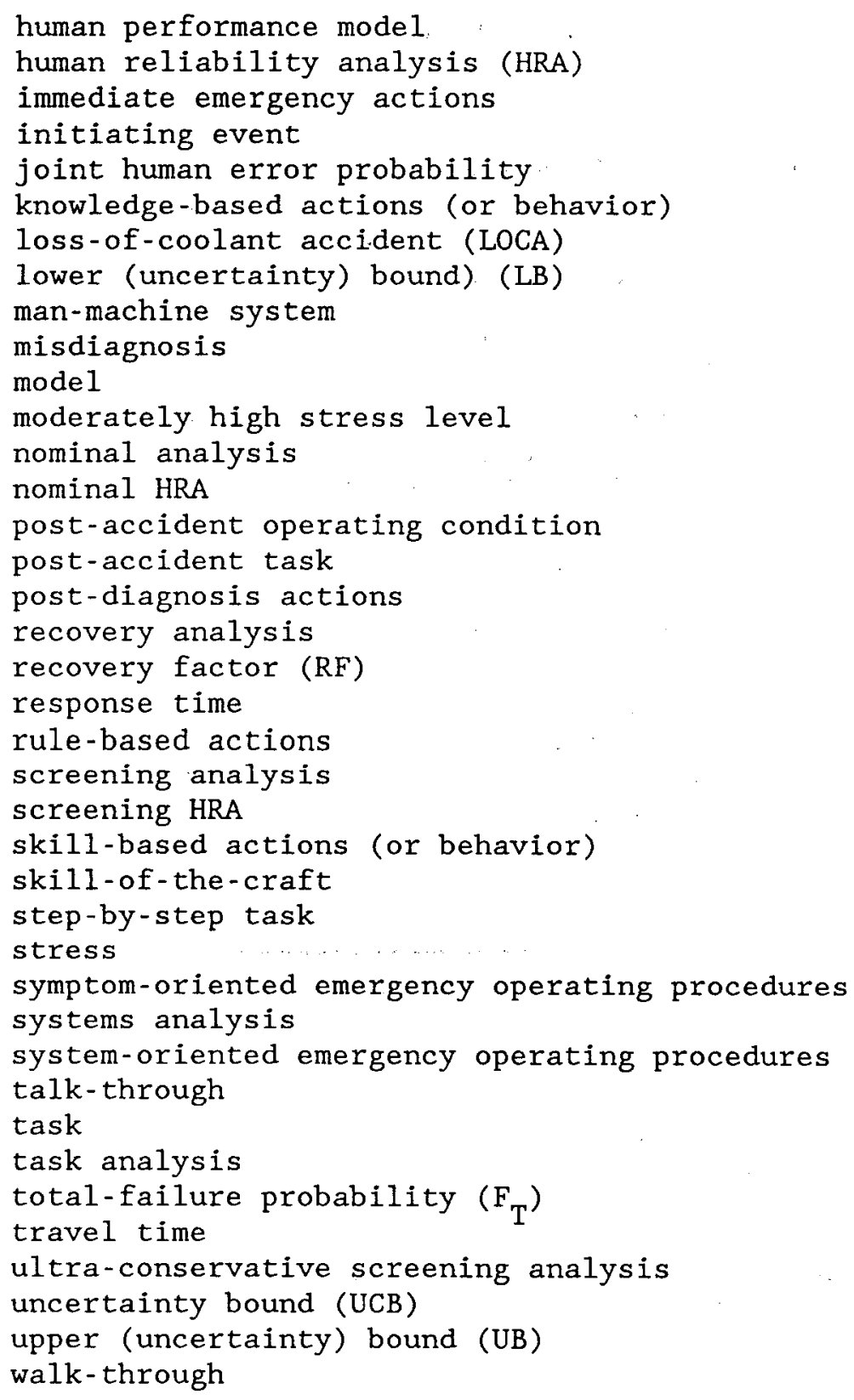

\section{Post-Accident Tasks of Interest}

Post-accident tasks are divided into diagnosis tasks and post-diagnosis tasks, both of which are intended to maintain or ensure reactor protection once some abnormal event. has occurred. Diagnosis refers to the probability of a correct diagnosis within the time required to permit the carrying out of the required post-diagnosis actions. Diagnosis is defined as the attributing of the most likely cause(s) of an abnormal event to the level required to identify those systems or components whose status can be changed to reduce or eliminate the 
problem. In the context of the new symptom-oriented EOPs, diagnosis does not necessarily require that some name be attached to the abnormal event, e.g., small loss-of-coolant accident (LOCA).

In short, diagnosis merely means figuring out what to do when an abnormal event has been recognized. Diagnosis involves knowledge-based behavior, as defined in Table 2-1. Post-diagnosis actions are those taken which logically follow a correct diagnosis of the abnormal event. Post-diagnosis actions involve rule-based or skill-based behavior (Table 2-1). Diagnosis and related words (cognition, interpret, decide, judgment, etc.) are often used differently by different people, and dictionary definitions are not always helpful, as indicated in the second column in Table 6-1. The ASEP HRA Procedure uses the definitions in the third column of this table. The human activity does not always follow the sequence shown in this column. Instead, there are many possible interactions and shortcuts in responding to an abnormal event, as shown in Figure 6-1.

The specific abnormal events to be analyzed in HRAs in the ASEP probabilistic risk assessments (PRAs) were designated by systems analysts. They included such events as station blackouts, anticipated transients without scram (ATWS), loss-of-coolant accidents (LOCAs), loss of AC or DC bus, and loss of component cooling water.

The identification of the related human behaviors and potential errors in each of the abnormal events analyzed in a PRA is accomplished by a team of systems analysts, human reliability analysts, appropriate plant personnel (especially licensed reactor operators and simulator instructors), and others as needed. The team uses the typical task analysis methods (interviews, observations, simulator exercises, etc.) described in Chapter 4 of NUREG/CR-1278 and in NUREG/CR-2254. As shown in Figure 6-2, an HRA of post-accident tasks typically starts with the annunciation (or other compelling signal) of an abnormal event: For purposes of PRA, there are only two success paths. One of these success paths (the top path in the figure) requires a correct diagnosis to be made within time constraints, as defined later, and the appropriate postdiagnosis actions (primarily rule-based behavior) to be correctly performed within their time constraints (again, as defined later). The other success path (which has rarely been considered in published PRAs, including the ASEP PRAs) allows a misdiagnosis, but assumes a successful recovery from the misdiagnosis, followed by the correct performance of the post-diagnosis actions, again all within the allowable time constraints. 
Table 6-1 Definitions of Cognition-Related Terms and Usage in the Handbook of Human Reliability Analysis ( $1 / 2$ )

(Copy of Table 12-1 from NUREG/CR-1278)

\begin{tabular}{|c|c|c|}
\hline Term & Dictionary Definition* & Handbook Usage \\
\hline Cognition & $\begin{array}{l}\text { the act or process of } \\
\text { knowing, including both } \\
\text { awareness and judgment }\end{array}$ & $\begin{array}{l}\text { restricted to those aspects of } \\
\text { behavior involved in diagnosis } \\
\text { of abnormal events }\end{array}$ \\
\hline Judgment & $\begin{array}{l}\text { the process of forming an } \\
\text { opinion or evaluation by } \\
\text { discerning and comparing }\end{array}$ & $\begin{array}{l}\text { not used in our models--too } \\
\text { imprecise; used only in the } \\
\text { context of expert estimation }\end{array}$ \\
\hline Perceive & $\begin{array}{l}\text { to attain awareness or } \\
\text { understanding; to become } \\
\text { aware through the senses }\end{array}$ & $\begin{array}{l}\text { used in the very narrow sense of } \\
\text { "awareness" without the further } \\
\text { meaning of "understanding, "e.g., } \\
\text { "some annunciator tiles over } \\
\text { there are blinking" }\end{array}$ \\
\hline Discriminate & $\begin{array}{l}\text { to mark or perceive the } \\
\text { distinguishing or peculiar } \\
\text { features of; } \\
\text { to distinguish one like } \\
\text { object from another }\end{array}$ & $\begin{array}{l}\text { distinguishing one signal (or a } \\
\text { set of signals) from another, } \\
\text { e.g., "the coolant level in } \\
\text { Tank A is } 37 \text { feet, " or if there } \\
\text { are limit marks on the meter, } \\
\text { "the coolant level is out of } \\
\text { limits" (in the latter case, some } \\
\text { interpretation is done for the } \\
\text { operator by the design of the } \\
\text { display) }\end{array}$ \\
\hline Interpret & $\begin{array}{l}\text { to conceive in the } 1 \text { ight } \\
\text { of individual belief, } \\
\text { judgment, or circumstance }\end{array}$ & $\begin{array}{l}\text { the assignment of a meaning to } \\
\text { the pattern of signals (or stimu- } \\
\text { li) that was discriminated, e.g., } \\
\text { "the coolant level in Tank A is } \\
\text { low, which means that the make-up } \\
\text { pump is not running, or there is } \\
\text { a leak somewhere, or the indica- } \\
\text { tor is out of order"; if there is } \\
\text { only one possible cause for the } \\
\text { observed signal, the interpre- } \\
\text { tation is equivalent to diagnosis }\end{array}$ \\
\hline
\end{tabular}

*Webster (1975) 
Table 6-1 Definitions of Cognition-Related Terms and Usage

in the Handbook of Human Reliability Analysis ( $2 / 2$ )

(Copy of Table 12-1 from NUREG/CR-1278)

\begin{tabular}{|c|c|c|}
\hline Term & Dictionary Definition* & Handbook Usage \\
\hline Diagnosis & $\begin{array}{l}\text { a statement or conclusion } \\
\text { concerning the nature or } \\
\text { cause of some phenomenon }\end{array}$ & $\begin{array}{l}\text { the attributing of the most like- } \\
\text { ly cause(s) of the abnormal event } \\
\text { to the level required to identify } \\
\text { those systems or components whose } \\
\text { status can be changed to reduce } \\
\text { changed to reduce or eliminate } \\
\text { the problem; diagnosis includes } \\
\text { interpretation and (when } \\
\text { necessary) decision-making }\end{array}$ \\
\hline Decide & $\begin{array}{l}\text { to make a choice or } \\
\text { judgment }\end{array}$ & $\begin{array}{l}\text { "decision-making" used instead of } \\
\text { "deciding" }\end{array}$ \\
\hline $\begin{array}{l}\text { Decision- } \\
\text { making }\end{array}$ & & $\begin{array}{l}\text { (1) decision-making as part of } \\
\text { diagnosis: the act of } \\
\text { choosing between alternative } \\
\text { diagnoses, e.g., to settle on } \\
\text { the most probable cause of } \\
\text { the pattern of stimuli asso- } \\
\text { ciated with an abnormal event } \\
\text { (2) post-diagnosis decision- } \\
\text { making: the act of choosing } \\
\text { which acts to carry out after } \\
\text { a diagnosis has been made; } \\
\text { in most cases, these actions } \\
\text { are prescribed by rules or } \\
\text { procedures, and decision- } \\
\text { making is not required }\end{array}$ \\
\hline Action & $\begin{array}{l}\text { a thing accomplished } \\
\text { usually over a period of } \\
\text { time, in stages, or } \\
\text { with the possibility of } \\
\text { repetition }\end{array}$ & $\begin{array}{l}\text { carrying out one or more } \\
\text { activities (e.g., steps or } \\
\text { tasks) indicated by diagnosis, } \\
\text { operating rules, or } \\
\text { written procedures }\end{array}$ \\
\hline
\end{tabular}

*Webster (1975) 


\section{COGNITION-RELATED TERMS}

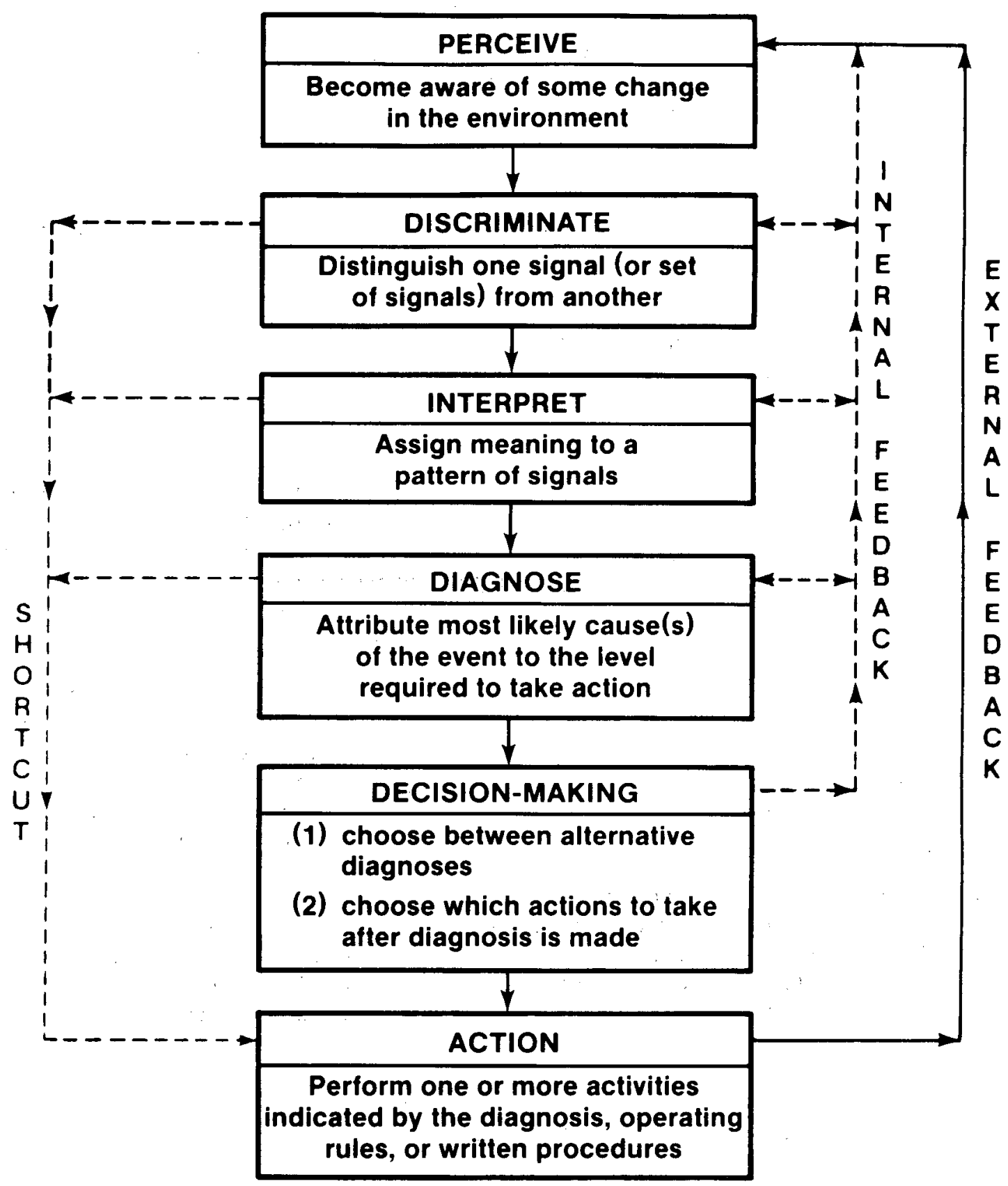

Figure 6-1 Interaction of Cognition-Related Terms

(Copy of Figure 1 from Swain and Weston, 1987) 


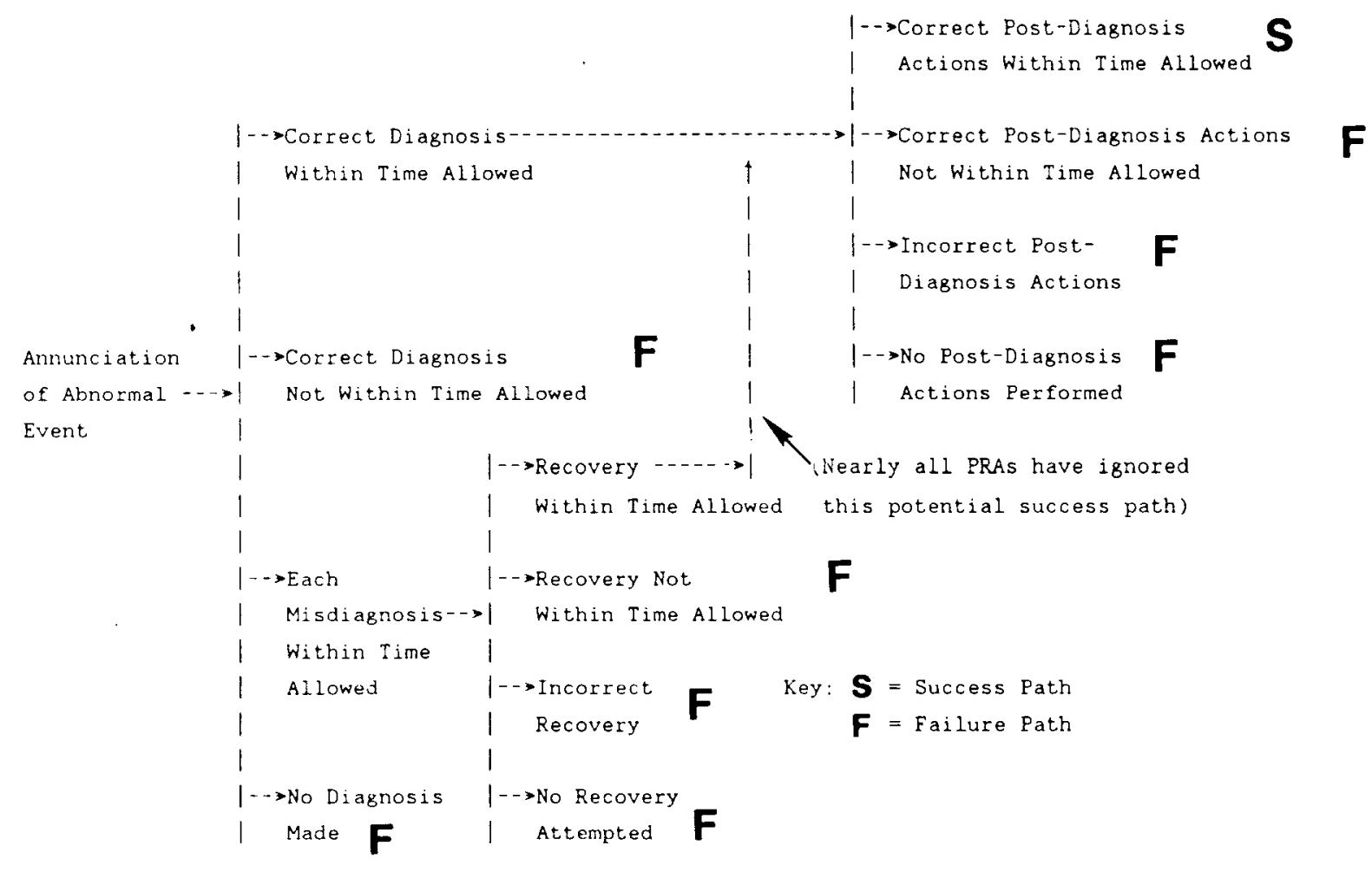

Figure 6-2 Success and Failure Paths Following an Abnormal Event

(Copy of Figure 2 from Swain and Weston, 1987)

There are eleven failure paths in Figure 6-2, as indicated by the left-most five $F$ terms at the end of single paths, and the three right-most $F$ terms, each of which can be reached by two different paths through the event tree. Note that in these paths, diagnosis, recovery actions, and post-diagnosis actions are considered to be failures because they were not performed on a timely basis, or they were performed incorrectly, or no action at all was taken. Often it is desirable to estimate probabilities of specific incorrect actions, as they may result in worsening the abnormal situation. The ASEP HRA Procedure does not address this particular HRA problem; see p 21-14 to 21-24 of NUREG/CR-1278 for an example analysis of this difficult area.

Some Simplifications in the HRA Procedure for Post-Accident Tasks

In keeping with a primary goal of the ASEP, several important simplifications have been made. One of the major simplifications is to ignore the whole area of specific misdiagnoses. Instead, it is conservatively assumed that any failure to correctly diagnose an abnormal event within the allowable time will result in a core damage accident. No analysis is made of the possible kinds 
of erroneous diagnoses (i.e., misdiagnoses) that might be made for any abnormal event. For a suggested new approach to this problem, see Swain and Weston (1987).

Another simplification is to segment the estimated total time available for coping with an abnormal event into artificially independent parts. For example, consider the top success path in Figure 6-2. A total allowable time for coping with an abnormal event is specified by systems analysts and is divided into an allowable diagnosis time and an allowable post-diagnosis time. This HRA approach involves the estimation of two separate time-dependent probabilities: the probability of performing a correct diagnosis within its allowable time, and the probability of performing the correct post-diagnosis actions within its allowable time. Then, the product of these two probabilities is taken as the probability that a correct diagnosis will be made and that the correct post-diagnosis actions will be completed within the total allowable time. This is not literally true because different combinations of time-dependent probabilities for the two time periods are not considered. It appears that the simplification suggested can result in very conservative estimates of the total failure probabilities of coping successfully with abnormal events. In the absence of data which would permit full consideration of time dependencies, this simplification is considered to be acceptable.

Another simplification is to assume that there is only one, correct sequence of activities in coping with any specified post-accident sequence. In the ASEP PRAs, the correct sequence was selected from the EOPs for an abnormal event. This restriction does not keep the analyst from analyzing several different sequences of activities for an abnormal event, as defined by different postaccident assumptions. For example, in an ATWS, one sequence might assume that boron injection is available, and a different sequence might assume that this engineered safety feature (ESF) is unavailable. In such a case, we really have two different abnormal events, and the human behaviors involved will have important differences. A different HRA is required for each different postaccident sequence.

Other simplifications specific to screening and nominal HRAs for post-accident tasks are stated in Chapters 7 and 8.

\section{Time Dependencies Between Diagnosis and Post-Diagnosis Tasks}

The approach used in the ASEP HRA Procedure to estimating HEPs for post-accident tasks is taken from Chapter 12 in NUREG/CR-1278. First, one ascertains by measurement or estimation the time that is required to perform the necessary rule-based actions once the control room personnel understand what needs to be done. In short, these are the actions following a correct diagnosis. Once a time measurement or estimation has been made for these post-diagnosis actions, this time is subtracted from the total man/machine system response time that systems analysts have estimated is allowable for acceptable system response to the abnormal event. The time that is left after this subtraction is the time permitted for diagnosis. The problem, then, is to estimate the probability of a correct diagnosis within that allowable time. 
Figure 6-3 diagrams the above time relationships, and the following steps provide additional descriptive information. The procedure in chapters 7 and 8 is based on this approach, and examples in both chapters illustrate the use of Figure 6-3.
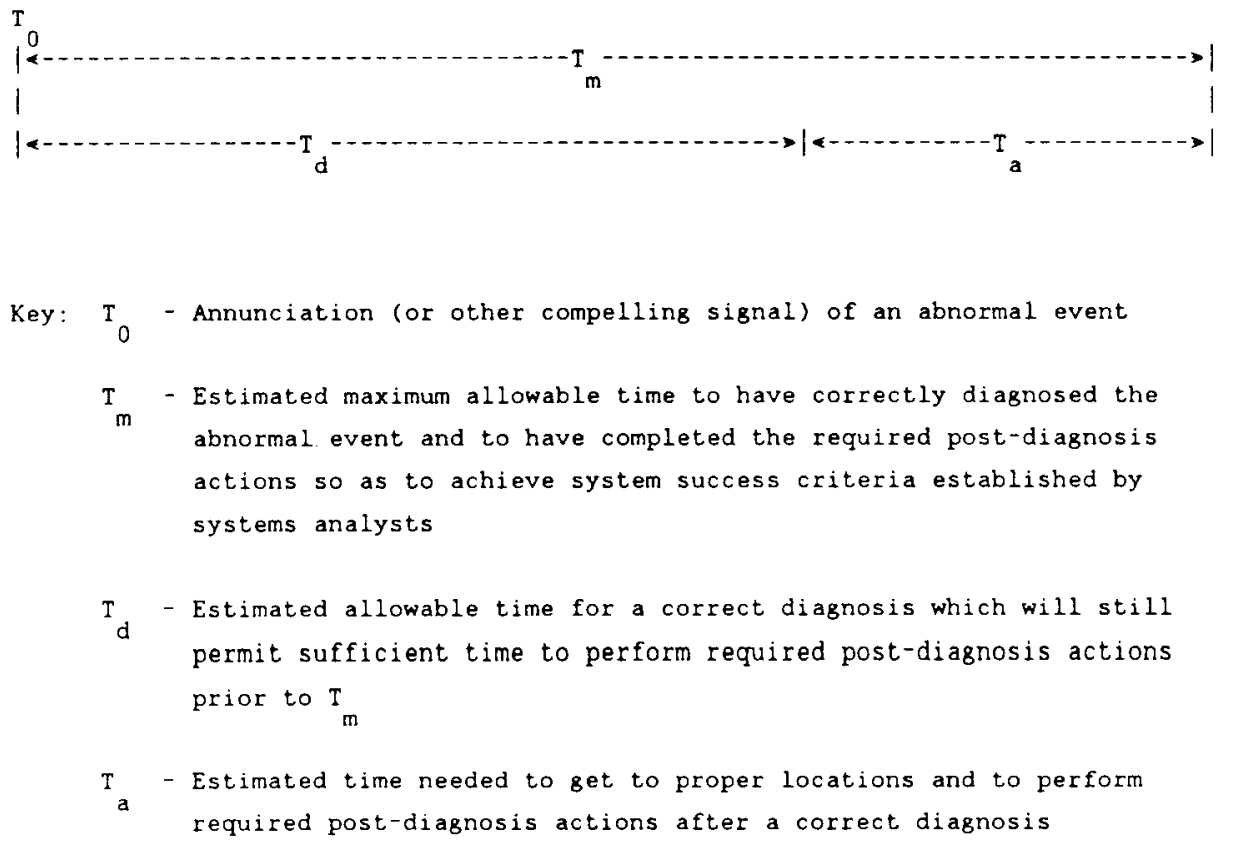

Figure 6-3 Time Relationships between Annunciation (or Other Compelling Signal) of an Abnormal Event, a Correct Diagnosis of the Event, and Performing the Required Post-Diagnosis Actions After a Correct Diagnosis

(Copy of Figure 3 from Swain and Weston, 1987)

\section{Procedure}

1. Estimate $T_{m}$, the maximum allowable time to have diagnosed the abnormal event and have completed the necessary rule-based (or skill-based) actions. $\mathrm{T}_{\mathrm{m}}$ is determined by systems analysts, based on an analysis of plant operating characteristics. 
2. Identify the rule-based and skill-based actions that control room personnel would be likely to take following the correct diagnosis of each abnormal event in the accident sequence of interest. For the ASEP HRA Procedure, the PRA team selects one set of post-diagnosis actions as the most likely, and, for simplicity, assumes that this is the only plausible set.

3. Estimate $T$, the time needed to get to the proper locations inside or outside of the control room and to perform the required actions following correct diagnosis of an abnormal event (see Step 2). $T$ is designated as time for "post-diagnosis actions." The HRA analyst pertorms this estimation using appropriate plant personnel as subjects. Actual measurements are used wherever possible, in the simulator for control room activities, and by using walk-throughs for activities performed outside the control room. Only as a last resort should opinions be substituted for time measurements, as data show that time estimates from judgments are quite variable, and usually underestimate the time required for tasks (see $p$ 6-11 in NUREG/CR-1278).

To reduce the number of measurements needed, actions can first be grouped, based upon similarity of location and type of action. For example, all actions requiring movement of a simple switch and located on the same control-room panel can be grouped. Then, measurements need be taken on only one action per group, since similar times would be expected for all actions within a group.

In practice, estimates of $T_{\text {a }}$ tend to be conservative, that is, the es timates do not represent median response times. Instead, at least as practiced by the author, they represent times which should be quite sufficient to allow for error-free performance of the actions in question.

4. Calculate $\mathrm{T}_{\mathrm{d}}=\mathrm{T}_{\mathrm{m}}-\mathrm{T}_{\mathrm{a}}$, the estimated maximum allowable time permitted for correct diagnosis so as to allow sufficient time to perform the required post-diagnosis actions prior to $\mathrm{T}_{\mathrm{m}}$.

5. Using the procedure in Chapter 7 or 8 , estimate the probability of failing to correctly perform within $\mathrm{T}_{\mathrm{a}}$ the required post-diagnosis actions identified in step 3 .

6. Using the procedure in Chapter 7 or 8 , estimate the probability that a control room team will fail to diagnose correctly each abnormal event of interest within its time $\mathrm{T}_{\mathrm{d}}$, the maximum time allowable for the crew to figure out what must be done so that they still have time to perform the necessary post-diagnosis actions by the end of $\mathrm{T}_{\mathrm{m}}$. 
CHAPTER 7 ASEP SCREENING HRA FOR POST-ACCIDENT TASKS

\section{General Information}

Unlike the HRA screening procedure for pre-accident tasks (see Chapter 4), the screening procedure for post-accident tasks does not provide upper uncertainty bounds as a basis for an ultra-conservative screening analysis. It is judged that the screening analysis presented herein is already sufficiently conservative. The screening procedure in this chapter is very similar to the RMIEP HRA Screening Procedure for post-accident tasks [see Swain (1985a) or the section on "Screening Rules for the Human Reliability Analysis in the Risk Methods Integration and Evaluation Program" in Volume 5 of NUREG/CR-4832 (Payne et al, 1987)], with some exceptions to increase the conservatism of the ASEP screening HRA. See Appendix A for more detail on the relationship of RMIEP HRA Screening Procedure to the ASEP HRA screening HRA for post-accident tasks.

The following tables and figures present the background material for the ASEP screening HRA for post-accident tasks. Table 2-1 defines skill-, rule-, and knowledge-based behavior. Table 6-1 and Figure 6-1 define and diagram the interrelationships of diagnosis and other cognition-related terms. Figure 6-3 shows the required time relationships among $\mathrm{T}_{0}, \mathrm{~T}_{\mathrm{m}}, \mathrm{T}_{\mathrm{a}}$, and $\mathrm{T}_{\mathrm{d}}$.

With the exception of the HEPs in Table 7-3 for post-diagnosis actions, the error factors (EFs) for the estimated screening HEPs are included in the tables taken from NUREG/CR-1278. The EFs 1isted in Table 7-3 were selected to provide conservatism, especially to avoid unduly low uncertainty bounds.

\section{The Procedure}

The detailed procedure for the screening HRA for post-accident tasks is presented in Tables 7-1, 7-2, and 7-3 and Figure 7-1. Table 7-1 provides the basic procedure, with references to other tables (or figures), as appropriate. Table 7-2 and and Figure 7-1, the screening diagnosis model, are taken from NUREG/CR-1278. Table 7-3 presents the rules for estimating HEPs for the postdiagnosis tasks. A hypothetical example at the end of this chapter shows how the ASEP screening HRA procedure might be used for post-accident tasks. 
Table 7-1 Procedure for Screening HRA of Post-Accident Tasks (p1/4)

1. Review the definitions and concepts in Table 2-1 (defining skill-, rule-, and knowledge-based behavior) and Table 6-1 and Figure 6-1 (defining and diagramming diagnosis and other cognition-related terms), and Figure 6-3 (showing the required time relationships of $\mathrm{T}_{0}, \mathrm{~T}_{\mathrm{m}}, \mathrm{T}_{\mathrm{a}}$, and $\mathrm{T}_{\mathrm{d}}$ ).

2. For the following cases, assess HEP $=1.0$ for the entire HRA for the abnormal event in question; no further HRA is required:

a. Critical activities must be performed outside of the control room area.

b. Critical skill-based or rule-based post-diagnosis actions are not described in written procedures. (Details of skill-based actions are not required to be written if they can be classified as "skill-of-thecraft" - see the prefatory section "Definitions of Technical Terms.") This assessment is used even though it may be required for personnel to have memorized these actions. Instead, they would likely refer to the written procedures at a later time during the usual checking to see that all immediate emergency actions had been performed correctly. Lack of written procedures is considered indicative of inadequate quality assurance, and is the justification for this assessment. However, it is not expected that the diagnosis aspect of every abnormal event would be treated in detail in the written procedures; the rules in this table for assessing diagnosis HEPs are treated separately.

c. The required instrumentation fails to support diagnosis or post-diagnosis behavior, or the instrumentation is inaccurate (i.e., misleading).

3. Using systems analysis methods, and referring to Figure 6-3, estimate $T_{m}$, the maximum allowable time to have correctly diagnosed an abnormal event and to have completed the necessary human actions following $\mathrm{T}_{0}$, the annunciation (or other compelling signal) of an abnormal event. For definitions of diagnosis and related terms, see Table 6-1 and Figure 6-1.

4. Identify the actions required to successfully cope with the abnormal event, once a correct diagnosis has been made.

5. For post-diagnosis actions to be performed in the control room area, estimate travel time and manipulation time, as follows:

a. If there is a requirement to use written procedures, i.e., the human actions to be performed cannot be assumed to be committed to memory, assess a 5-minute delay, after correct diagnosis, before the required post-diagnosis actions will be initiated. 
Table 7-1 Procedure for Screening HRA of Post-Accident Tasks (p2/4)

b. Assess 1 minute as the required travel and manipulation time combined for each control room (CR) control action taken on the primary operating panels which are normally in visual access of the $C R$ operator. An example is activation of the manual trip button.

c. For required control actions in the control room area, but on other than the primary $C R$ operating panels, assess 2 minutes as the required travel and manipulation time for each such control action.

d. For screening purposes, do not consider possible assignments of personnel to monitor particular panels.

6. Sum the estimated times from step 5 to calculate $T$, the time needed to get to a particular location plus the time needed to perform required actions once a diagnosis of an abnormal event has been made.

7. Calculate $T_{d}=T_{m}-T_{a}$, which is the allowable time for a diagnosis which permits the performance of the required actions within the total allowable time, $T_{m}$. See Figure 6-3.

8. Using $T_{d}$, select the appropriate diagnosis HEP from Figure 7-1 or Table 72. This screening diagnosis HEP is a joint HEP representing the performance of the entire control room crew. Adjust the HEP using the rules stated below.

a. This diagnosis HEP is considered the probability of misdiagnosis which will result in a core damage accident.

b. For the case of more than one abnormal event occurring closely in time (i.e., within 10 minutes), use Table 7-2 to estimate the diagnosis HEP for the second or subsequent simultaneously occurring abnormal event. Use the guidelines below in judging whether to assess more than one abnormal event in an accident sequence being evaluated.

1) If it is not possible to obtain the information in items 2) - 5) below, make the most conservative assumption. 
Table 7-1 Procedure for Screening HRA of Post-Accident Tasks ( $\mathrm{p} 3 / 4$ )

2) If the emergency operating procedure (EOP) appropriate to the accident sequence does not specifically describe any additional events being evaluated, including any change in first-event status being evaluated, reapply the screening diagnosis model. In deciding whether to reapply this model, it is not necessary that the EOP specifically name the additional abnormal events or changes in status; it must, however, lead the operators to cope successfully with these conditions if they correctly use the EOP. That is, the EOP must enable the operators to figure out what to do (or be led to the correct actions) in coping with the accident sequence details being evaluated. If the EOP does provide this guidance, do not assess the need for a second or subsequent diagnosis.

3) If the second or subsequent abnormal event occurs "closely in time" with the first event, as defined in the first footnote to Table 7-2, employ the second column in the table. If the second or subsequent abnormal event occurs later, and it can be judged that the control room personnel are no longer actively engaged in diagnosing and/or planning the responses to cope with the first event, use the first column for such additional events.

4) Note that for any third or subsequent abnormal event assessed as occurring "closely in time," as defined in Table 7-2, a diagnosis HEP of 1.0 is assessed.

5) Note that in Table 7-2 $\mathrm{T}_{0}$ refers to a compelling signal of the initiation of any abnormal event and that a probability of 1.0 is assumed for observing that there is some abnormal situation. If there are more than two competing compelling signals (e.g. annunciators), assess a diagnosis HEP of 1.0 .

c. For the diagnosis HEP for reactor vessel/containment critical parameters which operating personnel must commit to memory, use the lower bound values in Figure 7-1 or Table 7-2 only if the recognition of these parameters can be classified as skill-based behavior per Table $2-1$; otherwise, use the nominal values.

As an example of reactor vessel/containment critical parameters which all the CR reactor operators commit to memory, the four critical parameters at LaSalle Boiling Water Reactor (BWR) nuclear power plant (NPP) are:

- Check reactor power level. It must not exceed $118 \%$.

- Check the water level in the core. It must not be below 12.5 inches above instrument zero.

- Check reactor pressure. It must not be over 1046 psi.

- Check containment temperature and pressure. Temperature must not be over 110 degrees and pressure must not be over 1.69 psi. 
Table 7-1 Procedure for Screening HRA of Post-Accident Tasks ( $\mathrm{p} 4 / 4)$

d. If it can be determined that all control room operators are trained to quickly initiate a manual scram signal with the SCRAM switches when the annunciation of an automatic scram has occurred, or when an immediate indication of a failure to scram has occurred, assess a negligible probability of a diagnosis error, and instead assess only the failure to perform the correct switching action given that a correct diagnosis has occurred. Assume that any correct activation of the SCRAM switches will occur within one minute of the annunciation of a call for an automatic scram. In the case of BWRs, the same argument applies to manual activation of the switch which precludes early closure of the Main Steam Isolation Valves (MSIVs) due to low steam pressure if the same signals and training for manual activation of the MSIV preclude switch (often called MODE switch) can be assumed. However, in the latter case, assume complete dependence (CD) between the activation of the manual SCRAM switch and the MSIV preclude switch. The above assessment is equivalent to assigning an HEP of .01 (from Table 7-3, item 5) for failure to correctly perform the manual switching action (or, for BWRs, actions).

9. Select the appropriate $\operatorname{HEP}(s)$ for post-diagnosis action(s) from Table 7-3.

10. Calculate the estimated total-failure probability, $F_{T}$, by adding the diagnosis HEP (Step 8) to the HEP(s) for carrying out the required postdiagnosis action(s) (Step 9). If this calculation results in a totalfailure probability greater than 1.0 , use 1.0 .

11. Enter the $F_{T} s$ in the appropriate system fault trees or system event trees, paying special attention that the dependence effects identified for human actions are preserved in the way the $F_{T} s$ are used. See Chapter 5 of NUREG/CR-1278 for guidelines. 


\section{NOMINAL DIAGNOSIS MODEL}

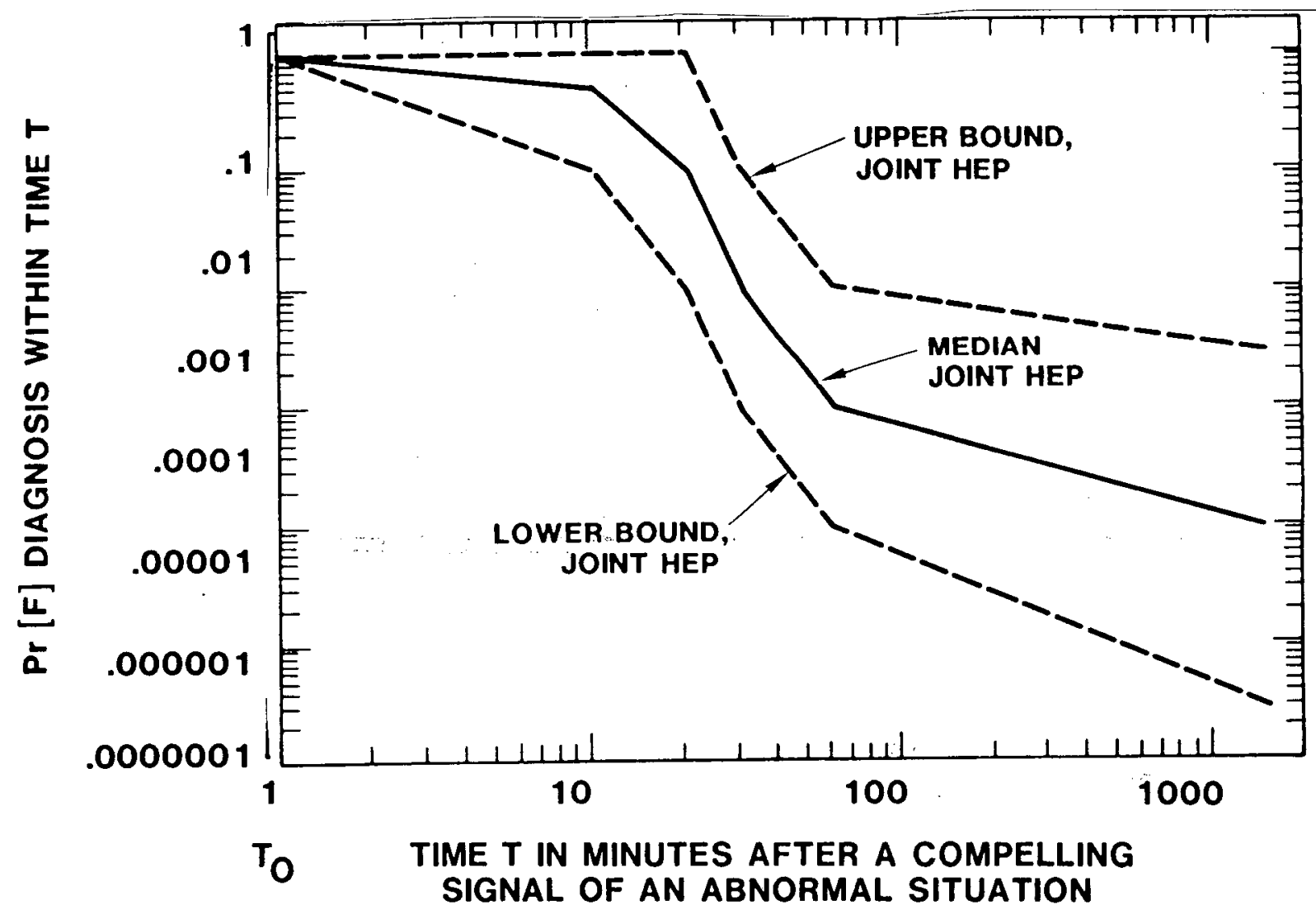

Figure 7-1 Initial-Screening Model of Estimated HEPs and UCBs for Diagnosis Within Time $\mathrm{T}$ of One Abnormal Event by Control Room Personnel

(Revised copy of Figure 12-3 from NUREG/CR-1278. The revision corrects the labeling of the ordinate in the figure so that the "1" occurs where the three lines in the figure meet at the ordinate, as shown in the above figure.) 
Table 7-2 Initial-Screening Model of Estimated HEPs and EFs for Diagnosis Within Time $\mathrm{T}$ by Control Room Personnel of Abnormal Events Annunciated Closely in Time*

(Copy of Table 20-1 from NUREG/CR-1278 with appropriate changes to figure number)

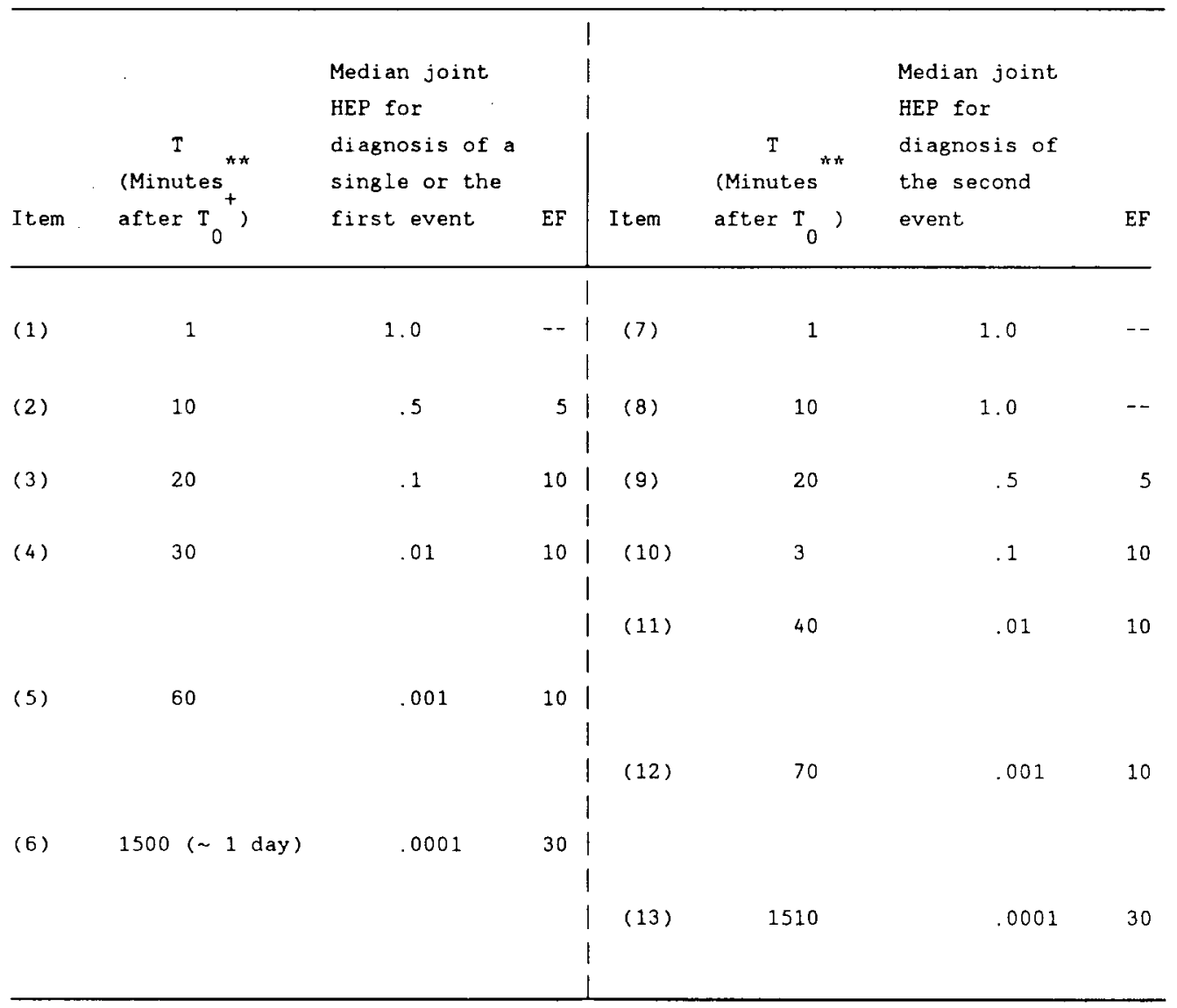

"Closely in time" refers to cases in which the annunciation of the second abnormal event occurs while $C R$ personnel are still actively engaged in diagnosing and/or planning responses to cope with the first event. This is situation-specific, but for the initial analysis, use "within 10 minutes" as a working definition of "closely in time."

Note that this model pertains to the CR crew rather than to one individual.

** For points between the times shown, use the medians and EFs from Figure 7-1 for the first event, and interpolate between the tabled values for the second event.

${ }^{+} \mathrm{T}$ is a compelling signal of an abnormal situation and is usually taken as a pattern of annunciators. A probability of 1.0 is assumed for observing that there is some abnormal situation.

$++$

Assign HEP $=1.0$ for the diagnosis of the third and subsequent abnormal events annunciated closely in time. 
Table 7-3 Assessment of Screening HEPs for Post-Accident Post-Diagnosis Actions

Item $\underline{\text { HEP }} \quad \underline{\mathrm{EF}}$ Action*

(1) 1.0 - Perform a required action outside of control room.

(2) 1.0 - Perform a critical skill-based or rule-based action correctly when no written procedures are available. (Details of skillbased actions are not required to be written if they can be classified as "skill-of-the-craft"**.) This assessment is used even though it may be required for personnel to have memorized these actions. Instead, they would likely refer to the written procedures at a later time during the usual checking to see that all immediate emergency actions had been performed correctly. (See Table 2-1 for definitions.)

HEPs (3) and (4) are for performing a critical procedural action correctly under "moderately high stress" $* *$ or "extremely high stress"**. For screening, at least moderately high stress is assessed for all post-accident conditions. Also for screening, do not give any credit for recovery factors, e.g., a second person. Assume that only one person is available to perform the postdiagnosis tasks, and no one is available to check his accuracy.
(3) $.05 \quad 5$ Perform a critical procedural action correctly under moderately high stress.
(4) $.25 \quad 5$ Perform a critical procedural action correctly under extremely high stress.

(5) .01

5 Perform a post-diagnosis immediate emergency action for the reactor vessel/containment critical parameters, when (a) it can be judged to have been committed to memory, (b) it can be classified as skill-based actions per Table 2-1, and (c) there is a backup written procedure.

*The HEPs are for independent actions or independent sets of actions in which the actions making up the set can be judged to be completely dependent. Other levels of dependence among actions can be assessed by the analyst, using one or more methods for assessing dependence described in Chapter 10 of NUREG/CR-1278.

$* *$ See the prefatory section "Definitions of Technical Terms" for definitions of these frequently misunderstood terms. 


\section{A Hypothetical Example of a Post-Accident HRA}

The following hypothetical simple example illustrates the use of part of the screening procedure (Table 7-1), including the use of the method for estimating the appropriate time relationships between diagnosis and post-diagnosis actions (Figure 6-3), the screening diagnosis model (Figure 7-1 and Table 72 ), and the screening rules for post-accident, post-diagnosis actions (Table $7-3)$.

Assume that the human responses to a particular abnormal event are to be analyzed, using a screening HRA. It has been determined that there is only one critical post-diagnosis action of interest, which requires the use of a written procedure, and this procedure is available. This critical action requires the manipulation of a switch in the control room, but the switch is not on the primary panel. In the accident sequence being evaluated, it is assumed that the required instrumentation and related displays work properly.

Per step 3 in Table 7-1, the systems analysts have estimated $T_{\mathrm{m}}$ as 30 minutes. Reference to Figure 6-3 (as cited in step 3 of Table 7-1) shows that the control room crew must have completed all appropriate actions in the time interval between the annunciation of the event $\left(T_{0}\right)$ and $T_{m}$, in this case, within 30 minutes. Reference to steps 5 and 6 in Table 7-1 indicates that $\mathrm{T}$ should be assessed as 7 minutes ( 5 minutes per step 5 .a plus 2 minutes per step 5.c). $\mathrm{T}_{\mathrm{d}}$ is calculated per step 7 , as $30-7=23$ minutes.

Step 8 in Table 7-1 refers the analyst to Figure 7-1 or to Table 7-2 to obtain the estimated diagnosis HEP for the calculated 23 minutes that are available for the diagnosis. Normally, one would prefer to use Table 7-2, as it is easier to read correctly. However in this case, the second footnote to that table refers the analyst to Figure 7-1, as the table has no value for 23 minutes. Using Figure 7-1, the diagnosis HEP for 23 minutes is about .06 . Assume that none of the rules for adjusting this estimate (as stated under step 8) are relevant to the problem.

Step 9 in Table 7-1 refers the analyst to Table 7-3 to estimate the HEP for the one critical post-diagnosis action. Assume that a moderately high stress level is assessed. Therefore item 3 in the table results in an assessment of .05 as the HEP for the critical post-diagnosis action of interest.

Figure 7-2 shows an HRA Event Tree as one method of analysis per step 10 in Table 7-1. (For a description of this type of event tree, see "HRA event tree" in the prefatory section on "Definitions of Technical Terms.) In the figure, "F7-1, 23 min" refers to Figure 7-1 and its HEP value for 23 minutes. "T7-3 \#3 refers to Table 7-3, item 3. These are typical abbreviations used in HRA event trees.

Finally, the estimated total failure probability, $F_{T}$, of .1 is entered into the appropriate place in the system fault tree or system event tree per step 11 in Table $7-1$. 


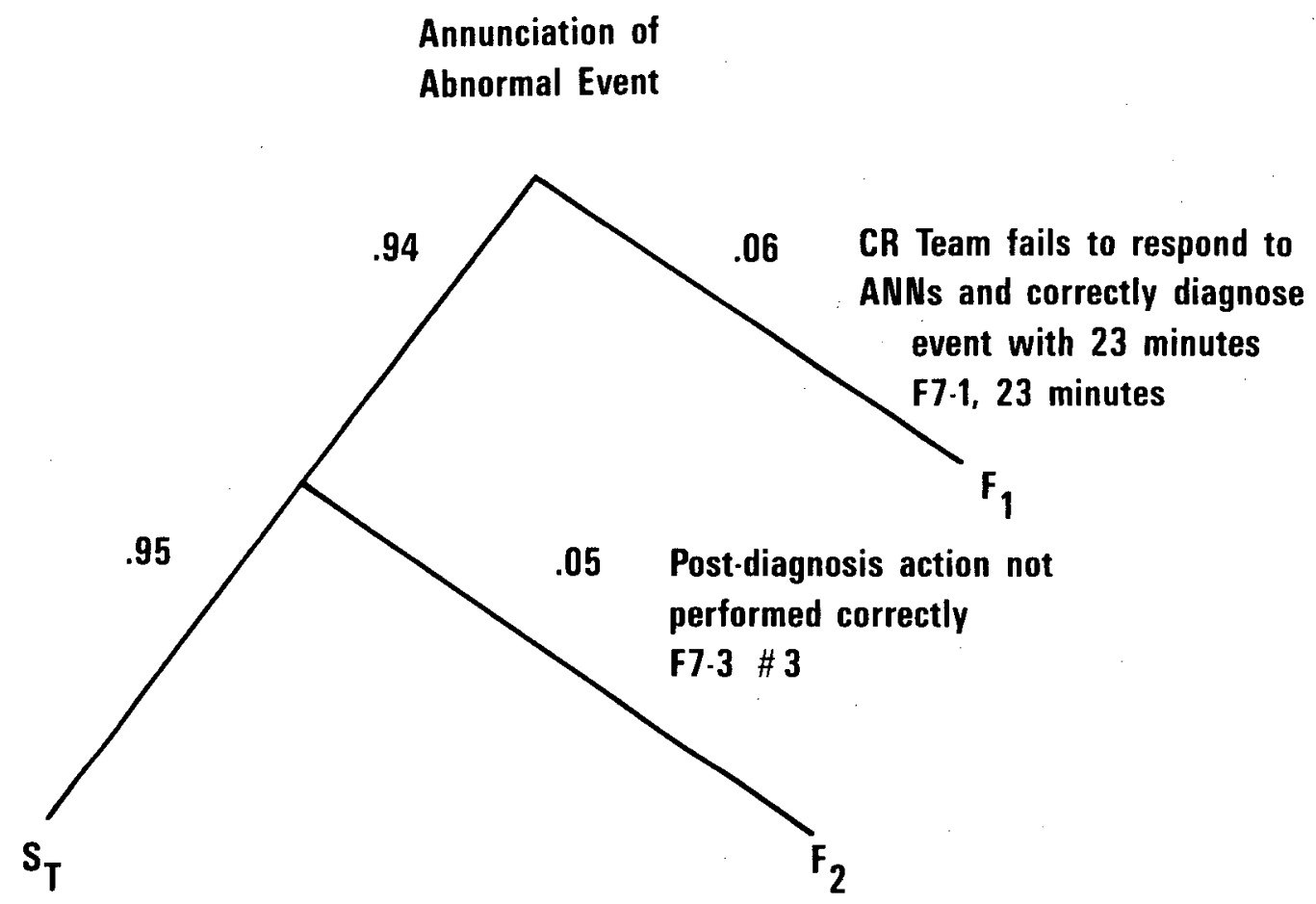

The screening $F_{T}$ is calculated as follows:

$$
\begin{aligned}
& \mathrm{S}_{\mathrm{T}}=.94 \times .95=.893 \sim .9 \\
& \mathrm{~F}_{\mathrm{T}}=1-\mathrm{S}_{\mathrm{T}} \sim .1
\end{aligned}
$$

$\underline{\text { or }}$

$$
\mathrm{F}_{\mathrm{T}}=\mathrm{F}_{1}+\mathrm{F}_{2}=.06+(.94 \times .05)=.107 \sim .1
$$

Following is a check on the correct logic and arithmetic:

$$
\begin{aligned}
& \mathrm{S}_{\mathrm{T}}+\mathrm{F}_{\mathrm{T}} \text { must equal } 1.0 \\
& .893+.107=1.0
\end{aligned}
$$

The above illustrates an advantage of not rounding until the final answer. Figure 7-2 HRA Event Tree for a Hypothetical Post-Accident Screening HRA 


\section{General Information}

The ASEP Nominal HRA procedure for post-accident tasks differs from the ASEP Screening HRA procedure by incorporating changes to the latter to reduce undue conservatism. First, the nominal diagnosis model from NUREG/CR-1278 (reproduced here as Table 8-2 and Figure 8-1) is used in place of the screening diagnosis model (as shown in Table 7-2 and Figure 7-1). Rules for adjusting the nominal diagnosis model upwards or downwards are provided, with special credit given to the use of symptom-oriented EOPs which help reduce the amount of interpretation, diagnosis, and decision-making, as defined in Table 6-1. More credit is given for well-practiced responses to memorized immediate responses to emergency operating conditions.

Second, credit is allowed for error recovery factors (RFs) outside the control room. Allowance is permitted for the RFs afforded by having several people in the control room subsequent to an abnormal event. However, in case there is no opportunity for performing the requisite task analysis to determine the RFs, some general rules are provided which are intended to ensure conservatism.

Third, although it is still assumed that there will be at least a moderately high level of stress during at least the first two hours following the annunciation of an abnormal event, the analysis permits the use of different post-diagnosis action HEPs for the interaction of type of task (i.e., step-bystep and dynamic) and level of stress (i.e., moderately high stress and extremely high stress).

Fourth, it is assumed that if there is a novice person (i.e., one who has less than six month' experience on the tasks in question), a more experienced person will perform the critical actions in coping with an abnormal event. For example, if a control room operator is a novice, he would quickly be replaced by a more experienced person.

Use Table 8-1 as the basic procedure for the ASEP Nominal HRA for post-accident tasks. This procedure is intended to offer the opportunity for the analyst to use plant-specific information. Yet in keeping with one purpose of ASEP, the ability to perform an "accelerated PRA," certain generic estimates are employed, with rules on how to modify them if sufficient plant-specific information can be obtained. The nominal values in the tables in this section of the document are intended to err on the conservative side, when errors in estimation are made. However, the nominal values presumably avoid undue conservatism. If sufficient plant information cannot be obtained, the analyst should incorporate other conservatisms. For example, if he is not allowed to interview a sufficient number of operating personnel to have confidence that certain plant rules are always (or nearly always) carried out, he should employ a certain amount of healthy skepticism, and use upper uncertainty bounds (or some other adjustment factor) in place of the nominal values from the data tables, with appropriate documentation of the rationale behind these adjustments. Sensitivity analyses should be performed to show the impact of 
such adjustments and to show the need for a more detailed HRA if these adjustments, or any estimates, have a major influence on the overall PRA.

The following tables and figures present the background material for the ASEP nominal HRA procedure for post-accident tasks. Table 2-1 defines skill-, rule-, and knowledge-based behavior. Table 6-1 and Figure 6-1 define and diagram the interrelationships of diagnosis and other cognition-related terms. Figure 6-3 shows the required time relationships among $\mathrm{T}_{0}, \mathrm{~T}_{\mathrm{m}}, \mathrm{T}_{\mathrm{a}}$, and $\mathrm{T}_{\mathrm{d}}$.

With two exceptions, error factors (EFs) for estimated HEPs are assigned per Table 20-20 from NUREG/CR-1278, or are included in the copies of tables from that document. The exceptions are the assignment of an EF of 5 rather than 10 for post-diagnosis dynamic tasks performed under moderately high stress (items 4 and 7 in Table 8-5). The rationale is one of simplification and to avoid low uncertainty bounds to ensure greater conservation.

\section{The Procedure}

The detailed procedure for the nominal HRA for post-accident tasks is presented in Tables 8-1, 8-2, 8-3, 8-4, and 8-5 and Figure 8-1. Table 8-1 provides the basic procedure, with references to other tables (or figures), as appropriate. Table 8-2 and Figure 8-1, the nominal diagnosis model, and Table 8-3, guidelines for adjusting the nominal diagnosis model, are taken from NUREG/CR-1278. Table 8-4 is the Annunciator Response Model; it is a copy of Table 20-23 from NUREG/CR-1278, as revised 9/1/85. A hypothetical example at the end of this chapter shows how the ASEP nominal HRA procedure might be used for post-accident tasks. 
Table 8-1 Procedure for Nominal HRA of Post-Accident Tasks ( $1 / 6$ )

1. Review the definitions and concepts in Table 2-1 (defining skill-, rule-, and knowledge-based behavior) and Table 6-1 and Figure 6-1 (defining and diagramming diagnosis and other cognition-related terms), and Figure 6-3 (showing the required time relationships of $\mathrm{T}_{0}, \mathrm{~T}_{\mathrm{m}}, \mathrm{T}_{\mathrm{a}}$, and $\mathrm{T}_{\mathrm{d}}$ ).

2. For the following cases, assess HEP $=1.0$ for the entire HRA for the abnormal event in question; no further HRA is required:

a. Critical skill-based or rule-based post-diagnosis actions are not described in written procedures. (Details of skill-based actions are not required to be written if they can be classified as "skill-ofthe-craft" - see the prefatory section "Definitions of Technical Terms.") This assessment is used even though it may be required for personnel to have memorized these actions. Instead, they would likely refer to the written procedures at a later time during the usual checking to see that all immediate emergency actions had been performed correctly. : Lack of written procedures is considered indicative of inadequate quality assurance, and is the justification for this assessment. However, it is not expected that the diagnosis aspect of every abnormal event would be treated in detail in the written procedures; the rules in this table for assessing diagnosis HEPs are treated separately.

b. The required instrumentation fails to support diagnosis or postdiagnosis behavior, or the instrumentation is inaccurate (i.e., misleading).

3. Using systems analysis methods, and referring to Figure 6-3, estimate $T_{m}$, the maximum allowable time to have correctly diagnosed an abnormal event and to have completed the necessary human actions following $\mathrm{T}_{0}$, the annunciation (or other compelling signal) of an abnormal event. For definitions and interactions of diagnosis and related terms, see Table 6-1 and Figure 6-1.

4. Identify the actions required to successfully cope with the abnormal event, once a correct diagnosis has been made.

5. For post-diagnosis actions to be performed in the control room area, attempt to measure travel time and manipulation time in the training simulator or by means of a timed walk-through in the plant control room. To the extent that such measurements are not possible, employ the following rules:

a. If there is a requirement to use written procedures, i.e., the human actions to be performed cannot be assumed to be committed to memory, assess a 5-minute delay, after correct diagnosis, before the first of the required post-diagnosis actions will be initiated. 
Table 8-1 Procedure for Nominal HRA of Post-Accident Tasks (p2/6)

b. Assess 1 minute as the required travel and manipulation time combined for each control room (CR) control action taken on the primary operating panels which are normally in visual access of the CR operator. An example is activation of the manual trip button.

c. For required control actions on other than the primary $C R$ operating panels, assess 2 minutes as the required travel and manipulation time for each such control action.

d. Consider the effects of planned assignments of personnel to monitor particular panels for specified abnormal events.

e. If estimates of time are obtained from operating personne1, double them.

6. For travel and manipulation times outside the control room, use simulated measures (e.g., walk-throughs) to estimate the time required to get to the appropriate location and to perform the necessary post-diagnosis actions. If estimates from operating personnel must be used, double them.

7. Sum the estimated times from steps 5 and 6 to calculate $T$, the time needed to get to a particular location plus the time needed ${ }^{a}$ to perform required actions once a diagnosis of an initiating event has been made. To avoid unreasonably large estimates of $T$, take into account planned or likely assignments of different actions to different people, which could result in some actions being carried out in the same time period.

8. Calculate $T_{d}=T_{m}-T_{a}$, which is the allowable time for a diagnosis which permits the performance of the required actions within the total allowable time, $T_{m}$. See Figure 6-3.

9. Using $T$, select the appropriate diagnosis HEP from Figure 8-1 or Table 8-2. This nominal diagnosis HEP is a joint HEP representing the performance of the entire control room crew. Adjust the HEP upwards or downwards, using the rules stated below. For such adjustments, employ new uncertainty bounds (UCBs) based on the UCBs listed for the same numbers in Table 8-2 or shown in Figure 8-1. Diagnosis HEPs assume that any novice operator (i.e., one with less than 6 months' experience in the tasks in question) would be replaced by a more experienced one.

a. This diagnosis HEP is considered the probability of misdiagnosis which will result in a core damage accident.

b. For the case of more than one abnormal event occurring closely in time (i.e., within 10 minutes), use Table 8-2 to estimate the diagnosis HEP for the second or subsequent simultaneously occurring 
Table 8-1 Procedure for Nominal HRA of Post-Accident Tasks (p3/6)

abnormal event. Use the guidelines below in judging whether to assess more than one abnormal event in an accident sequence being evaluated.

1) If the emergency operating procedure (EOP) appropriate to the accident sequence does not specifically describe any additional events being evaluated, including any change in the status of the event being evaluated, reapply the nominal diagnosis model. In deciding whether to reapply this model, it is not necessary that the EOP specifically name the additional abnormal events or changes in status; it must, however, lead the operators to cope successfully with these conditions if they correctly use the EOP. That is, the EOP must enable the operators to figure out what to do (or be led to the correct actions) in coping with the accident sequence details being evaluated. If the EOP does provide this guidance, do not assess the need for a second or subsequent diagnosis .

2) If the second or third abnormal event occurs "closely in time" with the first event, as defined in the first footnote to Table 8-2, employ the second or third column in the table. If the second or third abnormal event occurs later, and it can be judged that the control room personnel are no longer actively engaged in diagnosing and/or planning the responses to cope with the first event, use the first column for such additional events.

3) It is judged that the third column is sufficiently conservative to employ for any additional abnormal events assessed as occurring "closely in time," as defined in Table 8-2.

4) Note that in Table 8-2 $T_{0}$ refers to a compelling signal of the initiation of any abnormal event and that a probability of 1.0 is assumed for observing that there is some abnormal situation. This assumption must be evaluated for the second and subsequent abnormal events in an accident sequence. Use the Annunciator Response Model (Table 8-4) to estimate the probability that the signal of second and subsequent abnormal events will indeed be noticed.

c. Use the rules in Table 8-3 to adjust the diagnosis HEP upwards or downwards for the first, second, and third abnormal events in any accident sequence.

d. If symptom-oriented EOPs are available and if the criteria itemized below are met, adjust the diagnosis HEP downwards by using HEPs from the lower bound of the nominal diagnosis curve (Figure 8-1) as the new set of nominal HEPs.

1) The initiating event in question is covered in these EOPs. 
Table 8-1 Procedure for Nominal HRA of Post-Accident Tasks (p4/6)

2) The appropriate control room operators have been trained in the use of symptom-oriented EOPs.

3) Credit for symptom-oriented EOPs is to be given only for the percentage of operators estimated to actually use these EOPs rather than trust to their memory. If there is no other basis to use to estimate this percentage, assess a .5 probability that the appropriate operator will use the symptom-oriented EOPs in a step-by-step manner, rather than depend on his memory. For the fraction of operators assessed as depending on memory, give no credit for symptom-oriented EOPs. Use Table 8-3 to adjust the new values, as appropriate.

4) These EOPs are well designed (e.g., no gaps, inconsistencies, potentially misleading or confusing statements or paths, or requirements to follow more than one path simultaneously without prompts from one path to another).

e. For the diagnosis HEP for reactor vessel/containment critical parameters which operating personnel must commit to memory, use the lower bound values in Figure 8-1 or Table 8-2 only if the recognition of these parameters can be classified as skill-based behavior per Table 2-1; otherwise, use the nominal values. Use Table 8-3 to adjust the new values, as appropriate.

As an example of reactor vessel/containment critical parameters which all the CR reactor operators commit to memory, the four critical parameters at LaSalle Boiling Water Reactor (BWR) nuclear power plant (NPP) are:

- Check reactor power level. It must not exceed 1188.

- Check the water level in the core. It must not be below 12.5 inches above instrument zero.

- Check reactor pressure. It must not be over 1046 psi.

- Check containment temperature and pressure. Temperature must not be over 110 degrees and pressure must not be over 1.69 psi.

f. If it can be determined that all control room operators are trained to quickly initiate a manual scram signal with the SCRAM switches when the annunciation of an automatic scram has occurred, or when an immediate indication of a failure to scram has occurred, and given that there is a written procedure (see item 2.a), assess a negligible probability of a diagnosis error, and instead assess only the failure to perform the correct switching action given that a correct diagnosis has occurred. Assume that any correct activation of the SCRAM switches will occur within one minute of the annunciation of a call for an automatic scram. In the case of BWRs, the same argument applies to manual activation of the switch which precludes early closure of the Main Steam Isolation Valves (MSIVs) due to low steam pressure if the same signals and training for manual activation of 
Table 8-1 Procedure for Nominal HRA of Post-Accident Tasks ( $p 5 / 6)$

the MSIV preclude switch (often called MODE switch) can be assumed. However, in the latter case, assume complete dependence (CD) between the activation of the manual SCRAM switch and the MSIV preclude switch. The above assessment is equivalent to assigning an HEP of .001 (from Table 8-5, item 10) for failure to correctly perform the manual switching action (or, for BWRs, actions).

g. As noted earlier, the diagnosis HEPs in Table 8-2 (or Figure 8-1) are joint HEPs representing the performance of the entire control room crew. In some cases, especially during the first 30 minutes into an abnormal event, task analysis information may indicate that the diagnosis HEPs, even the lower uncertainty bounds, are unduly conservative. As noted on p 12-21 of NUREG/CR-1278, "...it must be determined whether the abnormal event being analyzed is one in which diagnosis errors are credible. It may be judged that for a particular abnormal event, the operating personnel are so well versed in recognizing the pattern of stimuli associated with the event that the cognitive aspect of behavior may be very small. The decision of the analyst should be based on the frequency with which each member of each control room operations team practices diagnosing the abnormal event in question." In addition, the advent of symptom-oriented EOPs may convert formerly knowledge-based behavior (e.g., diagnosis) into rule-based behavior. The analyst may judge that the diagnosis aspect of some particular event is negligible because of the combination of training and procedures. In making such a judgment, the analyst must understand that there is a risk of an overly optimistic assessment of human behavior, especially considering the likely stressful nature of abnormal events no one believes will ever occur. However, the analyst has the option of ignoring diagnosis error and using only post-diagnosis errors (Table 8-5). Such assessments should be fully documented.

10. Select the appropriate HEP(s) for post-diagnosis action(s) from Table 85. Items a, b, and c present some guidelines for assessing whether a set of post-diagnosis actions constitutes a dynamic or step-by-step task. Items $d, e$, and $f$ present some guidelines for assessing whether a set of post-diagnosis actions is to be assessed as being performed under moderately high stress or extremely high stress. The nominal stress level is not used in the ASEP HRA Procedure for post-accident tasks. Item $g$ refers to the use of the doubling rule for time-stress. In addition to the guidelines below, also see the prefatory section "Definitions of Technical Terms" for definitions of dynamic and step-by-step tasks and for moderately high and extremely high stress levels. For more explanation of levels of stress and their effects on performance, see Chapter 17 of NUREG/CR-1278.

a. If some safety-related system fails after the operating crew is using the EOP, reclassify as dynamic any step-by-step tasks related to the use of the EOP. 
Table 8-1 Procedure for Nominal HRA of Post-Accident Tasks (p6/6)

b. If the criteria in item 9.d above related to extra credit for the use of symptom-oriented EOPs cannot be met, assess any post-diagnosis actions related to these EOPs as dynamic.

c. If an individual operator must perform more than one task simultaneously without good cues for when he must shift from one task to another, assess each task as dynamic even though each task separately might be classified as step-by-step.

d. At least a moderately high level of stress is assessed for a minimum of 2 hours after the initiation of an abnormal event.

e. The occasion of a large loss-of-coolant accident is assessed as resulting in extremely high stress until such time as recirculation is established, at which time moderately high stress is assessed.

f. Extremely high stress is assessed for occasions in which more than two primary safety systems fail to function. However, if it can be determined that frequent simulator training has made control room personnel very familiar with the accident sequence being evaluated, the lower bound of the estimated HEP may be assessed.

g. If time stress is present, the doubling rule is assessed, i.e., when an operator is required to take some corrective action in moderately to extremely high stress conditions with very limited time available to take the corrective action, if the first action is ineffective, his HEP for each succeeding corrective action doubles (up to the limit of 1.0). The doubling rule applies to repeated attempts to perform the same task as well as to related tasks done by the same person.

11. Calculate the estimated total-failure probability, $F_{T}$, by adding the diagnosis HEP (Step 9) to the HEP(s) for carrying out the required postdiagnosis action(s) (Step 10). If this calculation results in a totalfailure probability greater than 1.0 , use 1.0 .

12. Enter the $F_{T} s$ in the appropriate system fault trees or system event trees, paying special attention that the dependence effects identified for human' actions are preserved in the way the $\mathrm{F}_{\mathrm{T}}$ s are used. See Chapter $5^{\prime}$ of NUREG/CR-1278 for guidelines. 


\section{SCREENING DIAGNOSIS MODEL}

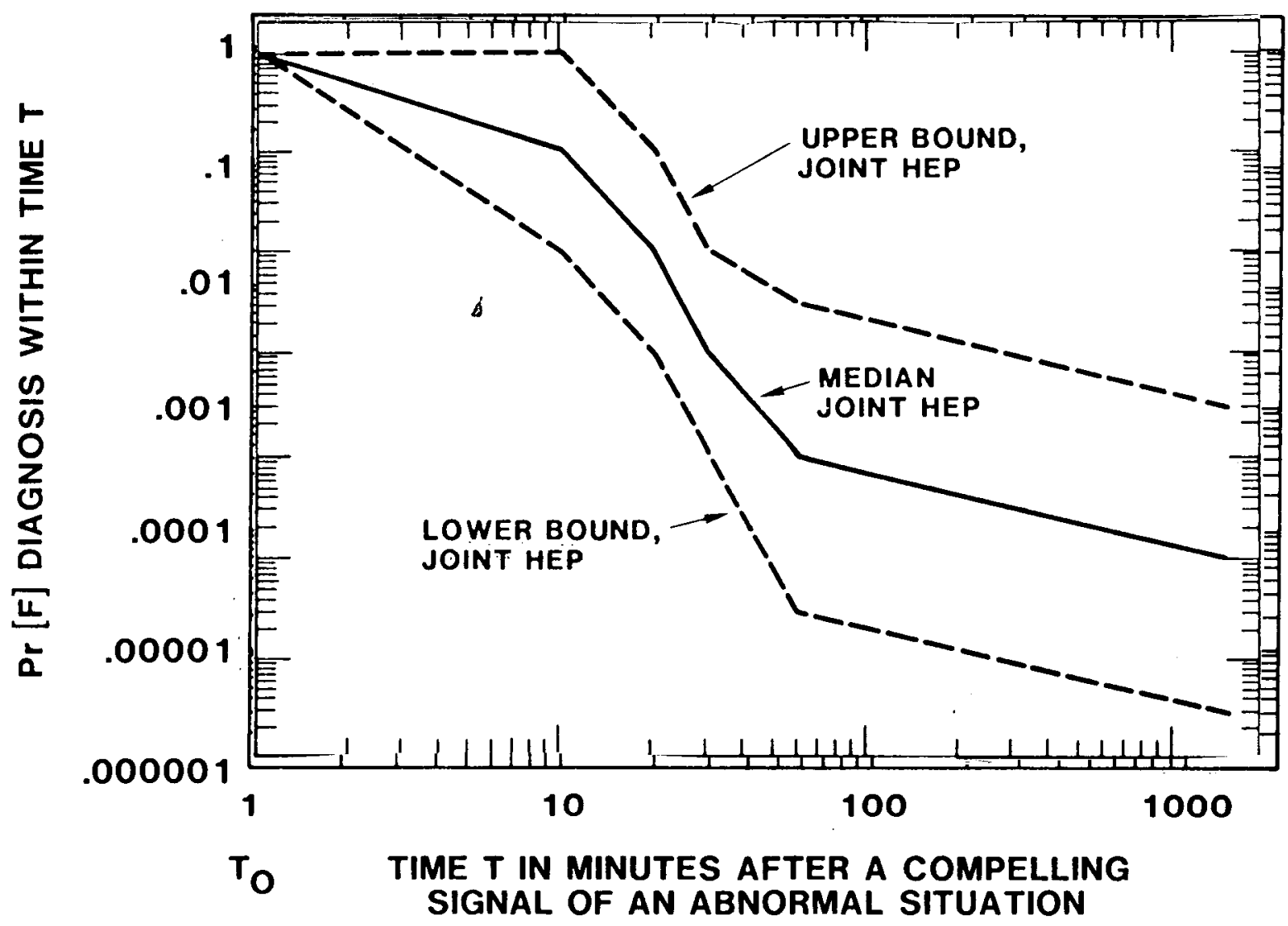

Figure 8-1 Nominal Model of Estimated HEPs and UCBs for Diagnosis Within Time $T$ of One Abnormal Event by Control Room Personnel

(Revised copy of Figure 12-4 from NUREG/CR-1278. The revision corrects the labeling of the ordinate in the figure so that the "1" occurs where the three lines in the figure meet at the ordinate, as shown in the above figure.) 
Table 8-2 Nominal Model of Estimated HEPs and EFs for Diagnosis Within Time T by Control Room Personnel of Abnormal Events Annunciated Closely in Time*

(Copy of Table 20-3 from NUREG/CR-1278 with appropriate changes to figure and table numbers)

\begin{tabular}{|c|c|c|c|c|c|c|c|c|c|c|c|c|c|}
\hline Item & $\begin{array}{c}I \\
\text { (Minutesst* } \\
\text { after } I_{0}^{+} \text {) }\end{array}$ & $\begin{array}{l}\text { Median joint } \\
\text { HEP }^{++} \text {for } \\
\text { diagnosis of a } \\
\text { single or the } \\
\text { first event }\end{array}$ & EF & $\begin{array}{l}1 \\
1 \\
1 \\
1 \\
1\end{array}$ & Item & $\begin{array}{c}\mathrm{T} \\
\text { (Minutes } \\
\text { after } \mathrm{T}_{0}^{+} \text {) }\end{array}$ & $\begin{array}{l}\text { Median joint } \\
\text { HEP }^{++} \text {for } \\
\text { diagnosis of } \\
\text { the second } \\
\text { event }\end{array}$ & $E F$ & $\begin{array}{l}1 \\
1 \\
1 \\
1 \\
1\end{array}$ & Item & $\begin{array}{c}\mathrm{T} \\
\text { Minutes } \\
\text { after } \mathrm{T}_{0}^{+}\end{array}$ & $\begin{array}{l}\text { Median joint } \\
\text { HEP for for } \\
\text { diagnosis of } \\
\text { the third } \\
\text { event }\end{array}$ & $E F$ \\
\hline (1) & 1 & 1.0 & -- & 1 & $(7)$ & 1 & 1.0 & -- & 1 & (14) & 1 & 1.0 & -- \\
\hline (2) & 10 & .1 & 10 & I & ( 8$)$ & 10 & 1.0 & -- & 1 & (25) & 10 & 1.0 & -- \\
\hline (3) & 20 & .01 & 10 & I & (9) & 20 & .1 & 10 & 1 & (16) & 20 & 1.0 & -- \\
\hline \multirow[t]{2}{*}{ (4) } & 30 & .001 & 10 & 1 & (10) & 30 & .01 & 10 & i & (17) & 30 & .1 & 10 \\
\hline & & & & I & (11) & 40 & .001 & 10 & 1 & (18) & 40 & .01 & 10 \\
\hline \multirow[t]{2}{*}{ (5) } & 60 & .0001 & 30 & 1 & (12) & 70 & .0001 & 30 & 1 & & & & \\
\hline & & & & i & & & & & 1 & (20) & 80 & .001 & 30 \\
\hline \multirow[t]{2}{*}{ (6) } & 1500 & .00001 & 30 & 1 & (13) & 1510 & .00001 & 30 & 1 & & & & \\
\hline & & & & 1 & & & & & 1 & (21) & 1520 & .00001 & 30 \\
\hline
\end{tabular}

* "Closely in time" refers to cases in which the annunciation of the second abnormal event occurs while the control room personnel are still actively engaged in diagnosing and/or planning the responses to cope with the first event. This is situation-specific, but for the initial analysis, use "within 10 minutes" as a working definition of "closely in time."

Note that this model pertains to the CR crew rather than to one individual.

The nominal model for diagnosis includes the activities listed in Table 6-1 as "perceive," "discriminate," "interpret," "diagnosis," and the first level of "decision-making." The modeling includes those aspects of behavior included in the Annunciator Response Model in Table 8-4; therefore, when the nominal model for diagnosis is used, the annunciator model should not be used for the initial diagnosis. The annunciator model may be used for estimating recovery factors for an incorrect diagnosis or for the signals for additional abnormal events.

* For points between the times shown, use the medians and EFs from Figure 8-1 for the first event, and interpolate between the tabled values for the second or third events.

${ }^{+} T_{0}$ is a compeliling signal of an abnormal situation and is usually taken as a pattern of annunciators. A probability of 1.0 is assumed for observing that there is some abnormal situation.

${ }^{++}$Table 8-3 presents some guidelines to use in adjusting or retaining the nominal HEPs presented above 
Table 8-3 Guidelines for Adjusting Nominal Diagnosis HEPs from Table 8-2

(Copy of Table 12-5 from NUREG/CR-1278)

Item General Rules

(1) Use upper bound if:

(a) the event is not covered in training,

or

(b) the event is covered but not practiced except in initial training of operators for becoming licensed,

or

(c) the talk-through and interviews show that not all the operators know the pattern of stimuli associated with the event.

(2) Use lower bound if:

(a) the event is a well-recognized classic (e.g., TMI-2 incident), and the operators have practiced the event in the simulator requalification exercises,

and

(b) the talk-through and interviews indicate that all the operators have a good verbal recognition of the relevant stimulus patterns and know what to do or which written procedures to follow.

(3) Use nominal HEP if:

(a) the only practice of the event is in simulator requalification exercises and all operators have had this experience,

or

(b) none of the rules for use of upper or lower bound apply. 
Table 8-4 The annunciator Response Model: Estimated HEPs* for Multiple Annunciators Alarming Closely in Time***

(Copy of Table 20-23 from NUREG/CR-1278, as revised September 1, 1985)

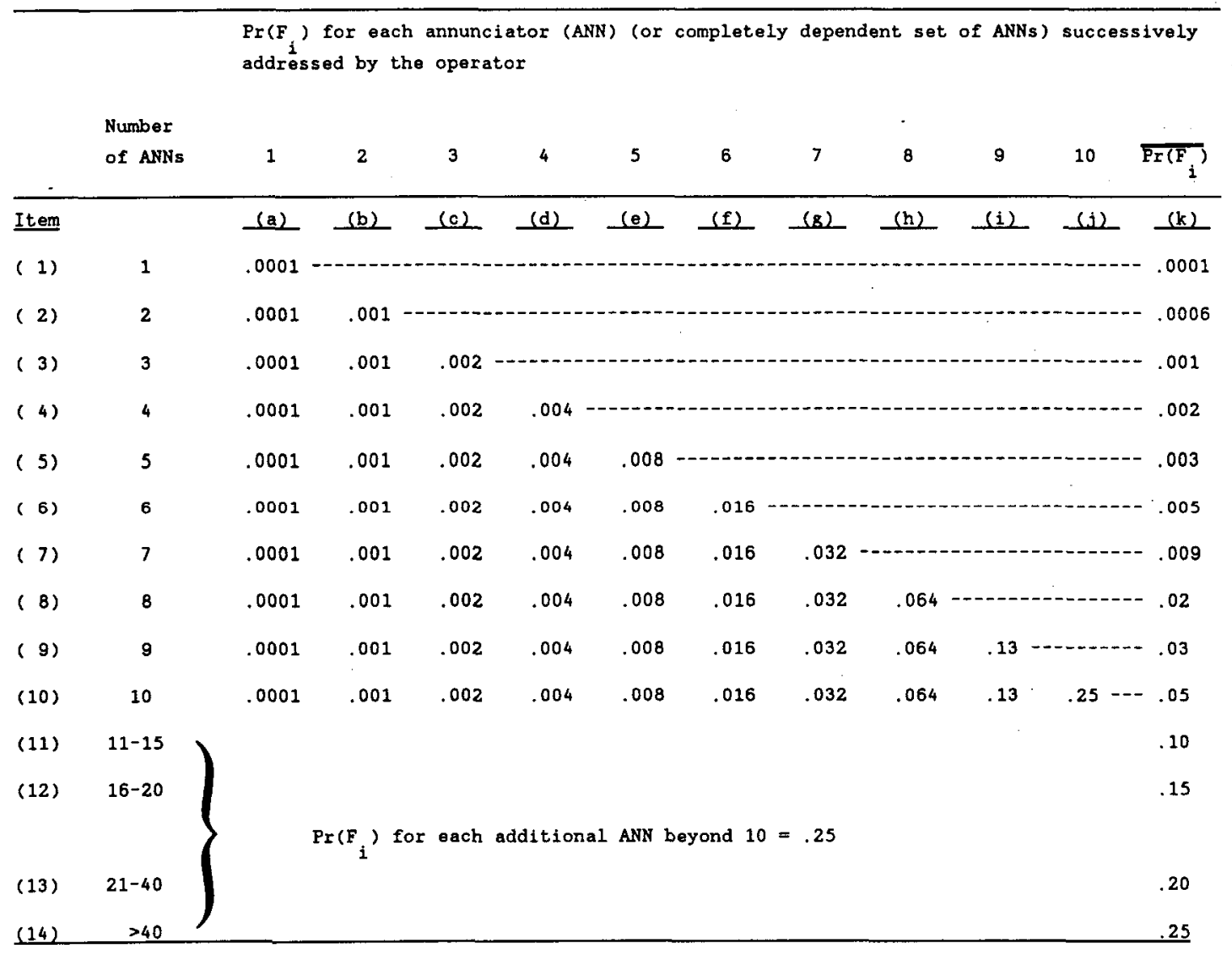

*The HEPs are for the fallure to initiate some $k$ ind of intended corrective action as required. The action carried out may be correct or incorrect and is analyzed using other tables. The HEPs include the effects of stress and should not be increased in consideration of stress effects.

EF of 10 is assigned to each $\operatorname{Pr}\left(F_{i}\right)$ or $\overline{\operatorname{Pr}\left(F_{i}\right)}$. Based on computer simulation, use of an $E F$ of 10 for $\overline{\operatorname{Pr}(F)}$ yields approximately correct upper bounds ${ }^{i}$ for the 95 th percentile. The corresponding lower bounds are too high; they are roughly equivalent to 20 th-percentile rather than the usual 5 th-percentile bounds. Thus, use of an $E F$ of 10 for the mean $\operatorname{Pr}\left(F_{i}\right)$ values provides a conservative estimate since the lower bounds are biased high.

"Closely in time" refers to cases in which two or more annunciators alarm within several seconds or within a time period such that the operator perceives them as a group of signals to which he must selectively respond.

+ $\overline{\operatorname{Pr}(F)}$ is the expected $\operatorname{Pr}(F)$ to initiate action in response to a randomly selected ANN (or completely dependent set of ANNs) in a group of ANNs competing for the operator's attention. It is the arithmetic mean of the $\operatorname{Pr}\left(F_{i}\right) s$ in a row, with an upper limit of .25. The $\overline{\operatorname{Pr}(F)}$ column assumes that all of the ANN (or completely dependent sets of ANNs) are equal in terms of the probability of being noticed. See page 11-52, paragraph 2 , in NUREG/CR-1278 if this assumption does not hold. 
Table 8-5 Assessment of Nominal HEPs for Post-Accident Post-Diagnosis Actions ( $1 / 2)$

$\underline{\text { Item }} \underline{\mathrm{HEP}} \underline{\mathrm{EF}}$ Action*

(1) 1.0 -- Perform a critical skill-based or rule-based action correctly when no written procedures are available. (Details of skillbased actions are not required to be written if they can be classified as "skill-of-the-craft" $* *$.) This assessment is used even though it may be required for personnel to have memorized these actions. Instead, they would likely refer to the written procedures at a later time during the usual checking to see that all immediate emergency actions had been performed correctly. (See Table 2-1 for definitions.)

(2) var. - - If sufficient information can be obtained per a task analysis, as described in Chapter 4 of NUREG/CR-1278, use the data tables in Chapter 20 of NUREG/CR-1278, adjusted for the effects of dependence, stress, and other performance shaping factors (PSFs), and error recovery factors (RFs) per the search scheme in Chapter 20. If this level of information cannot be obtained because of scheduling or other restrictions, use the remainder of this table.

Items (3), (4), and (5) present HEPs for the original performer of the action, and must be adjusted for the effects of other operators and recovery factors (items 6 -9). These HEPs are for failure to correctly perform a critical post-diagnosis procedural action as part of a "step-by-step task" $* *$ or a "dynamic task" $* *$ done under "moderately high stress" $* *$ or "extremely high stress" $x *$. See item 10 in Table 8-1 for guidelines on how to apply these terms. It is assumed that "novice personnel" would be replaced by "skilled personnel" for critical actions.

(3) .02 5 Perform a critical action as part of a step-by-step task done under moderately high stress.

(4) .055 Perform a critical action as part of a dynamic task done under moderately high stress or a step-by-step task done under extremely high stress.

(5) .255 Perform a critical action as part of a dynamic task done under extremely high stress.

*The HEPs are for independent actions or independent sets of actions in which the actions making up the set can be judged to be completely dependent. Other levels of dependence among actions can be assessed by the analyst, using one or more methods for assessing dependence described in Chapter 10 of NUREG/CR-1278.

$\times \times$ See the prefatory section "Definitions of Technical Terms" for definitions of these frequently misunderstood terms. 
Table 8-5 Assessment of Nominal HEPs for Post-Accident

Post-Diagnosis Actions ( 2 2/2)

\section{Item $\underline{H E P} \quad \underline{E}$ Action*}

If recovery of above errors made by the original performer is still possible at the point of error action, use following HEPs (6), (7), or (8) and related task and stress categories for a second person who checks the performance of the original performer.

(6) .25 Verify the correctness of a critical action as part of a stepby-step task under moderately high stress.

(7) $.5 \quad 5$ Verify the correctness of a critical action as part of a dynamic task done under moderately high stress or a step-bystep task done under extremely high stress.

(8) 55 Verify the correctness of a critical action as part of a dynamic task done under extremely high stress.*

(9) var. - - If there are error recovery factors (RFs) in addition to the use of human redundancy in items (6), (7), and (8), the influence of these RFs must be assessed separately. For annunciator RFs, use the Annunciator Response Model in Table $8-4$.

(10) .001 10 Perform a post-diagnosis immediate emergency action for the reactor vessel/containment critical parameters, when (a) it can be judged to have been committed to memory, (b) it can be classified as skill-based actions per Table 2-1, and (c) there is a backup written procedure. Assume no immediate RF from a second person for each such action.

*'Theoretically, if the HEP for item (7) is assessed as .5, the HEP for item (8) should be larger, e.g., .75. However, as .5 is already so large, any increase in the estimated HEP is judged to be unduly conservative. 


\section{A Hypothetical Example of a Nominal HRA}

The following hypothetical, simple example illustrates the use of part of the nominal procedure (Table 8-1), including the use of the method for estimating the appropriate time relationships between diagnosis and post-diagnosis actions (Figure 6-3), the nominal diagnosis model (Figure 8-1 and Table 8-2), rules for adjusting the diagnosis HEPs (Table 8-3), the annunciator response model (Table 8-4), and the nominal rules for post-accident, post-diagnosis actions (Table 8-5). This example problem is intended merely to illustrate the method. For more realistic problems making use of the ASEP HRA Procedure, see the ASEP PRAs in Volumes 3 - 6 of NUREG/CR-4550.

Assume that the human responses to a particular abnormal event are to be analyzed, using a nominal HRA. Per step 4 in Table 8-1, it has been determined that there are four critical post-diagnosis actions of interest, which require the use of a written procedure, and the available procedure is a symptom-oriented EOP. The systems analysts have assessed a major failure unless all four actions are correctly performed. Three of the actions take place at the main control room panels, and the fourth requires going to the diesel generator room and making one switching action. In the accident sequence being evaluated, it is assumed that the required instrumentation and related displays work properly.

Per step 3 in Table $8-1$, the systems analysts have estimated $T_{m}$ as 30 minutes. Reference to Figure 6-3 (as cited in step 3 of Table 8-1) shows that the control room crew must have completed all the appropriate actions in the time interval between the annunciation of the event $\left(\mathrm{T}_{0}\right)$ and $\mathrm{T}_{\mathrm{m}}$, in this case, within 30 minutes.

Per steps 5 and 6 of Table 8-1, and using plant personnel as subjects, the human reliability analyst has estimated the total time for the completion of the switching action in the diesel generator room to take 10 minutes. This time includes the following three times: (1) the time required for control room personnel to communicate with the ready room and instruct the person who would have to take the action, (2) that person's actual travel time (at a fast walk), and (3) the simulated time to make the switching action, once the operator is in the diesel room. The times for the other three post-diagnosis actions are much shorter and they are done by different people. Therefore, these times are of no consequence in the analysis. Per step 7 of the table, $\mathrm{T}_{\mathrm{a}}$ is 10 minutes. $\mathrm{T}_{\mathrm{d}}$ is calculated per step 8 as $30-10=20$ minutes.

Step 9 of the table refers the analyst to Figure 8-1 or Table 8-2 to obtain the estimated unadjusted diagnosis HEP for the calculated 20 minutes that are available for the diagnosis. Table 8-2, item 3, shows that the assessed unadjusted diagnosis HEP is .01. Possible adjustments are considered by referring to the other statements in step 9 of Table $8-1$.

Step $9 . \mathrm{b}$ is consulted, and it is determined by the systems analysts that for the accident sequence to be evaluated, only one diagnosis is required. Per step 9.c, the adjustment rules in Table 8-3 are consulted. It is established by interviews with plant personnel and training personnel that the abnormal event in question is not routinely practiced. Thus, it is appropriate to use 


\section{$8-16$}

the upper bound of the diagnosis HEP, i.e., .1. Per step 9.d a study of the symptom-oriented EOP shows that it is not well designed per 9.d.4 in that the person responsible for using the EOP to direct the post-accident actions has to follow three paths simultaneously without any written prompts from one path to another. Therefore, no downward adjustment of the diagnosis HEP is warranted. Steps 9.e, 9.f, and 9.g are determined not to be relevant to this particular problem. Therefore, the final assessed diagnosis HEP is .1.

Step 10 in the table refers the analyst to Table 8-5 to estimate the HEPs for the four critical post-diagnosis actions. However, item 10.b in Table 8-1 directs the analyst to assess all post-diagnosis actions as dynamic. Reference to items $10 . \mathrm{d}$ through $10 . \mathrm{g}$ results in an assessment of a moderately high level of stress for carrying out these four actions. Therefore, per item 4 in Table 8-5, an HEP of .05 is assessed for the original performer of each of these actions. For those control room actions, there is a second person RF. Therefore, item 7 in the table is used to assess an HEP of .5 for the RF for these three actions. Although there is no second person RF for the action to be performed in the diesel room, failure to perform this action will result in an annunciated alarm in the control room. Table 8-4 is consulted to obtain the estimated HEP for this RF. The systems analysts and control room personnel estimate that if this alarm sounds, there will be 9 other alarms competing for the attention of the control room personnel at about the same time. It is further determined that there are no special rules that would direct the attention of the personnel to the particular annunciator in question. Therefore, the appropriate assessment of the HEP for this RF is .05 per item $10 . \mathrm{k}$ and the last footnote of Table 8-4.

Figure 8-2 shows an HRA Event Tree as one method of analysis per step 11 in Table 8-1. (For a description of this type of event tree, see "HRA event tree" in the section on "Definitions of Technical Terms" in the prefatory pages.) In the figure, the sources for the estimated HEPs are listed by table and item number in the table, e.g., T8-2 \#3 UB. In this case, the UB refers to the use of the upper bound of .1 rather than the nominal value of .01. The dashed lines show error recovery paths which rejoin the complete success path, $\mathrm{S}_{1}$. Thus, there are 5 success paths through the tree (with $\mathrm{S}_{1}$ representing the end point of 4 of these paths).

Finally, the estimated total failure probability, $F_{T}$, of .2 is entered into the appropriate place in the system fault tree or system event tree per step 12 in Table 8-1. It is reiterated that this example is hypothetical and not realistic. An $F_{T}$ of .2 would be unusually high for an uncomplicated accident sequence. 


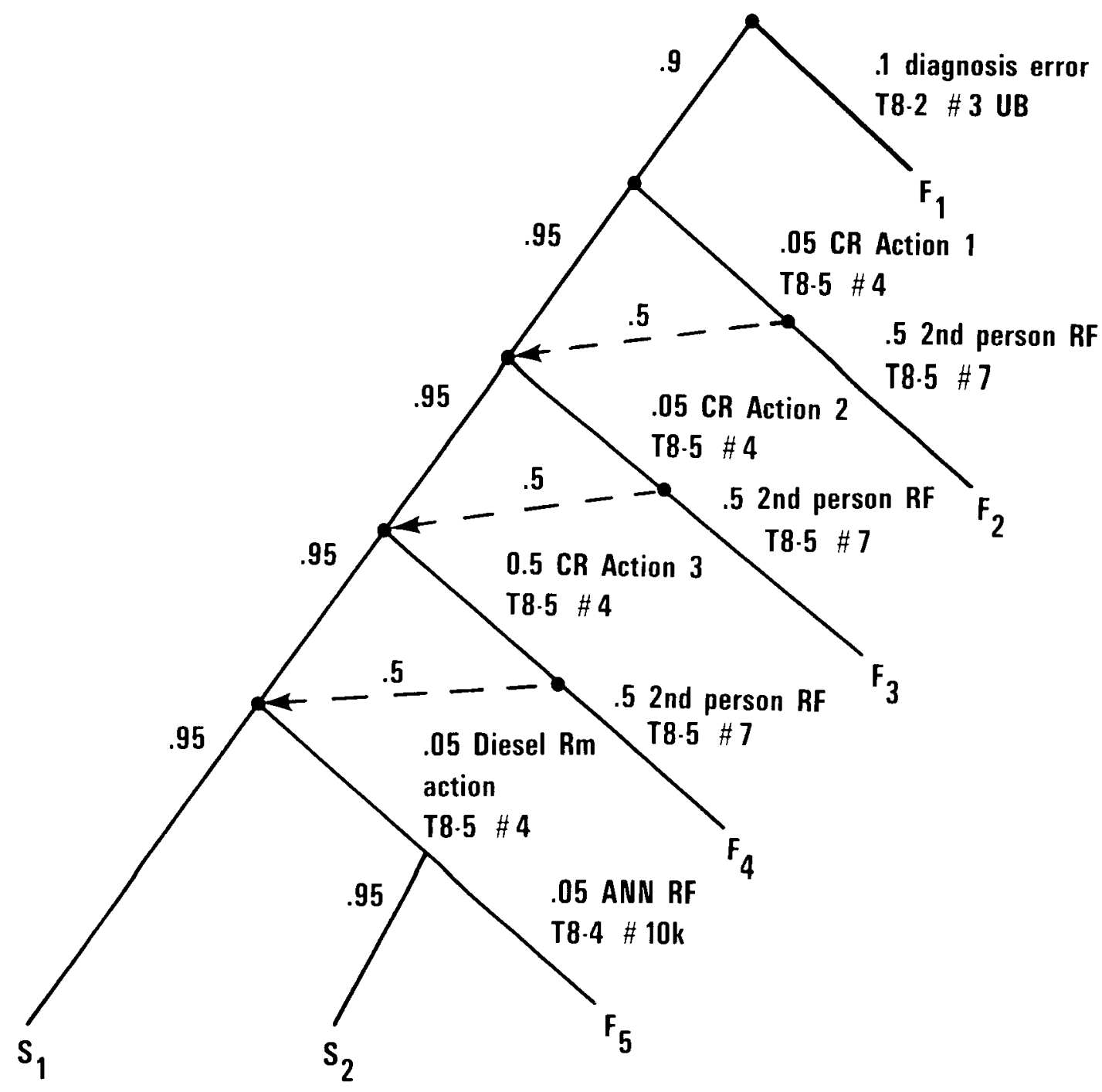

The nominal $\mathrm{F}_{\mathrm{T}}$ may be calculated as follows:

$$
\begin{aligned}
\mathrm{F}_{\mathrm{T}}= & \mathrm{F}_{1}+\mathrm{F}_{2}+\mathrm{F}_{3}+\mathrm{F}_{4}+\mathrm{F}_{5} \\
= & .1+(.9 \times .05 \times .5)+(.9 \times .95 \times .05 \times .5) \\
& +(.9 \times .95 \times .95 \times .05 \times .5)+(.9 \times .95 \times .95 \times .95 \times .05 \times .05) \\
= & .1661104 \sim .2
\end{aligned}
$$

or the approximate equation may be used:

$$
\mathrm{F}_{\mathrm{T}}-.1+3(.05 \times .5)+(.05 \times .05)-.1775-.2
$$

Figure 8-2 HRA Event Tree for a Hypothetical Post-Accident Nominal HRA 
PART IV. CONCLUDING COMMENTS

This part consists of Chapter 9, "Results of Tryouts of the ASEP HRA Procedure," and Chapter 10, "Conclusion." Chapter 9 describes experiences by four systems analysts and three human reliability analysts in using the new procedure in the four ASEP PRAs that were completed. Included are both positive comments and criticisms by these personnel. Chapter 10 presents a capsule evaluation of the procedure, with suggestions for additional study. 
CHAPTER 9. RESULTS OF TRYOUTS OF THE ASEP HRA PROCEDURE

\section{Overview}

The Accident Sequence Evaluation Program (ASEP) Human Reliability Analysis (HRA) Procedure was developed to meet a specific need - an HRA procedure sufficiently accurate for a "shortcut" probabilistic risk assessment (PRA) and usable by systems analysts with a minimum amount of guidance from human reliability analysts. Stated in another way, the developmental goal was to devise an HRA procedure requiring a minimum of judgment, and usable by PRA specialists with little or no background in human performance technology. This chapter presents some information on the results of several tryouts to test this developmental goal.

To state the bottom line first, the goal was realized in large part. Some changes to the original procedure used in the ASEP PRAs (Swain, 1985b and c) were made after the tryouts to better achieve the above goal of a rule-based procedure to be used by systems analysts. Nevertheless, the author and the other two human reliability analysts supporting the ASEP systems analysts believe it is still important that any HRA of a complex man-machine system be performed by an interacting team of specialists that includes a human reliability analyst with a strong background in human performance technology, e.g., a psychologist or human factors specialist who has been trained in HRA.

\section{The Tryouts}

The tryouts consisted of applications in both the ASEP and the Risk Methods Integration and Evaluation Program (RMIEP). The RMIEP application involved a systems analyst's use of the RMIEP HRA screening procedure for pre-accident tasks (Swain, 1985a) in the PRA of La Salle Unit 1, a boiling water reactor (BWR). This "screening procedure" (to be published as the section on "Screening Rules for the Human Reliability Analysis in the Risk Methods Integration and Evaluation Program" in Volume 5 of NUREG/CR-4832 (Payne et al, 1987 ) is nearly identical to the ASEP nominal HRA procedure for pre-accident tasks, as noted in Chapter 5, so it is a fair test also of the latter procedure. (See Appendix A for more information on the RMIEP "fine screening analysis" and its relationship to the ASEP HRA Procedure.)

The ASEP applications consisted of the use of the HRA nominal procedures for pre-accident and post-accident tasks in four different PRAs by four different systems analysts, with support from three human reliability analysts. The four plants were Surry Unit 1 and Sequoyah Unit 1, both pressurized water reactors (PWRs), and Peach Bottom Unit 2 and Grand Gulf Unit 1, both BWRs. The PRAs and the associated HRAs for these NPPs are reported in four different volumes (see NUREG/CR-4550 in References section). The ASEP systems analysts had no formal training in HRA, but one of them had some familiarity with THERP/Handbook, having worked with the author on other PRAs. The three human 
reliability analysts (including the author) were thoroughly versed in the THERP/Handbook method of HRA, as described in NUREG/CR-1278 and NUREG/CR-2254. Much of the detailed post-accident HRA was done by the systems analysts themselves. Most of the detailed pre-accident HRA was done by the human reliability analysts.

The tryouts, then, are of the ASEP nominal HRA procedure for both pre- and post-accident tasks. Neither ASEP screening HRA procedure has been tried out to date. After completion of the tryouts, the author talked with each of the other six analysts. The following two topics present the results of the tryouts of, respectively, the ASEP HRA procedure for pre-accident tasks and the ASEP HRA procedure for post-accident tasks.

\section{Tryouts of the ASEP HRA Procedure for Pre-Accident Tasks}

The La Salle PRA systems analyst and the author visited the plant and together studied the kinds of materials described in Table 5-1, "Procedure for Nominal HRA of Pre-Accident Tasks." In addition they interviewed persons in charge of maintenance, calibration, and restoration operations. Limited talk-throughs were obtained. The systems analyst stated that he had no problems in using the pre-accident HRA procedure, in this case the RMIEP screening procedure which is nearly identical to the ASEP nominal procedure. The author concurred in his use of the procedure and the results he obtained.

In the Surry and Sequoyah PRAs, a human reliability analyst (not the author) did most of the pre-accident HRA. The systems analysts reported that they did not do much pre-accident HRA because the human reliability analyst had classified the tagging procedure as a Level 1 procedure (from Table 20-15 in NUREG/CR-1278), and there were many annunciated signals for restoration errors, or post-maintenance tests that would also catch many of these kinds of errors. One of the systems analysts used the pre-accident procedure for only one task, one which did not have all the above recovery factors. He stated that in his opinion the assessed HEPs using the procedure were too optimistic. As his example, he noted that if two parallel systems were being calibrated, and if this involved the technician's going to different cabinets, there would be more than 2 minutes involved. Using Table 5-1, item \#9b, and Table 5-4, zero dependence was assessed. He would have preferred to use high dependence instead. (In the ASEP pre-accident HRA procedure, for greater conservatism, no levels of dependence between zero and high dependence are offered, unlike the positive dependence model in NUREG/CR-1278.)

The author agrees that in the above case high dependence would be a more conservative assessment, but in his opinion, the overall assessment is still conservative in view of the basic HEP of .03 and the conservative restrictions on credit given in the HRA for recovery factors. The procedure for the preaccident $H R A$ is intended to be a prescriptive, rule-based procedure, and with this purpose in mind, the author has attempted to keep the procedure as simple as possible. 
In the Grand Gulf PRA, a different human reliability analyst (not the author) did all of the pre-accident HRA. In the Peach Bottom PRA, the systems analyst did the pre-accident HRA. This analyst is the one who had prior familiarity with THERP/Handbook. He stated that the biggest problem was the time constraints in obtaining sufficient information to properly apply the tabled rules in the pre-accident nominal HRA procedure. In some cases, he was able to obtain sufficient information to get down to two cases (for example, in using Table 5-3) but not sufficient information to indicate which of the two cases was the appropriate one. So he picked an in-between value on a $\log$ scale, a conservative application. As stated also by two other analysts, this systems analyst noted that there were so many recovery factors that in most cases the impact of human errors was negligible in the pre-accident PRA. He concluded that the pre-accident HRA procedure was well-defined and well-understood.

The other two human reliability analysts experienced no problems in using the ASEP nominal HRA for pre-accident tasks, but this was to be expected because of their strong background in THERP/Handbook.

\section{Tryouts of the ASEP HRA Procedure for Post-Accident Tasks}

The systems analysts and the other two human reliability analysts experienced some minor problems in using this procedure. Most of these problems occurred because of inadequate or misleading definitions of terms in the earlier draft version (Swain, 1985c). The present version in this document has redefined some terms and provided some additional guidance in an attempt to respond to these users' problems.

One systems analyst stated that the choice of HEPs to use in Table 8-4 is too limited. Mostly he used the .02 HEP (item \#3 in the table) for the initial error, and a .2 HEP (item \#6 in the table) for the failure of the recovery factor. He felt there was not enough latitude for fine tuning. He did appreciate the .001 HEP for skill-based behavior (i.e., item \#10 in the table), but believes that there is too big a gap between this .001 and the .02 value above. The author acknowledges there is a big gap between the two HEPs, but believes that any attempt to provide detailed rules for "fine tuning" would defeat a primary purpose of the ASEP HRA Procedure, namely, to provide a short cut method. Instead, this is an area to which the skills of a qualified human reliability analyst could be applied.

All of the analysts had problems in deciding how often to apply the nominal diagnosis model (in Table 8-2) in any one accident sequence. Two analysts finally decided that for most sequences, the model should be applied only once, and that would be to get the operators to the correct EOP. Once into an EOP, the post-diagnosis actions (Table 8-4) would be appropriate. For example, one analyst noted that loss of steam generator cooling is one sequence of tasks spelled out in an EOP. However, some human actions he judged to be stand-alone actions, e.g., failure to switch service water screens to the other unit (the one not experiencing an abnormal event). He judged this to be an unusual case, as it seems to require something out of the ordinary. It is 
not called out as a step in the EOP the operators would be using. Therefore, he used the nominal diagnosis model for that particular necessity for the operators to decide what to do. In other words, in that particular accident sequence, he used the diagnosis model twice. This seemed reasonable to the author.

With the above comments in mind, Table 8-1 includes additional clarification on when to employ the nominal diagnosis model more than once in the same accident sequence. The clarification specifically is addressed to the judgment of when to use the second and third columns in Table 8-2. The clarifying statement is that if the emergency operating procedure (EOP) appropriate to the accident sequence does not specifically describe any additional abnormal events posited by the systems analyst, including any change in the status of the event being evaluated, then the nominal diagnosis model should be applied in all such cases. This does not imply that the EOP must specifically name the additional abnormal events or changes; it merely means that the EOP must lead the operators to cope successfully with these conditions if they correctly use the EOP. That is, the EOP must enable the operators to figure out what to do (or be led to the correct actions) in coping with the accident sequence details being evaluated by the systems analyst.

In addition, the new clarification specifically reminds the systems analyst to use Table 8-3 to adjust the nominal HEPs for the second or third abnormal events in an accident sequence. For example, assume that a second abnormal event in an accident sequence is being analyzed and it is judged that the nominal diagnosis model should be applied to that event. Further assume that the analyst determines that this particular event is not covered in training. Accordingly, per item \#1.a in Table 8-3, he should select the appropriate HEP of the second column from Table 8-2, and then assess its upper bound as the estimated nominal HEP. Thus, if the operating crew must diagnose this second abnormal event within 20 minutes, the HEP of .1 (item \#9) would be reassessed as 1.0 , the upper bound of .1.

After using the original post-accident procedure (Swain, 1985c), another systems analyst noted that the term "initiating event" was too restrictive when he used the nominal diagnosis model. By standard PRA usage, an initiating event is an abnormal event that requires a trip. Yet some abnormal events can occur subsequent to a trip, and would require that the second and third columns in Table 8-2 be used. So he suggested that the term "abnormal event" be substituted for the term "initiating event," and that the words "..or other subsequent system failures" be added to the definition of an abnormal event. (These changes have been made in the present document.)

This same analyst, as did others, experienced some confusion with the definitions of step-by-step tasks and dynamic tasks in NUREG/CR-1278. Accordingly, these definitions have been changed in the current document. He also suggested that if some system fails after an operator has selected the appropriate EOP and is using it, any step-by-step tasks related to the use of the EOP should be reclassified as dynamic. Other than the above difficulties, this systems analyst judged the post-accident HRA procedure to be reasonably straightforward. 
Except for the "big gap between .02 and .001 in Table 8-4," a11 of the above suggestions have been incorporated into the present document. In addition, some additional guidelines have been included in Table 8-1 to help the analyst make an assessment as to whether a post-diagnosis task is a step-by-step or a dynamic task. For example, if graphic aids such as symptom-oriented EOPs are poorly designed, the user's tasks should be judged to be dynamic. In one plant, it was noted that in one of the symptom-oriented graphic EOPs evaluated, the operator had to simultaneously follow three different paths with no cues as to when to switch from one to another path. The task therefore was assessed as a dynamic task. In another plant, for the HRA of Anticipated Transient Without Scram (ATWS) accident sequences, the author and another human reliability analyst decided to assess all post-diagnosis tasks as dynamic even for tasks which individually could be classified as step-by-step. Their rationale was the large number of interactions among tasks to be carried out by each operator in the control room, and the fact that different tasks had to be performed in the same time period by a given operator. The clarifications to Table $8-1$ are intended to reflect these kinds of assessments. In addition, this table has been modified to include an assessment of symptom-oriented EOPs as a new method for adjusting the nominal diagnosis model in Table 8-2.

One of the systems analysts considered an assessment of a .125 joint HEP (i.e., the .25 HEP from item \#5 in Table 8-4 multiplied by the .5 HEP from item \#8) to be unduly conservative. This analyst wanted to use the concept of negative dependence, but such usage would quickly result in unbelievably low joint HEPs (as discussed in Chapter 10 of NUREG/CR-1278). The analyst also believed that the author's HRA did not give sufficient credit for the operators' training to keep the core covered. In the context of the conservative nature of the ASEP PRA, the author disagreed with the systems analyst. The author told the systems analyst that because it is impossible to predict a11 possible modes of extraneous behavior in an accident sequence, he prefers to err on the conservative side in those assessments of behavior that can be identified as possible operator actions.

This systems analyst stated that apart from problems with definitions, and some differences in interpretation of tabled HEPs, the HRA procedure was very easy to use. Changes made in the current document are intended to reduce these kinds of problems to a manageable level, and also to respond to the comments of the other two human reliability analysts who wanted some revisions to some of the definitions.

\section{A Comparison of Estimated HEPs Using ASEP HRA Procedure and THERP/Handbook}

In the Grand Gulf PRA, the author and another human reliability analyst performed two different HRAs for the ATWS accident sequences evaluated in the systems analysis. One HRA was performed using the draft ASEP nominal HRA procedure for post-accident tasks (Swain, 1985c), and the other was performed using the procedure in Chapter 20 (and Chapter 12) in NUREG/CR-1278. The HEPs used in the PRA were taken from the first HRA using the ASEP procedure (see Appendix D, "ATWS Human Reliability Analysis," in Volume 6 of NUREG/CR-4550), 
as it resulted in somewhat more conservative estimates. This, of course, was a stated intention in developing the ASEP procedure.

Table 9-1 provides a sample of estimated HEPs for various ATWS human actions showing the differences between the two sets of estimates. Quite a bit of explanatory material that is in Appendix D of the Grand Gulf ASEP PRA is left out, as the purpose of the table is merely to show some differences in estimated HEPs. The first two examples show estimates for detailed actions leading to total failure estimated HEPs. These are followed by some selected total failure HEPs. (The titles of the total failure HEPs are underlined in the table.)

As illustrated by the second detailed example in the table ("HEP 3: Operator Fails to Initiate Standby Liquid Control (SLC)"), in some cases the ASEP HRA Procedure does not permit as detailed an analysis as is possible using THERP/Handbook. This is to be expected in view of the purpose of the former method for HRA. The estimated total failure HEPs listed in the table show that in three cases the estimates were identical for both procedures, but that in the four cases in which differences occurred, the ASEP HRA Procedure was more conservative, as it was designed to be. 
Table 9-1 A Comparison of Some Individual and Total Failure HEPs Using the ASEP HRA Procedure and THERP/Handbook ( $1 / 2) *$

ASEP THERP Description of Human Action or Total Failure HEP

HEP HEP

HEP 2: Operator Attempts Recirculation Pump Trip (RPT)

5E-2 1E-3 Control Operator (CO) fails to note that SRV red lights are on, which indicates that the MSIVs have closed.

5E-2 1E-3 CO fails to note that the turbine has tripped and he fails to instruct the Assistant $\mathrm{CO}$ ( $\mathrm{ACO}$ ) to RPT.

5E-2 1E-3 ACO fails to note that the turbine has tripped and this indication fails to cue him to RPT.

$1 E-4 \quad 0 \quad$ Total Failure of all thŗee above possibilities for gȩtting to an RPT [ASEP $\mathrm{F}_{\mathrm{T}}-(5 \mathrm{E}-2)^{3}$; THERP/Handbook $\mathrm{F}_{\mathrm{T}}-(1 \mathrm{E}-3)^{3}$ ].

HEP 3: Operator Fails to Initiate Standby Liquid Control (SLC)

5E-2 1E-3 ACO fails to note that power $>58$, as displayed on several analog meters and recorders next to the control rods display.

5E-2 1E-3 CO fails to remember he should ask ACO for a power reading, given that an ATWS has occurred and that the Main Steam Isolation Valves have closed, or, he fails to note step 3.3 in EP10 which addresses this issue.

5E-2 5E-2 Shift supervisor (SS) fails to compensate for the above two persons' failures when he arrives in the control room the presumed 5 minutes into the event.

$\sim 0 \quad$ Once the CO realizes he must initiate SLC, he fails to go to the SS's desk and retrieve the specially marked keys on the key ring and he fails to insert and turn one of the keys in the proper place in panel $\mathrm{P} 601$.

$1 \mathrm{E}-4 \quad-0$

Total Failure of failing to attempt to initiate an SLC system within 3 to 7 minytes after the initiating event [ASEP $\mathrm{F}_{\mathrm{T}}-(5 \mathrm{E}-2)^{3} ;$ THERP/Handbook $\left.\mathrm{F}_{\mathrm{T}}-(1 \mathrm{E}-3)^{2}(5 \mathrm{E}-2)\right]$.

* The titles of the total failure HEPs are underlined in the table. 


$$
9-8
$$

Table 9-1 A Comparison of Some Individual and Total Failure HEPs Using the ASEP HRA Procedure and THERP/Handbook ( $22 / 2) *$

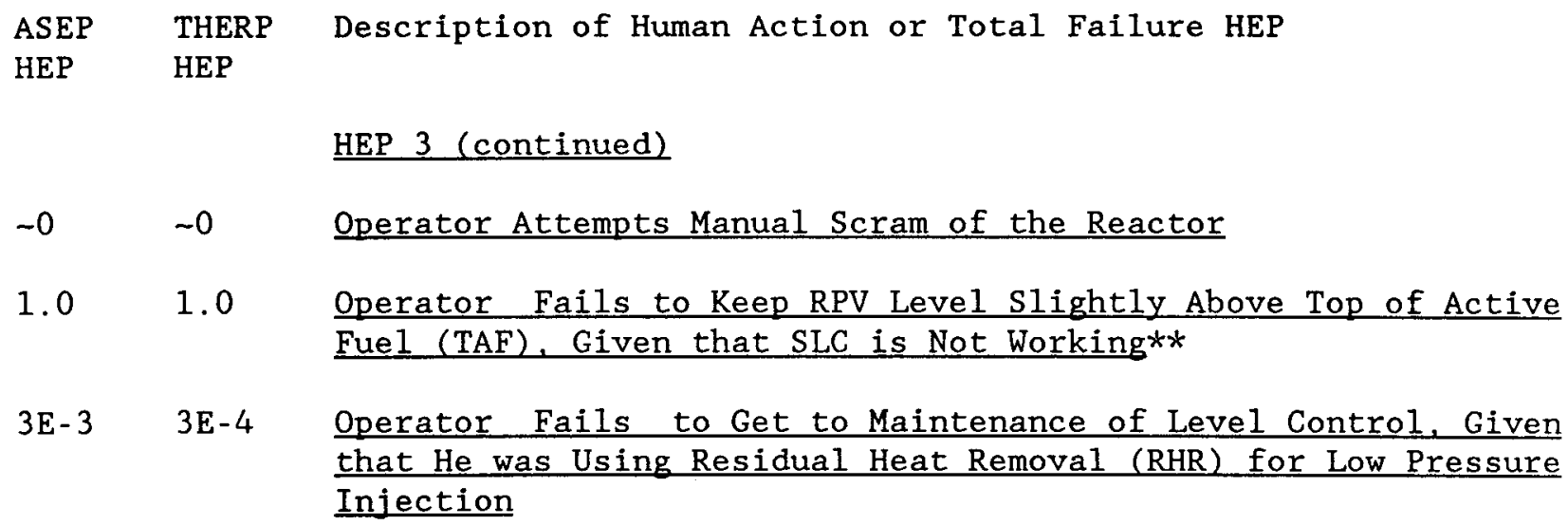

3E-3 3E-4 Operator Fails to Get to Maintenance of Level Control. Given that He was Using Residual Heat Removal (RHR) for Low Pressure Injection

2E-3 2E-4 Operator Fails to Initiate SPC, Given that He was Using RHR for Low Pressure Injection

1.01 .0 Operator Fails to Vent Containment, Given that SLC is Not Functioning and He Failed to Keep Reactor Pressure Vessel Level Slightly Above TAF

* The titles of the total failure HEPs are underlined in the table.

** The HEP of 1.0 was assessed because the training led to this inappropriate action. The training has since been revised. 
The goal in developing an ASEP HRA Procedure was to devise essentially a rulebased procedure (defined in Table 2-1) for human reliability analysis (HRA) that could be used by systems analysts to perform most of the analyses required for the HRA parts of a probabilistic risk assessment (PRA) of nuclear power plants. It was recognized that an abbreviated HRA procedure would of necessity leave out some aspects of performance shaping factors, the central raw material in applying a full-scale HRA using the THERP/Handbook approach described in NUREG/CR-1278. Thus, with an abbreviated procedure, especially a rule-based procedure, the best possible HRA cannot be achieved. Because PRAs are risk oriented, the author decided that the only possible approach would be one that would not likely underestimate risk. To develop a rule-based procedure, it was therefore necessary that develop rules that were conservative relative to the THERP/Handbook approach. On the other hand, he did not want to develop a procedure that would grossly overestimate the human contribution to system failures.

So, what is the result? The tryouts indicate that much, probably even most, of the ASEP HRA Procedure is indeed rule-based. Systems analysts who have had no formal training in human factors or psychology were able to apply the rules correctly in most cases. As might be expected, the rules for the pre-accident HRA are the most straightforward. They more closely approach the rule-based goal. Also, as might be expected, the rules for the post-accident HRA do require more judgment and soul searching to use. There is opportunity for an untutored systems analyst to overestimate the effectiveness of training for coping with the unusual, and to underestimate the effects of the stress of a very serious, unexpected abnormal event. For these reasons alone, the author strongly believes that any PRA which purports to consider the impact of human errors must include a specialist in HRA in the PRA team. This admonition is seconded by all of the systems analysts and o.ther reviewers of the ASEP HRA Procedure.

The tryouts also indicate that, as planned, the HRA results using the new procedure are somewhat more conservative than an HRA based on the more analytical and detailed THERP/Handbook approach. In some cases, differences between the two HRA procedures of as much as an order of magnitude in estimated human error probabilities (HEPs) occurred in the comparison made by the author and another human reliability analyst in the post-accident nominal HRA for one of the ASEP plants. However, in cases in which stress levels could be assessed as extremely high (e.g., when the hypothetical accident sequence included cases in which depended upon safety systems fail to work), there were no material differences in results between the ASEP HRA Procedure and THERP/Handbook. This outcome should not be surprising, as the considerable amount of uncertainty in predicting human behavior under these circumstances forces an analyst to be more pessimistic about human success.

An important plus for the ASEP HRA Procedure is that much of it can be done in a relatively. short period of time, with less manpower, than is the case using a full-scale HRA procedure. In a sense, then, the new procedure gains time and cost benefits by increasing the conservatism (pessimism) of the HRA. 
Another plus for the ASEP HRA Procedure is that, unlike NUREG/CR-1278, it does have a detailed fine-screening procedure for both pre-accident and post-accident HRAs. These screening procedures come the closest to completely rulebased procedures. But even though the ASEP HRA Procedure does include a screening procedure, the author prefers to think of the nominal ASEP HRA procedures as constituting another set of fine-screening tools for quickly identifying main sources of human error contribution in a PRA. Those human error events assessed as having a material impact in the systems analysis can then be subjected to a full-scale analysis. It may be determined that some simulator data are required to fill in some gaps in the post-accident HRA, as was done in the LaSalle PRA (Weston et al, 1987). A more detailed evaluation of the training and practice levels of plant personnel in coping with specific abnormal events may be justified on the basis of the analysis using the ASEP HRA Procedure. Or a more detailed HRA using THERP/Handbook may provide the necessary additional detail, especially to suggest changes in training, written procedures, work operations, or equipment to materially reduce estimated human error probabilities for original errors and recovery factors.

The ASEP HRA Procedure also provides some guidance for post-accident HRA not found in NUREG/CR-1278, namely, in assessing the effects of the relatively new symptom-oriented emergency operating procedures and of memorized immediate emergency actions, and in clarifying the use of the nominal diagnosis model. Therefore, for a full-scale HRA using the THERP/Handbook approach, parts of the ASEP HRA Procedure should also be used.

Despite a reduction in the amount of expert judgment required for the new procedure, both it and the THERP/Handbook procedure on which it is based have limitations in the knowledge-based behavior domain, notably in the methodology for a post-accident HRA. Both procedures rely on speculative time-reliability curves that are only marginally related to real data (see the discussion in Chapter 12 of NUREG/CR-1278). The author regards this approach as a temporary expedient until considerably more data from simulators can be obtained and properly calibrated to real world events. For the post-accident situation, data from training simulators offer the only viable possibility for collecting sufficient data for statistical treatment. Examples of data from nuclear power plant training simulators useful for PRA are presented in Beare et al, 1984, and in Weston et al, 1987. In these studies, funded by the U.S. Nuclear Regulatory Commission, human error rates based on error relative frequencies were collected. The first study shows HEPs for post-diagnosis actions that agree well with estimated HEPs in Chapter 20 of NUREG/CR-1278. The latter study includes diagnosis HEPs as a function of time from a compelling signal. For several simulated abnormal events, the data revealed a log-normal relationship that approximates that in the nominal diagnosis model (Figure 8-1 and Table 8-2). Although these data must be carefully evaluated when extrapolating them to operations in real plants, such data represent "the only game in town" for collecting statistically meaningful data on post-accident human behavior. Until regulatory agencies and utilities are willing to allocate considerably more time and funds to obtain sufficient data on human performance, human reliability analysis will continue to have a large amount of expert judgment behind the estimated effects of human errors in nuclear power plants. While this type of reliance is certainly not unique to HRA in the PRA.field, the author believes it is to be regretted. 
To sum up, the ASEP HRA Procedure is workable, it requires less manpower and time than THERP/Handbook, it fills in some gaps in NUREG/CR-1278, it is somewhat more conservative than the THERP/Handbook approach to HRA, and it meets a need for an abbreviated HRA procedure that is sufficiently accurate for most purposes of PRA. For those who prefer to use the more complete THERP/Handbook approach, the following treatment in the ASEP HRA Procedure should be incorporated into that approach: the new set of definitions of technical terms, the pre-accident and post-accident screening procedures, the uncertainty bounds propagation computer program, and the treatment of multiple abnormal events, immediate emergency actions, and symptom-oriented procedures. 
REFERENCES

Beare, A. N., R. E. Dorris, C. R. Bovell, D. S. Crowe, and E. J. Kozinsky, A Simulator-Based Study of Human Errors in Nuclear Power Plant Control Room Tasks, General Physics Corporation and Sandia National Laboratories, NUREG/CR-3309, US Nuclear Regulatory Commission, Washington, DC, January 1984 .

Bell, B. J., and A. D. Swain, A Procedure for Conducting a Human Reliability Analysis for Nuclear Power Plants, Sandia National Laboratories, NUREG/CR2254, US Nuclear Regulatory Commission, Washington, DC, May 1983.

Bertucio, R. C., M. D. Quilici, J. Young, and F. T. Harper, Analysis of Core Damage Frequency from Internal Events: Surry, Unit 1, Sandia National Laboratories, NUREG/CR-4550/Volume 3, US Nuclear Regulatory Commission, Washington, DC, January 1987.

Bertucio, R. C., J. Held, T. Lehy, and F. T. Harper, Analysis of Core Damage Frequency from Internal Events: Sequoyah. Unit 1, Sandia National Laboratories, NUREG/CR-4550/Volume 5, US Nuclear Regulatory Commission, Washington, DC, 1987 in press.

Brune, R. L. and M. Weinstein, Procedures Evaluation Checklist for Maintenance, Test, and Calibration Procedures, Human Performance Technologies, Inc., and Sandia National Laboratories, NUREG/CR-1369, Revision 1, U.S. Nuclear Regulatory Commission, Washington, DC, September 1982 .

Brune, R. L. and M. Weinstein, Checklist for Evaluating Emergency Procedures Used in Nuclear Power Plants, Human Performance Technologies, Inc., and Sandia National Laboratories, NUREG/CR-2005, Revision 1, U.S. Nuclear Regulatory Commission, Washington, DC, March 1983.

Drouin, M. T., J. L. La Chance, B. J. Shapiro, F. T. Harper, and T. A. Wheeler, Analysis of Core Damage Frequency from Internal Events: Grand Gulf. Unit 1, Sandia National Laboratories, NUREG/CR-4550/Volume 6, US Nuclear Regulatory Commission, Washington, DC, 1987 in press.

Flemming, K. N., A. Mosleh, D. L. Acey, B. O. Y. Lyde11, M. B. Sattison, J. Staub, D. W. Stillwell, and D. J. Wakefield, Classification and Analysis of Reactor Experience Involving Dependent Events, Pickard, Lowe and Garrick, Inc., EPRI NP-3967, Electric Power Research Institute, Palo Alto, CA, June 1985

Goodstein, L. P., "Discriminative Display Support for Process Operations," pp 433-449, in J. Rasmussen and W. B. Rouse (eds), Human Detection and Diagnosis of System Failures, New York: Plenum Press, 1981.

Hannaman, G. W. and A. J. Spurgin, Systematic Human Action Reliability Procedure, NUS, Inc., EPRI NP-3583, Electric Power Research Institute, Palo Alto, CA, June 1984. 
Harper, F. T., M. T. Drouin, and A. L. Camp, Analysis of Core Damage Frequency from Internal Events: Methodology Guidelines, Sandia National Laboratories, NUREG/CR-4550/Volume 1, US Nuclear Regulatory Commission, Washington, DC, 1987 in press.

Harper, F. T., M. T. Drouin, and A. L. Camp, Analysis of Core Damage Frequency from Internal Events: Summary Report, Sandia National Laboratories, NUREG/CR-4550/Volume 2, US Nuclear Regulatory Commission, Washington, DC, 1987 in press.

Kolaczkowski, A. M., J. A. Lambright, W. L. Ferrell, N. G. Cathey, B. Najafi, and F. T. Harper, Analysis of Core Damage Frequency from Interna1 Events: Peach Bottom, Unit 2, Sandia National Laboratories, NUREG/CR-4550/Volume 4, US Nuclear Regulatory Commission, Washington, DC, October 1986.

Kolb, G. J., D. M. Kunsman, B. J. Bell, N. L. Brisbin, D. D. Carlson, S. W. Robertson, R. O. Wooton, S. H. McAhren, W. L. Ferrell, W. J. Galyean, and J. A. Murphy, Interim Reliability Evaluation Program: Analysis of the Arkansas Nuclear one - Unit 1 Nuclear Power Plant, Vols. 1 and 2, Sandia National Laboratories, NUREG/CR-2787, US Nuclear Regulatory Commission, Washington, DC, June 1982.

MIL-STD-1472C, Military Standard, Human Engineering Design Criteria for Military Systems, Equipment and Facilities, U.S. Dept. of Defense, Washington, DC, 10 May 1984 (through Notice 2).

NUREG-75/014, WASH-1400, Reactor Safety Study - An Assessment of Accident Risks in U.S. Commercial Nuclear Power Plants, U.S. Nuclear Regulatory Commission, Washington, DC, October 1975: Main Report; Appendix II - Fault Trees; and Appendix III. Failure Data.

NUREG-0700, Guidelines for Control Room Reviews, U.S. Nuclear Regulatory Commission, Washington, DC, September 1981.

NUREG/CR-1278 - see Swain and Guttmann, 1983

NUREG/CR-2254 - see Be11 and Swain, 1983

NUREG/CR-2300, PRA Procedures Guide, Vols, 1 and 2, American Nuclear Society, Institute for Electrical and Electronic Engineers, U.S. Nuclear Regulatory Commission, Washington, DC, January 1983.

NUREG/CR-4550, Analysis of Core Damage Frequency from Internal Events:

Volume 1, Methodology Guidelines, see Harper et al, 1987a.

Volume 2, Summary Report, see Harper et al, 1987b

Volume 3, Surry. Unit 1, see Bertucio et al, 1987a.

Volume 4, Peach Bottom. Unit 2, see Kolaczkowski et al, 1986.

Volume 5, Sequoyah Unit 1, see Bertucio et al, 1987b.

Volume 6, Grand Gulf, Unit 1, see Drouin et a1, 1987.

NUREG/CR-4832 - see Payne et al, 1987. 
Payne, A. C., Jr., [\& other authors as yet undetermined], Risk Methods Integration and Evaluation Program (RMIEP). Volume 5: [no title as yet for Vol. 5, which will contain data analysis, uncertainty analysis, quantification, and human reliability analysisl, Sandia National Laboratories, NUREG/CR-4832, U.S. Nuclear Regulatory Commission, Washington, DC, 1987 in preparation.

Rasmussen, J., "What can be Learned from Human Error Reports?" Ch. 7 in K. D. Duncan, M. M. Gruneberg, and D. Wallis (eds), Changes in Working Life, New York: Wiley \& Sons, 1980.

Rasmussen, J., "Models of Mental Strategies in Process Plant Diagnosis," in J. Rasmussen and W. B. Rouse (eds), Human Detection and Diagnosis of System Failures, New York: Plenum Press, 1981.

Rasmussen, J., Human Errors. A Taxonomy for Describing Human Malfunction in Industrial Installations, Ris $\phi-M-2304$, Ris $\phi$ National Laboratory, Roskilde, Denmark, August 1981.

Rasmussen, J., "Human Reliability in Risk Analysis," pp 143-170 in A. E. Green (ed), High Risk Safety Technology, New York: Wiley \& Sons, 1982.

Rasmussen, J. and M. Lind, "A Model of Human Decision Making in Complex Systems and Its Use for Design of System Control Strategies," pp 270-276 in Proceedings of the American Control Conference, June 1982.

Swain, A. D., Fifth Review Draft, Appendix H - Screening Rules for the Human Reliability Analysis of the Risk Methods Integration and Evaluation Program, Sandia National Laboratories, Albuquerque, NM, June 20, 1985. [Note: This draft procedure was used in the La Salle PRA, and will been published as a section in Volume 5 of NUREG/CR-4832 - see Payne et al, 1987.]

Swain, A. D., Draft 2 ASEP HRA Procedure for Pre-Accident Tasks, Sandia National Laboratories, Albuquerque, NM, August 23, 1985.

Swain, A. D., Draft 3 ASEP HRA Procedure for Post-Accident Tasks, Sandia National Laboratories, Albuquerque, NM, September 20, 1985.

Swain, A. D. and H. E. Guttmann, Handbook of Human Reliability Analysis With Emphasis on Nuclear Power Plant Applications, Sandia National Laboratories, NUREG/CR-1278, U.S. Nuclear Regulatory Commission, Washington, DC, August 1983.

Swain, A. D. and L. M. Weston, "An Approach to the Diagnosis and Misdiagnosis of Abnormal Conditions in Post-Accident Sequences in Complex Man-Machine Systems," in L. P. Goodstein, H. B. Andersen, and S. E. Olsen (eds), Mental Models. Tasks and Errors, London: Taylor \& Francis, 1987 in press.

WASH-1400, Reactor Safety Study - An Assessment of Accident Risks in U.S. Commercial Nuclear Power Plants, NUREG-75/014, U.S. Nuclear Regulatory Commission, Washington, DC, October 1975. 


\section{$11-4$}

Webster's New Collegiate Dictionary, Springfield, MA: G. \& C. Merriam Company, 1975.

Weston, L. M., D. W. Whitehead, and N. L. Graves, Risk Methods Integration and Evaluation Program (RMIEP) Methods Development: A Data-Based Methodology for Including Recovery Actions in PRA, Sandia National Laboratories, NUREG/CR-4834, U.S. Nuclear Regulatory Commission, Washington, DC, 1987 in press.

Whitehead, D. W., L. M. Weston, and N. L. Graves, "A Simulator-Based Model for Estimating Operator Diagnosis Failure Probabilities," Proceedings of the ANS Winter Meeting. Washington, DC, November 1986, American Nuclear Society, La Grange Park, IL, 1986 (in press). 
A - 1

APPENDIX A. RELATIONSHIP OF ASEP HRA PROCEDURE TO RMIEP SCREENING RULES

In early 1984 Sandia National Laboratories was tasked by the U.S. Nuclear Regulatory Commission to develop and/or evaluate probabilistic risk, analysis (PRA) methods for assessing risk from nuclear power plants. This effort, the Risk Methods Integration and Evaluation Program (RMIEP), includes methods for human reliability analysis (HRA). As part of this work, a procedure for a screening HRA was developed for RMIEP (Swain, 1985a). This new procedure was developed because there was no formal HRA screening procedure in any existing HRA procedure documentation.

The RMIEP HRA screening procedure consists of "screening rules" for pre-accident and post-accident tasks. These screening rules constitute a "fine screening analysis," as defined in the "Definitions of Technical Terms" in the prefatory pages. A fine screening analysis incorporates results of some level of task analysis at the plant in question. Obviously, the degree of fineness or coarseness of a screening procedure is relative. The RMIEP HRA screening procedure for pre-accident tasks represents a "very fine" screening tool, whereas the RMIEP HRA screening procedure for post-accident tasks is relatively more coarse.

Because screening analysis procedures are inherently conservative and require less analysis than conventional (i.e., nominal) analysis procedures, it was decided to use the RMIEP screening rules as the basis for developing a new HRA procedure for use in the ASEP PRAs. Because of its emphasis on plant-specific task analysis, ASEP systems analysts decided that the RMIEP screening rules for pre-accident tasks could be used, with only minor modification, as the nominal HRA procedure for pre-accident tasks. The modification consists of (1) allowing some credit (i.e., an error recovery factor - RF) for supervisory sign-off on a test and maintenance job, and (2) being more explicit about allowing greater credit for shiftly or daily checks should the standard credit result in a task which has a material impact in the systems analysis.

The RMIEP screening rules for post-accident tasks were judged to be too coarse (i.e., not sufficiently plant-specific) for use as the nominal HRA procedure for ASEP post-accident tasks. Accordingly, for post-accident tasks a new nominal HRA procedure was developed for ASEP, while the screening HRA procedure for ASEP is very similar to the RMIEP screening procedure, with some exceptions to increase the conservatism of the ASEP procedure.

To document the development of the ASEP HRA Procedure, Table A-1 shows the correspondence between RMIEP and ASEP tables and figures. The content of the two sets is not identical, but a study of the two sets will show a high degree of correspondence. The RMIEP table and figure numbers are from Swain (1985a). For additional documentation of the development of the ASEP HRA Procedure, especially the rationale for the BHEP of .03 and the treatment of RFs in the pre-accident procedure, see Swain (1985a) or the section on "Screening Rules for the Human Reliability Analysis in the Risk Methods Integration and Evaluation Program" in Volume 5 of NUREG/CR-4832. 


\section{A-2}

Table A-1 Correlation of Table and Figure Numbers Between ASEP HRA Procedure and RMIEP HRA Screening Rules*

ASEP RMIEP $\quad \underline{\text { Content }}$

\section{Pre-Accident HRA}

T5-1 T1 Steps to follow in applying the pre-accident procedure

T5-2 T2 Basic and optimum conditions for recovery factors in preaccident tasks, exclusive of within-person dependence effects

T5-3 T3 Applications of ASEP T5-2 or RMIEP T2, exclusive of withinperson dependence effects

T5-4 T4 Guidelines for assessing within-person dependence levels for pre-accident tasks

T5-5 T5 $\mathrm{F}_{\mathrm{T}} \mathrm{s}$ for ASEP T5-3 or RMIEP T3 BHEPs, modified for multiplecomponent systems, assuming dependence levels determined by using guidelines in ASEP T5-4 or RMIEP T4, and including RFs

$\underline{\text { Post-Accident HRA }}$

T2-1 T8 Definitions of skil1-, rule-, and knowledge-based behavior

T6-1 T7 Definitions of cognition-related terms and usage in the Handbook of Human Reliability Analysis (NUREG/CR-1278)

F6-3 F1 Time relationships between annunciation of an abnormal event, a correct diagnosis, and performing the required post-diagnosis within time $T$ of one abnormal event by control room personnel

T7-1 T6 Steps to following in applying the post-accident procedure

F7-1 F2 Initial-screening model of estimated HEPs and UCBs for diagnosis within time $T$ of one abnormal event by control room personnel

T7-2 T9 Initial screening mode1 of estimated HEPs and EFs for diagnosis within time $T$ by control room personnel of abnormal events annunciated closely in time

T7-3 T10 Assessment of screening HEPs for post-accident post-diagnosis actions

*RMIEP table (T) and figure (F) numbers are from Swain (1985a). 
APPENDIX B

COMPUTER PROGRAM FOR PROPAGATING UNCERTAINTY BOUNDS

THROUGH AN HRA EVENT TREE

\title{
Elizabeth Lee Frost \\ Sandia National Laboratories
}

\begin{abstract}
Program UCBs-Propagation (short title UCBs) performs the calculations described in Appendix A of NUREG/CR-1278 for propagating uncertainty bounds (UCBS) through an HRA event tree. The user should reference this report for a complete understanding of the routine.
\end{abstract}

UCBs, written for the VAX computer in Fortran 77, performs all calculations in double precision. Input and output utilize external data files.

Input: For input, UCBs accesses an external data file created by the user. The default file type is "DAT".

Al1 input data, except the title line, are list-directed or "freeformat". This simply means that data values are not restricted to specific columns; however, there must be at least one space between data values. Character variables, such as, DEPENDENCE LEVEL, must be enclosed in single quotes. The only acceptable entries for DEPENDENCE LEVEL are ' $Z D^{\prime}$ ', ' $L D$ ', 'MD', ' $H D$ ', and ' $C D$ ' for zero, low, moderate, high, and complete dependence.

For each event tree the following input data are required:

line 1 : Title - up to 78 characters

line 2 : NEFT (Number of end-failure-terms)

line 3 : LIMB array - containing number of limbs in each end-failure term (EFT). The number of entries on line 3 must equal NEFT.

line 4 : Data for EFT 1, 1 imb 1 , i.e.:

DEPENDENCE LEVEL

MEDIAN HEP

RATIO OF UPPER/LOWER BOUND

(refer to Table B-2)

line $n$ : Data for the last limb of the last EFT

Any number of data sets can appear in a single data file. 


\section{B - 2}

Output: UCBs creates a file for the results that has the same name as the input file with the file type "LIS".

When UCBs is executed it will prompt the user for:

Data file name (omit file type)

Additional data file (if any)

UCBs is simply a tool to perform the calculations to determine upper UCBs (UBs) and, lower UCBs (LBs) as described in Appendix A of NUREG/CR-1278. The data set appearing there is used here as an example. The HRA event tree is shown in Figure B-1 and its companion Table B-1 explains the failure limbs.

The data file that was used for the example in this appendix is given in Table B-2 with annotations to aid the user.

The output file obtained by executing UCBs appears in Table B-3 followed by the source listing in Table B-4. 


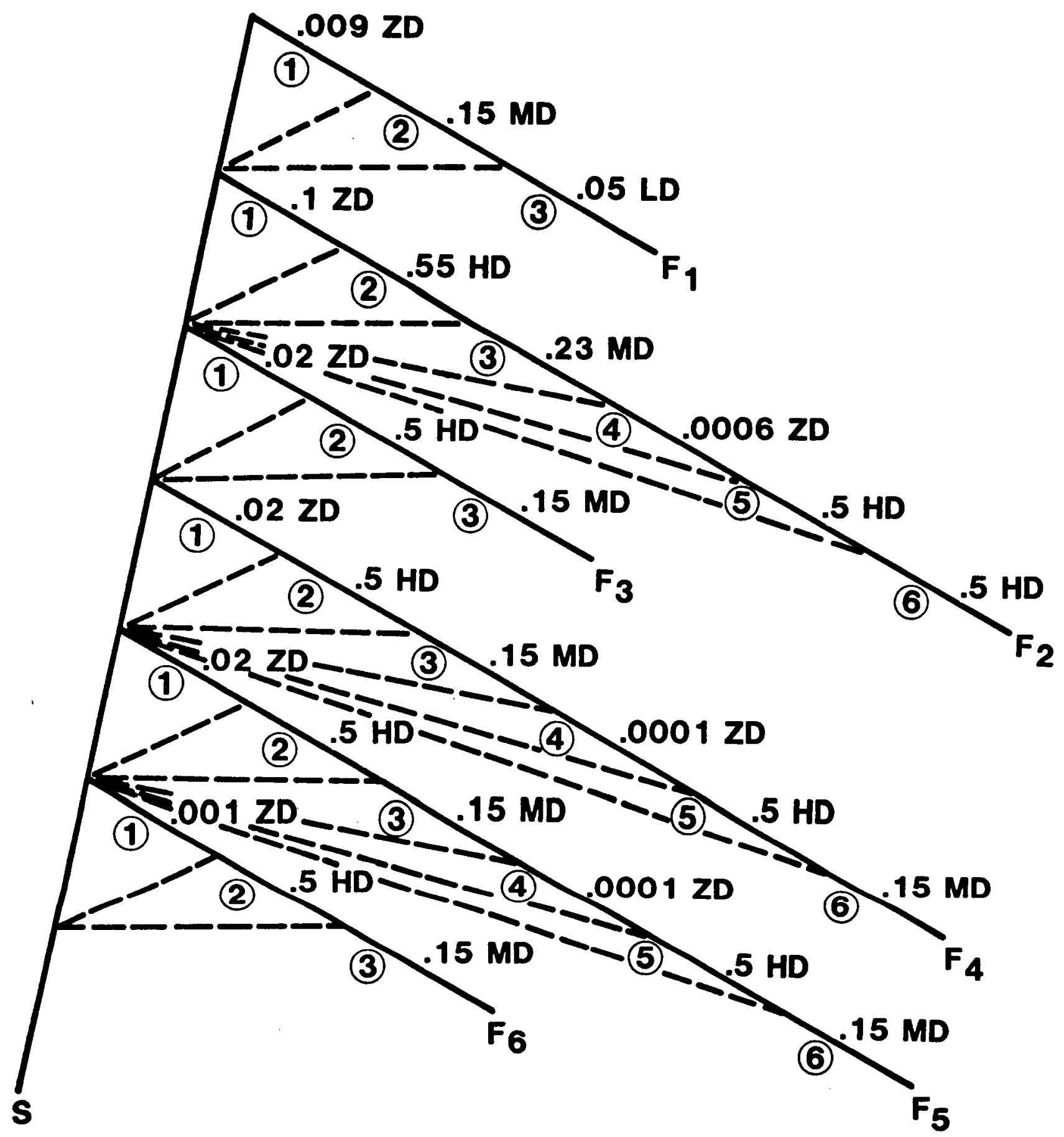

LEVEL OF DEPENDENCE IS SHOWN AFTER EACH HEP

Figure B-1 Expanded.HRA Event Tree for Loss of Steam Generator Feed (Copy of Figure A-2 from NUREG/CR-1278) 
Table B-1. Explanation of Failure Limbs in Figure B-1

(Copy of Table A-2 from NUREG/CR-1278)

Failure

$\operatorname{Limb}\left(F_{i j}\right)$

$F_{1,1}$

$F_{1,2}$

$\mathrm{F}_{1,3}$

$F_{2,1}$

$F_{2,2}$

$\mathrm{F}_{2,3}$

$\mathrm{F}_{2,4}$

$\mathrm{F}_{2,5}$

$\mathrm{F}_{2,6}$

$\mathrm{F}_{3,1}$

$\mathrm{F}_{3,2}$

$\mathrm{F}_{3,3}$

$\mathrm{F}_{4,1}$

$\mathrm{F}_{4,2}$

$\mathrm{F}_{4,3}$

$\mathrm{F}_{4,4}$

$\mathrm{F}_{4,5}$

$\mathrm{F}_{4,6}$

$F_{5,1}$

$\mathrm{F}_{5,2}$

$F_{5,3}$

$\mathrm{F}_{5,4}$

$\mathrm{F}_{5,5}$

$F_{5,6}$

$\mathrm{F}_{6,1}$

$F_{6,2}$

$F_{6,3}$

\section{Description}

R01 (reactor operator \#1) fails to note the relevant ANNs (annunciators) indicating the need to establish feed and bleed. R02 (reactor operator \#2) fails to correct RO1's error. Shift supervisor (SS) fails to correct RO1's error.

RO1 noted the relevant ANNs but makes an incorrect diagnosis and does not initiate feed and bleed.

RO2 fails to correct RO1's error.

SS fails to correct R01's error.

RO1 fails to note recovery ANNs.

R02 fails to correct R01's error.

SS fails to correct RO1's error.

RO1 omits step 2.4 in the procedure (long list, no checkoff)

RO2 fails to correct RO1's error.

SS fails to correct ROl's error.

RO1 omits step 2.5 in the procedure (long list, no checkoff)

RO2 fails to correct RO1's error.

SS fails to correct Rol's error.

RO1 fails to note loss of feed ANN.

RO2 fails to correct RO1's error.

SS fails to correct RO1's error.

R01 omits step 2.6 in the procedure (long list, no checkoff)

RO2 fails to correct RO1's error.

SS fails to correct R01's error.

R01 fails to note loss of feed ANN.

R02 fails to correct R01's error.

SS fails to correct ROI's error.

R01 fails to initiate high-pressure injection (HPI).

RO2 fails to correct RO1's error.

SS fails to correct R01's error. 
Table B-2 Sample Data File for Program UCBs-Propagation

\section{Data File}

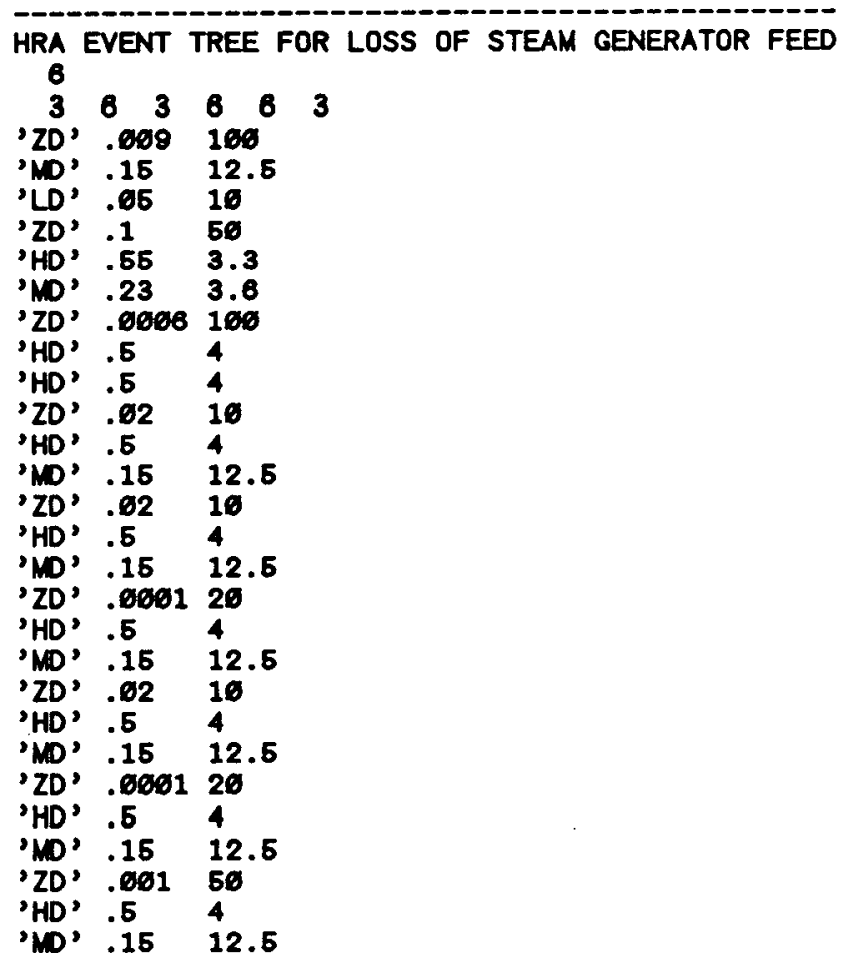

Explenetion

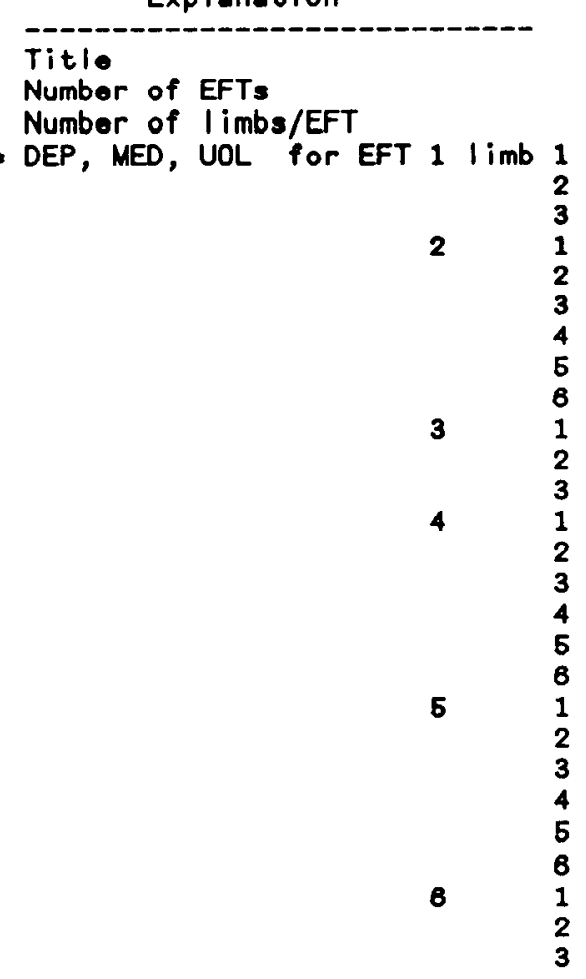

- NOTE: The data for the limbs must eppeer in the order in which they appear on the HRA ovent tree.

DEP = DEPENDENCE LEVEL

MED = MEDIAN HEP

UOL = RATIO OF UPPER OVER LOWER BOUND 
Table B-3 Program Output for Program UCBs-Propagation HRA EVENT TREE FOR LOSS OF STEAM GENERATOR FEED

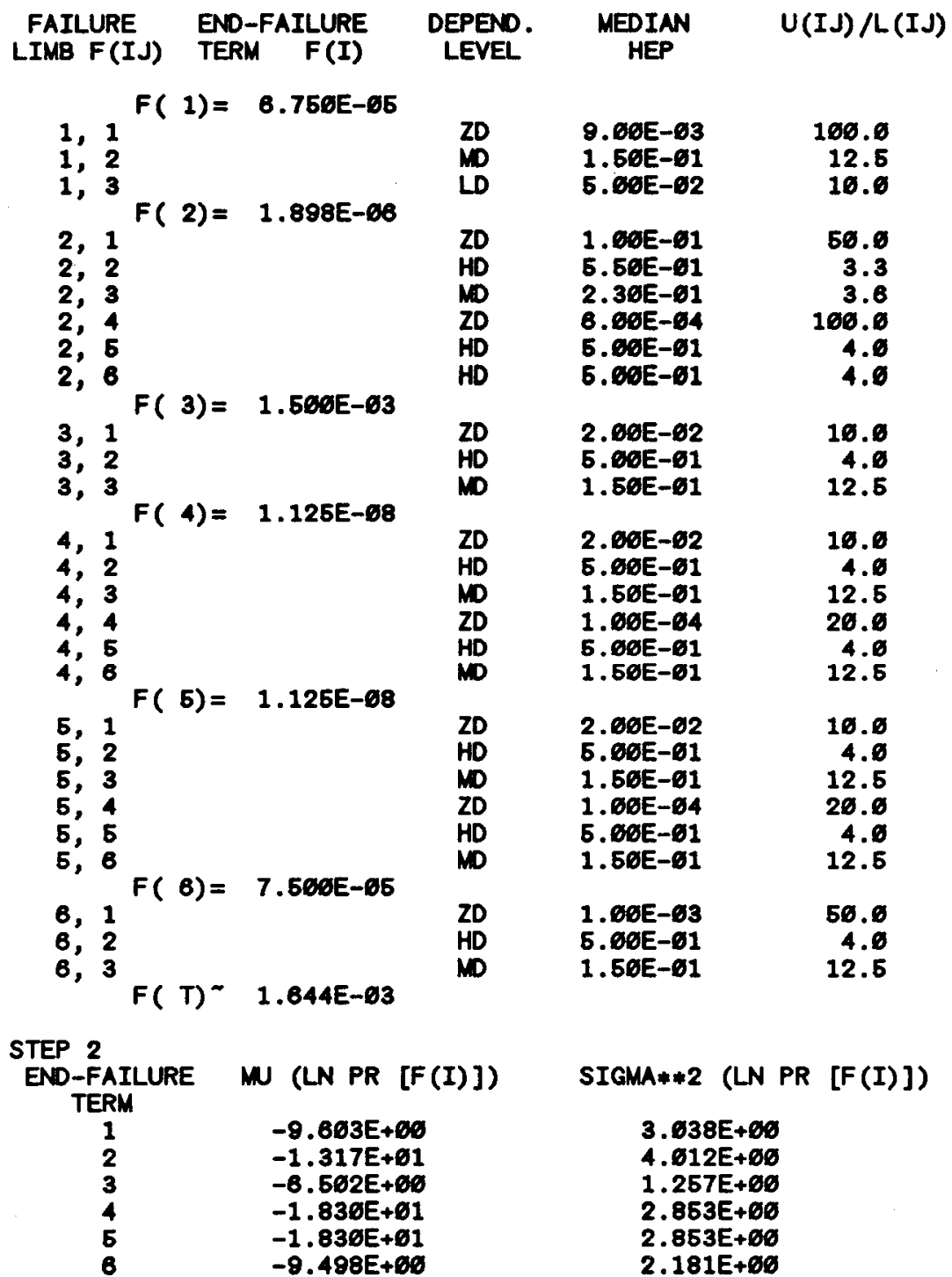

\begin{tabular}{|c|c|c|}
\hline $\begin{array}{l}\text { STEP } 3 \\
\text { END-FAILURE }\end{array}$ & MU (PR $[F(I)])$ & SIGMA**2 (PI \\
\hline $\begin{array}{l}1 \\
2 \\
3 \\
4 \\
5 \\
6\end{array}$ & 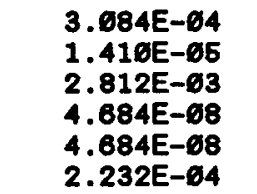 & $\begin{array}{l}1.896 \mathrm{E}-\varnothing 6 \\
1.678 \mathrm{E}-\varnothing 8 \\
1.988 \mathrm{E}-\varnothing 5 \\
3.584 \mathrm{E}-14 \\
3.584 \mathrm{E}-14 \\
3.911 \mathrm{E}-\varnothing 7\end{array}$ \\
\hline
\end{tabular}

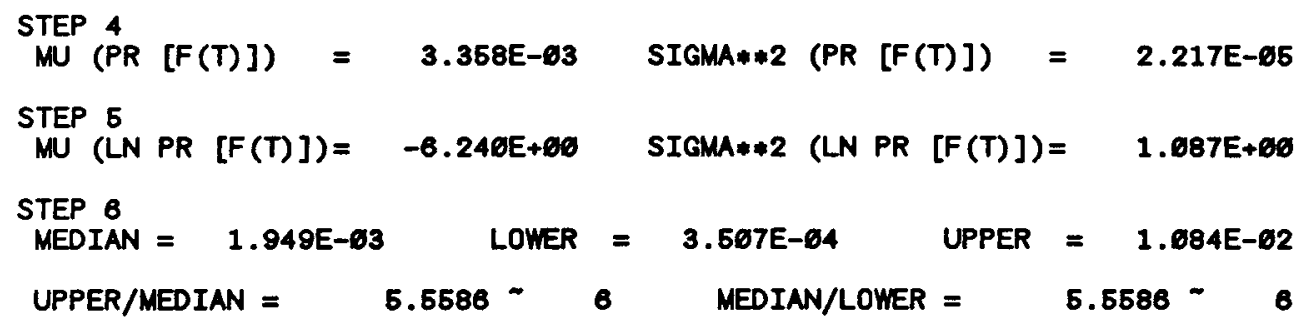


Table B-4 Computer Program for Program UCBs-Propagation ( $1 / 4$ )

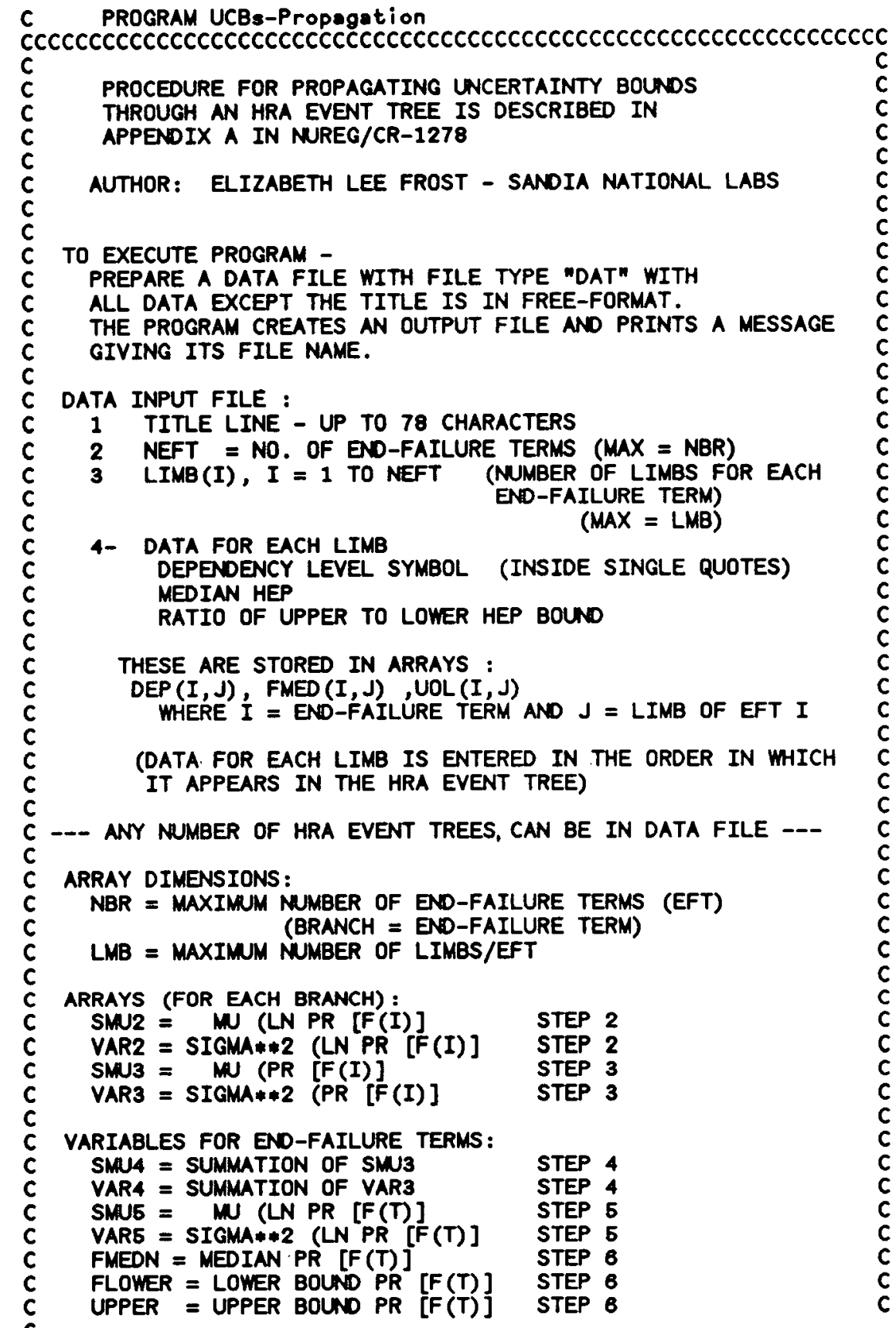


Table B-4 Computer Program for Program UCBs-Propagation ( $2 / 4$ )

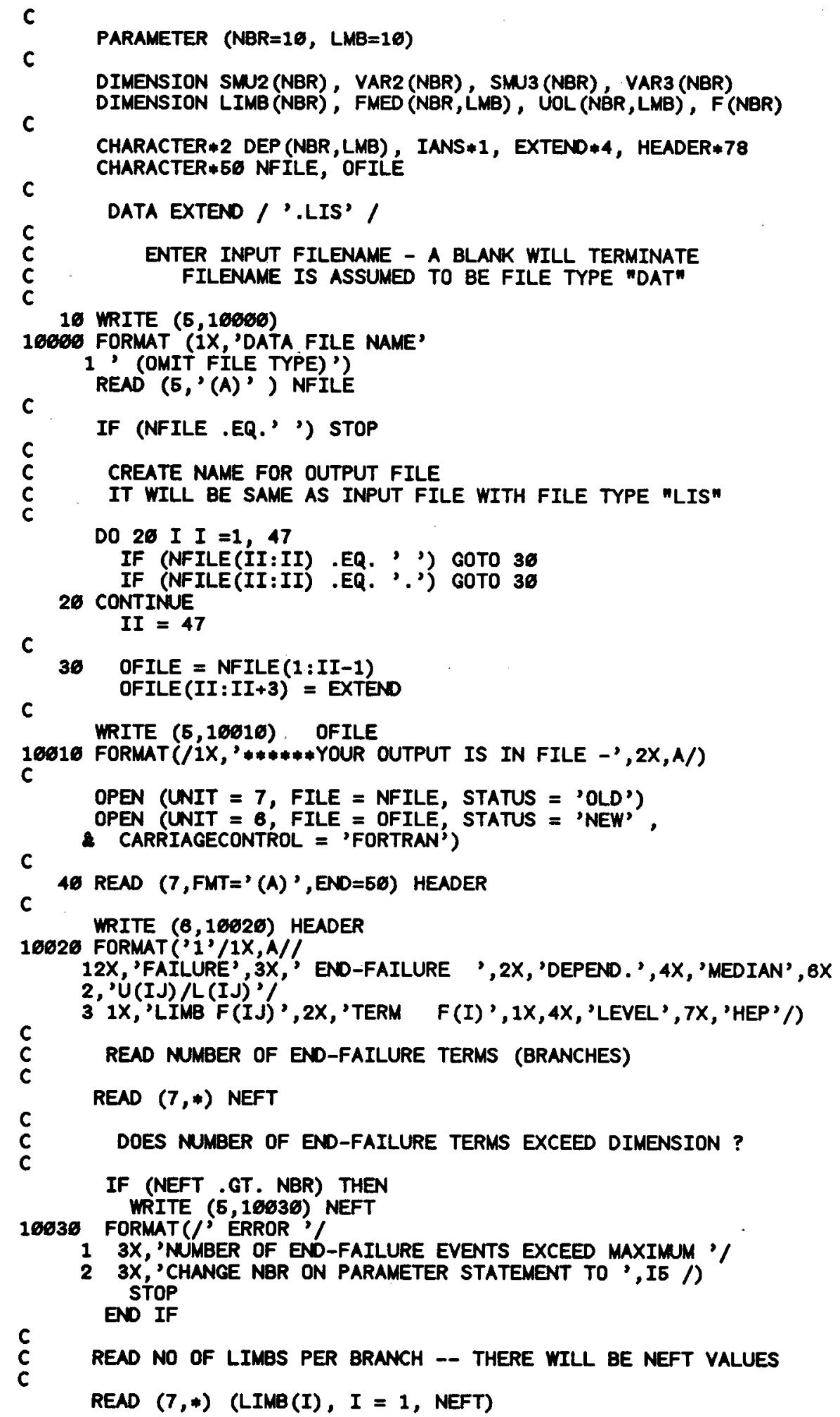


Table B-4 Computer Program for Program UCBs-Propagation (p3/4)

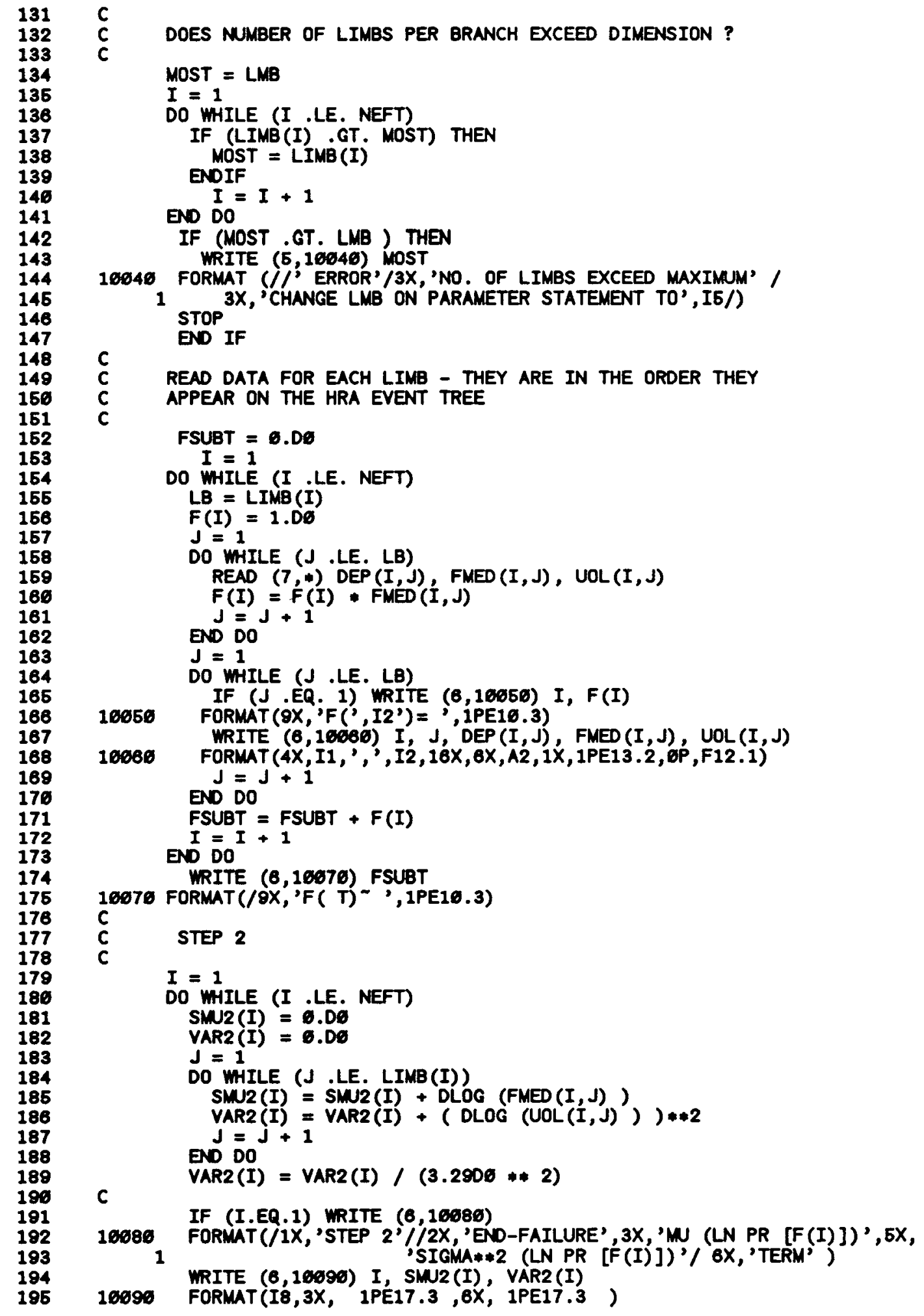


Table B-4 Computer Program for Program UCBs-Propagation (p4/4)

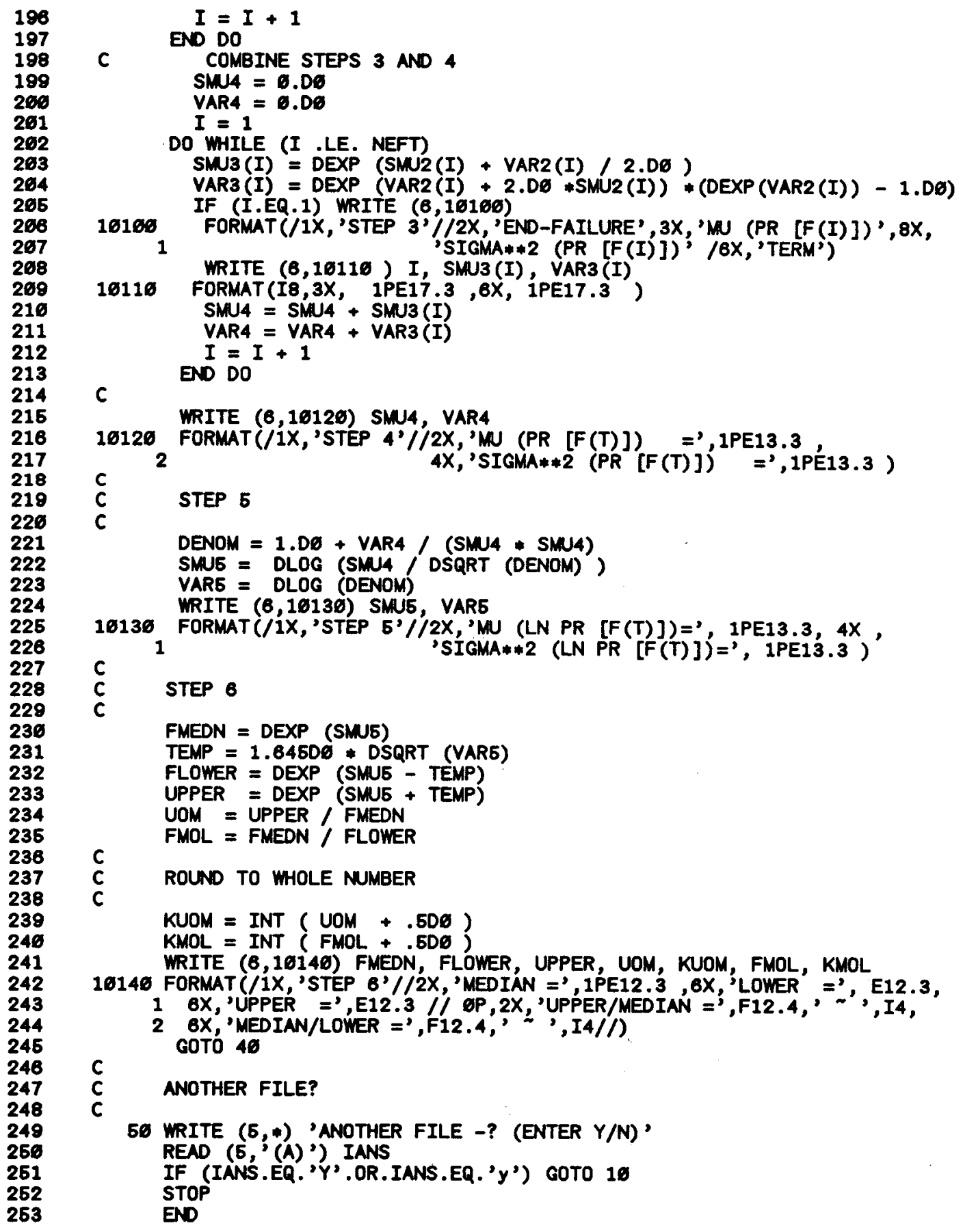


APPENDIX C. CORRECTIONS TO NUREG/CR-1278 AND NUREG/CR-2254

The ASEP HRA Procedure is based in large part on the final version of NUREG/CR-1278 (Swain and Guttmann, 1983) and refers to the human reliability analysis (HRA) methods described in the final version of NUREG/CR-2254 (Bell and Swain, 1983). Since the publication of these documents, several errors have been noted, and are listed in this appendix. Please notify A. D. Swain, 712 Sundown Place SE, Albuquerque, NM 87108, phone: (505) 265-0098 or 25507003, of any other major errors.

The above two documents may be purchased from: U.S. Government Printing Office, PO Box 37082, Wash. DC 20013-7082, phone: (202) 275-2060. The prices, including mailing costs, for NUREG/CR-1278 are: $\$ 26.00$ in the U.S.; $\$ 32.50$ by surface mail outside the U.S.; and $\$ 37.50$ by airmail outside the U.S. The prices, including mailing costs, for NUREG/CR-2254 are: $\$ 5.50$ in the U.S.; $\$ 6.88$ by surface mail outside the U.S.; and $\$ 11.88$ by airmail outside the U.S.

\section{Addendum \#1 to NUREG/CR -1278 , August 1983}

Following are some corrections to the above document, listing the page ( $p$ ) and paragraph (para) numbers. Understandable typos are excluded. Some updating of references is included. These changes were published by the U.S. Nuclear Regulatory Commission on September 1, 1985. Additional changes since that date are those on are indicated with a + sign.

\section{p,para \# Description of Corrections}

3-14,5 Line 3: ... are doubled for step-by-step tasks and quintupled for dynamic tasks. However, this

Line 6: ... of human reliability, e.g., an additional factor of 2 increase in the estimated HEP. This is an

[For further explanation, see the change for $p$ 17-7.]

3-35,5 Line 4: ...is generally correct, and is known as the Inverted-U Hypothesis or the Yerkes-Dobson Law (Welford, 1974; Fitts and Posner, 1967). This means ...

6-3,5 Line 7: Beare, Dorris et al, 1982, 1983). No ...

6-5,6 Line 6: (1984) under ...

7-15 Table 7-3, item (1)(a): .01 ( $E F=3,5$, or 10)

[This addition agrees with Table 7-2 which indicates conditions when any one of these EFs is relevant.]

11-8 Table 11-2 title, 1ine 2: ... displays (or annunciated displays no longer annunciating) for ...

11-16 Table 11-4, item (7): The ** should be followed by the same "cross" footnote symbol used for item (8). 
11-51 Table 11-13, add to last footnote: The $\overline{\operatorname{Pr}\left[F_{i}\right]}$ column assumes that all of the ANNs (or completely dependent sets of ANNs) are equal in terms of the probability of being noticed. See page 11-52, paragraph 2, if this assumption does not hold.

12-8,4 Line 7: .. emergency operating procedures...

12-12,3 Line 4\&5: ... Wreathall (1981, p 104, and 1982), ..

12-13 Figure 12-3: The 3 lines in the figure should meet at the $1.0 \operatorname{Pr}[F]$ rather than at the .9 HEP. Also, the 1 (e.g., HEP of 1.0) is incorrectly placed; it should be in the same relative position above the $.9 \mathrm{HEP}$ as is the .1 HEP above the $.09 \mathrm{HEP}$. The $\operatorname{Pr}[\mathrm{F}]$ values for the lower bound and median HEPs at 10 minutes are correct. The $\operatorname{Pr}[F]$ values for the upper bound HEP at 20 minutes should be 1.0 .

12-15+ Table 12-2: Change second footnote to read as follows: For points in between the times shown, use the medians and EFs from Figure 12-3 for the first event, and interpolate between the tables values for the second event.

12-17+ Table 12-3: Add the following to the table:

Exception (addition to original table):

(4) Failure to perform an immediate emergency action for $\quad .01 \quad 5$ the reactor vessel/containment critical parameters when (a) it can be judged to have been committed to memory, (b) it can be classified as skill-based actions, and (c) there is a backup written procedure

12-19+ Table 12-4: Change the second footnote to read as follows: For points in between the time shown, use the medians and EFs from Figure 12-4 for the first event, and interpolate between the tabled values for the second and third events.

12-20 Table 12-4: The 3 lines in the figure should meet at the $1.0 \operatorname{Pr}[F]$ rather than at the .9 HEP. Also, the 1 (e.g., HEP of 1.0) is incorrectly placed; it should be in the same relative position above the $.9 \mathrm{HEP}$ as is the $.1 \mathrm{HEP}$ above the $.09 \mathrm{HEP}$. The $\operatorname{Pr}[\mathrm{F}]$ values for the lower bound and median HEPs at 10 minutes are correct. The $\operatorname{Pr}[F]$ values for the upper bound HEP at 10 minutes should be 1.0 . 
13-6 Table 13-3: Items (2), (3), and (4) correctly pertain to the potential error, "Select wrong control on a panel from an array of similar-appearing controls." There should have been another item comparable to item (1) in Table 11-2 (p 11-8). In order not to change the item numbers in Table 13-3, insert the following item after item (1):

(1A) Select wrong control when it is Negligible dissimilar to adjacent controls

17-7 Footnote, line 3: ... multiplied by an additional factor of 2 because ...

[The intent of this change is to enable an analyst to apply the factor of 2 for step-by-step tasks or 5 for dynamic tasks, as discussed in paragraph 6 for "heavy task loading," and then to further increase the resultant $\mathrm{HEP}$ by an additional factor of 2 for the extra stressors listed in the footnote.]

19-4+ Table 19-1, paragraph preceding item (5): remove the .55 opposite the first line in the paragraph

19-4 Table 19-1, item (6): Rising stem with or without a position indicator*x

[This change is based on the conservative assumption that the checker will just look at the rising stem even when there is a position indicator.]

20-3 Figure 20-1 (p1 of 3): Insert a "Screening Required?" hexagon in the No line following the "Abnormal Event?" hexagon. The YES and NO lines from the new hexagon go to the "Rule-Based Actions?" hexagon. Insert a "Decide on Screening Rules" box in the YES Iine.

[This change is necessary because many PRAs require some form of screening for pre-accident situations.]

20-4 Figure 20-1 (p2 of 3), "Other PSFs" box: Other PSFs (see text, especially pp $3-14,-17,-19,-72$, and 17-7)

20-6,5 Item (2), line 7: ... Assume YES. (Note: Screening may also be required for non-abnormal tasks, as shown in the NO path following the ABNORMAL EVENT? hexagon.)

20-17+ Table 20-1: See change for $\mathrm{p}$ 12-15.

20-18+ Table 20-2: See change for $p$ 12-17

20-19+ Table 20-3: See change for p 12-19.

20-25 Table 20-9 title, line 2: ... displays (or annunciated displays no longer annunciating) ...

20-27 Table 20-11, item (7): The $* *$ should be followed by the same "cross" footnote symbol used for item ( 8 ). 
20-28 Table 20-12: Items (2), (3), and (4) correctly pertain to the potential error, "Select wrong control on a panel from an array of similar-appearing controls." There should have been another item comparable to item (1) in Table 20-9 (p 20-25). In order not to change the item numbers in Table 20-12, insert the following item after item (1):

(1A) Select wrong control when it is Negligible dissimilar to adjacent controls

20-37 Table 20-21, item (1)(a): .01 ( $E F=3,5$, or 10)

[This addition agrees with Table 20-20 which indicates conditions when any one of these EFs is relevant.]

20-38+ Table 20-22, paragraph preceding item (5): remove the .55 opposite the first line in the paragraph

20-38 Item (6) in Table 20-22: Rising stem with or without a position indicator**

[This change is based on the conservative assumption that the checker will just look at the rising stem even when there is a position indicator.]

20-39 Table 20-23, add to last footnote: The $\overline{\operatorname{Pr}\left[F_{i}\right.}$ column assumes that all of the ANNs (or completely dependent sets of ANNs) are equal in terms of the probability of being noticed. See page 11-52, paragraph 2, if this assumption does not hold.

20-44 See change for $\mathrm{p} \mathrm{20-3.}$

20-45 See change for $\mathrm{p} \mathrm{20-4.}$

22-5,1 Line 11: Beare, Dorris et al, 1982, 1983).

A-9 Step 5, the numerator in the first equality: $\mu_{\operatorname{Pr}\left[F_{\mathrm{T}}\right]}$

[The $\mu$ was left out.]

H-2 7th reference, line 5: DC, January 1984.

H-2 9th reference, line 3: ... New York: Plenum Press, 1984

H-3 9th reference, line 4: ... and M. W. McCann, Review and Evaluation of the Zion Probabilistic Safety Study, Sandia National Laboratories, ... DC, January 1984.

H-4 6th reference, line 1: ... Kozinsky, D. S. Crowe, ..

H-5 2nd \& 3rd references: Add "Inc." after "Human Performance Technologies,".

H-13 4th reference, line 3: ... and D. M. Kunsman, ... 
H-15 13th reference, line 2: .. Society, 1984.

H-15 14th reference, line 3: ... Press, 1984.

H-16 4th reference, line $1: \ldots$ Comments on Draft NUREG/CR-1278, . . line 2: ... NM, May 1985 .

H-17 10th reference, line 2: Vols 1 and $2, \ldots$

H-18 4th reference, line 2: Society and the Institute...

H-21 8th reference, line 3: Nuclear Safety, ...

H-22 4th reference, lines $3 \& 4:$...Laboratory, NUREG/CR-3114, U.S. Nuclear Regulatory Commission, Washington DC, December 1982.

H-22 6th reference, line 5: DC, August 1983.

H-25 6th reference, line 2: .. Equipment, D\&A-TR-60-36F, ...

H-26 6th reference, line 4: Press, 1984.

J-7 3rd definition, line 1: ... part of diagnosis: ..

line 2: ... alternative diagnoses, ... [remove underlining]

J-8 7th definition, line 2: .. identify those systems ...

J-8 14th definition, line 2: ... level in Tank A ...

J-16 10th definition, line 1 etc: . . of human error on all of the tasks in the complete-failure path.

J-16 11th definition, line 1 etc: ... of no human errors on all of the tasks in the complete success path. 


$$
\mathrm{C}-6
$$

\section{Addendum \#1 to NUREG/CR-2254, May 1983}

Following are some corrections to the above document, listing the page ( $p$ ) and place on the page to be corrected.

p 21, Step D.2, next-to-last line: Change values to valves

p A-18, column labeled 6. Table No. \& Item No.: Change 20-8, \#1 to 20-8, \#1a

p A-18, column labeled 7. Tabled HEP and UCBs: Change .01 (.003 to .03) to .01 (.002 to .05)

p A-20, line 2: Change .014 to .06

p A-44, failure limb labeled $B$ in the figure: Change 0.1 to .01

p A-52, line 2: Change $10^{5}$ to $10^{-5}$

pp A-54, A-55: When we revised NUREG/CR-2254 to its May 1983 version, we forgot to change failure limbs $A$ and $H$. For $A$, the present document incorrectly refers to $\mathrm{T} 20-8$, the table for oral instructions. Using the search scheme (Ch. 20 in NUREG/CR-1278), for omission errors and assuming written materials, we should consider errors in preparation of written materials (T20-5 from NUREG/CR-1278), implementation of administrative control (T206), and use of written procedures (T20-7). Since the problem assumes a Leve1 1 tagging system (T20-15), it is likely that there are sufficient checks on the accuracy of a restoration procedure and that errors in the procedure itself can be presumed to be negligible. To further simplify the problem, we assume that written procedures are used (rather than relying on memory) and that the restoration tasks are performed. Therefore, only T20 7 is relevant to the problem. Assuming no more than 10 valves must be restored and that the restoration procedure does not incorporate a checklist, item \#3 in $\mathrm{T} 20-7$ is relevant. The nominal HEP is .003, but since Level 1 tagging is used, item \#1 in T20-15 shows that we should use the lower bound for the $.003, \mathbf{i} . \mathrm{e}, .001$. So we are back to the original .001 in the problem.

For failure limb $\mathrm{H}$, the wrong HEP and source are used. Using T-20-11, \#8. the HEP is negligible ( $\epsilon=$ epsilon, the usual PRA abbreviation for "negligible."). This change would obviously reduce the $F_{T}$ in Figure $A-22$ to $<10^{-5}$ (usually stated as $<1 \mathrm{E}-5$ in PRAs). 


\section{DISTRIBUTION}

Government Printing Office

U.S. NRC Distribution Contractor

Receiving Branch (Attn: NRC Stock)

8610 Cherry Lane

Laure 1, MD 20707

320 copies for $A N, R X$

Dr. Shahid Ahmed

Be11 Communications Research

Mail Stop NVC 2X-347

331 Newman Springs Rd.

P.O. Box 7020

Red Bank, NJ 07701

Prof. Agustin Alonso Santos

Jose Gutierrez Abascal, 2

2800 Madrid

Spain

R. L. Anderson

AT\&T, Room 2EC123

1 Oak Way

Berkeley Heights, NJ 07922-2727

Dr. Kiyoji Asai

University of Osaka Prefecture

Mozu, Umemachi

Sakai, Osaka 591

Japan

Stuart Asselin

International Energy Associates, Ltd.

1701 Louisiana, NE, Suite 202

Albuquerque, NM 87110

Ruta Axelsson

LUTAB

P. O. Box 52

S-161 26 Bromma

Sweden

Dr. Werner Bast1

GRS - Bereich Systeme

Forschungsgel ande

8046 Garching

Federal Republic of Germany
Roger J. Batstone

Office of Environmental and

Scientific Affairs

World Bank (Room D-1011)

$1818 \mathrm{H}$ Street, NW

Washington, DC 20433

Dr. Paul Baybutt, President

Technica Inc.

355 E. Campus View Blvd.

Suite 110

Columbus, $\mathrm{OH} \quad 43085$

David Beattie

Ontario Hydro H-14

700 University Ave.

Toronto, ON M5G 1X6

Canada

B. J. Bell

Risk, Safety, and Reliability

Analysis Section

Battelle Columbus Division

505 King Ave.

Columbus, $\mathrm{OH} 43201$

Robert Bertucio

Energy Inc.

1851 S. Central Place

Suite 201

Kent, WA 98031

C. J. E. Beyers

Licensing Branch (Standards)

Atomic Energy Board

Private Bag X256

Pretoria 0001

Republic of South Africa

Dr. Robert E. Blanchard

M-8 Monticello St.

Del Mar, CA 92014

Dennis C. Bley

PL\&G

2260 University Drive

Newport Beach, CA 92660

Dr. C. A. Bolig

GA Technologies, Inc.

P.O. Box 85608

San Diego, CA 92138 
Terry F. Bott

Los Alamos National Laboratory

Group Q6, Mail Stop K557

P. O. Box 1663

Los Alamos, NM 87545

R. F. Bradley

NRSED

Savannah River Lab

Aiken, SC 29808

Frank Briscoe

Safety and Reliability Directorate

UKAEA

Wigshaw Lane, Culcheth

Warrington WA3 4NE, Cheshire

England

Dr. Robert Budnitz

Future Resources Associates Inc.

2000 Center St., Suite 418

Berkeley, CA 94704

William M. Burch

Office of Toxic Substances

U.S. Environmental Protective Agency

501 M Street, SW

Washington, DC 20460

Lennart Carlsson

Swedish Nuclear Power Inspectorate

Box 27106

10252 Stockholm, Sweden

Thomas W. Carmody

Center for Chemical Process Safety

AIChE

345 E. 47th Street

New York, NY 10017

Annick Carnino

3 Avenue de Fauvettes

91400 Orsay, France

Dr. Raphael Caro Munso

Dept. de Seguridad Nuclear

Av. Complutense, 22

Madrid 3 Spain
Nathan Cathey

EG\&G Idaho

TSB Building

Idaho Falls, ID 83415

Per Cederholm

FMV: FLYSEL

S-11588 Stockholm

Sweden

Dr. Julien M. Christensen

5950 Little Sugar Creek Rd.

Dayton, $\mathrm{OH} 45440$

Bob Christie

Availability and Reliability Section

Nuclear Engineering Branch

Tennessee Valley Authority

W10C126

400 Commerce Ave.

Knoxville, TN 37902

Dr. Lloyd Collins

Emerson Electric

Electronics and Space Division

Station 4076

8100 W. Florissant Ave.

St. Louis, MO 63136

Ken Corbitt

Carolina Power \& Light

QA Trailer

P.O. Box 790

Hartsville, SC 29550

Dr. E. N. Corlett

Editor, Applied Ergonomics

Dept. of Production Eng. and Prod.

Management

University of Nottingham

University Park

Nottingham NG7 England

Dr. Vincent T. Covello

Office of Scientific, Technical, and International Affairs

National Science Foundation

1800 G Street, NW

Washington, DC 20550 
Dr. R. A. Cox

Technica Ltd.

Lynton House

7112 Tavistock Square

London WC1 9LT England

Dr. Michael Cullingford

International Atomic Energy Agency

P. 0: Box 100

A-1400 Vienna

Austria

Orville Cypret

Arkansas Power \& Light Co.

General Research \& Development

P. O. Box 551

Little Rock, AR 72203

G. Dah1

Norsk Hydro

P.O. Box 110

N-3901 Porsgrunn

Norway

Alain Debiar

867, Cours Aquitaine

92100 Boulogne

France

Det norske Veritas

Human Factors (Egonomics)

P.O. Box 300

$\mathrm{N}-1322$ H $\phi \mathrm{vik}$

Oslo, Norway

Dr. David Dinges

Unit for Experimental Psychiatry

$111 \mathrm{~N} 49$ th St.

Philadelphia, PA 19139-2798

M. T. Drouin

SAIC

2109 Air Park Rd.

Albuquerque, NM 87106

W. S. Durant

Actinide Technology Div.

Savannah River Lab

Aiken, SC 29808-0001
Dr. Elwyn Edwards

Dept. of Applied Psychology

University of Aston

Birmingham B4 7EK

England

Dr. David Embrey

Human Reliability Associates, Inc.

1 School House

Higher Lane, Dalton, Parbold

Lancaster WN8 7RP

England

Ente Nazionale per L'Energia

Elettrica

Centro Ricerca de Automatica

ENEL - CRA

Via Valv, Peroni, 77

20133 Milano, Italy

Dr. Ralph A. Evans

Editor, IEEE Transactions on Rel.

804 Vickers Avenue

Durham, NC 27701

L. Felkel

GRS - Bereich Systeme

Forschungsgelande

8046 Garching

Federal Republic of Germany

Rene A. Fernandez Gonzalez

Dept. de Ing y Projectos

Centrales Nucleares del Norte, S.A.

H. Cortes, 57

Santender, Spain

Randy Fisher

Atomic Energy of Canada Ltd.

Sheridan Park Research Community

Mississauga, Ontario L5K $1 \mathrm{BZ}$

Canada

Dr. John D. Folley, Jr.

Applied Science Associates

Box 158

Valencia, PA 16059

Joseph R. Fragola

SAIC

274 Madison Ave., Suite 1501

New York, NY 10016 
Haruo Fujimoto

Mitsubishi Atomic Power Ind. Inc. 4-1, Shibakouen 2-Chome

Minato-ku, Tokyo 105

Japan

Prof. Dr. I. Fukuyama

Dept. of Safety Engineering

Yokohama National University

156 Tokiwadai, Hodogaya-ku

Yokohama 240

Japan

Dr. J. B. Fusse11

JBF Associates, Inc.

Technology Drive

1000 Technology Park Center

Knoxville, TN 37922

Bernard Gachot

Electricite de France

Region de Equipement

Alpes-Marseille

140, Avenue Viton - B.P. 560

13275 Marseille Cedex 9

France

\section{Paul Gagnolet}

Electricite de France

Service de la Production Thermique

Department Surete Nucleaire

71 rue Miromesnil

F75008 Paris

France

J . P. Garde

26 rue diaz

33000 Bordeaux

France

Dr. B. John Garrick

Pickard, Lowe \& Garrick, Inc.

2260 University Drive

Newport Beach, CA 92660

J. P. Gayral

20 Avenue Fructidor

33115 Pyla Sur Mer

France
S. B. Gibson

Engineering Service Division

Engineering Department

E. I. du Pont deNemours \& Co.

Wilmington, DE 19898

Robert Gilbert

Dept. of Genie Industriel

Ecole Polytechnique

Campus Universite de Montreal

C.P. 6079, Succ. A

Montrea1 PQ H3C $3 \mathrm{~A} 7$

Canada

Dr. Timothy Goldsmith

Department of Psychology

University of New Mexico

Albuquerque, NM 87131

Prof. Etienne P. Grandjean

Department of Ergonomics

Swiss Federal Institute of Technology

21 Clausius St.

8092 Zurich $\mathrm{CH}$

Switzerland

Martine Griffon-Fouco

Electricite de France

Service de la Production Thermique

71, Rue de Miromesnil

75008 Paris

France

Hans Jorgen Grundt

Statoil, P.0. Box 300

N-4001 Stavanger

Norway

Hasim Gundagdu

Operations Research Division

Marmara Scientific and Industrial

Research Institute

P.O. Box 21

Gebze-KOCAELI

Turkey

H. E. Guttmann

3904 Wellesley NE

Albuquerque, NM 87107 
Pilsoon Han, Ph.D.

Korea Advanced Energy Research Inst.

P.0. Box 7, Cheong Ryang

Seoul, Korea

Dr. G. W. Hannaman

NUS Corp., Suite 250

16885 W. Bernardo Drive

San Diego, CA 92127 .

Dr. Douglas H. Harris

Anacapa Sciences, Inc.

P.O. Drawer Q

Santa Barbara, CA 93102

Tosaku Hattori

Industrial Designer

Naka Works

Hitachi Ltd.

882 I Chige

Katsuta, Ibaraki, 312

Japan

Prof. Yoshio Hayashi

Dept. of Administration Engineering

Keio University

3-14-1 Hiyoshi, Kohoku

Yokohama 223

Japan

Matti Heikkila

Institute of Radiation Protection

Department of Reactor Safety

P.O. Box 268

00202 Helsinki 10

Finland

Tor Heimly

Det norske Veritas

$300, \mathrm{~N}-1322 \mathrm{H} \phi \mathrm{vik}$

Norway

Dr. O. H. Helles $\phi y$

Mobil Exploration Norway Inc.

Kokstadflaten 9

5065 Blomsterdalen, Norway

C. Hensley

British Nuclear Fuels, Ltd.

Risley, Warrington

England WA3 6AS
Risto Himanen

TVO

27160 Olkilvotn, Finland

E. M. Hinchley

Reactor Control and Safety Systems

Atomic Energy of Canada

Sheridan Park Research Community

Mississauga, ON L5K 1B2

Canada

Prof. Fritz Hjelte

Dept. of Aeronautics

The Royal Institute of Technology

S-100 44 Stockholm 70

Sweden

David L. Honkonen

Building 320M

Savannah River Plant

Aiken, SC 29808

Dr. Helmut Hortner

Systems Analysis Section

Gesellschaft fur Reaktorshicherheit (GRS) $\mathrm{mbH}$

Forschungsgelande

8046 Garching

Federal Republic of Germany

Nial1 Hunt

Baltimore Gas \& Electric

P.O. Box 1475

Fort Smallwood Road Complex

Baltimore, MD 21203

Dr. D. Ilberg, Head

Systems Safety and Accident

Analysis Dept.

Licensing Division

Atomic Energy Commission

P.O. Box 17120

Te1-Aviv

61070 Israel

Rick Imig

Shell Oil Co.

P.O. Box 100

Deer Park, TX 77536 
Dr. Tetsuro Itakura, Director

Engineering Department

The Japan Atomic Power Co.

Ohtemachi Building

$6-1,1$-Chome, Ohtemachi

Chiyoda-ku, Tokyo 100

Japan

Mutsumi Itoh

Contro1 \& Electrical Engineering Dept.

Nuclear Energy Group

Toshiba Corporation

13-12, MITA 3-Chome, Minato-ku

Tokyo 108

Japan

William G. Johnson

151 Shelter Cove Drive

Half Moon Bay, CA 94019

Ulf Jonsson

OKG Aktiebolag

Box 1746

S-111 87 Stockholm, Sweden

Dr. Peter Kafka

Gesselschaft fur Reaktorsicherheit (GRS) $\mathrm{mbH}$

Forschungs gelande

8046 Garching

Federal Republic of Germany

Dr. Stanly Kaplan

Pickard, Lowe \& Garrick

220 University Drive

Newport Beach, CA 92660

Dr. Gyorgy Karmos

Institute of Psychology

Hungarian Academy of Science

Budapest, Hungary

Mardyros Kazarians

Pickard, Lowe \& Garrick

220 University Drive

Newport Beach, CA 92660

Larry Kirkland

Human Resources Department

Westinghouse Nuclear Fuel Division

P.O. Drawer R

Columbia, SC 29250
Prof. Trevor A. Kletz

64 Twiny Brook Road

Cheadle Hulme

Cheadle, Cheshire SK8 5RJ

England

George Klopp

Commonwealth Edison

Station Nuclear Engineering

Room 35W

P.0. Box 767

Chicago, IL 60690

Dr. William B. Knowles

Psychology Department

California State Univ., Northridge

Northridge, CA 91330

Will Kochinski

Loss Prevention Department

Technical Services, E-1855

Aramco

Dhahran, Saudia Arabia

A. M. Kolaczkowski

SAIC

2109 Air Park Road

Albuquerque, NM 87106

Dr. Jefferson M. Koonce

Lead Mine Hill Road, RFD \#3

Amherst, MA 01002

Donald W. Latham

San Diego Gas and Electric

P.O. Box 1831

San Diego, CA 92112

Duk-Sun Lee

Nuclear Training Center

Korea Advanced Energy Research Inst.

P.0. Box 7, Cheong Ryang

Seoul, Korea

Dr. Frank P. Lees

Department of Chemical Engineering Loughborough University of Technology Loughborough, Leics, LE11 3TU

England 
Fernand Leonard

Chef du Departement BR2

Centre d'Etude de 1'Energie Nucleaire

Boeretang 200

B-2400 Mo1, France

Prof. Hal Lewis

Physics Department

University of California

Santa Barbara, CA 93106

Pierre M. Lienart

Electricite de France

Thermal Generation Division

OP-NS Department

3 , Rue de Messine

75384 Paris Cedex 08, France

Lars G. Linden

Scandinavian Seminars $\mathrm{AB}$

S-340 30 Vislanda

Sweden

Bo L1wang

Swedish Nuclear Power Inspectorate

Box 27106

S-10252 Stockholm

Sweden

D. K. Lorenzo

JBF Associates

Technology Drive

1000 Technology Park Center

Knoxville, TN 37922

William J. Luckas, Jr.

Nuclear Safety Programs

Building 130

Brookhaven National Lab

Upton, NY 11973

LUTAB

Attn: Library

P.0. Box 52

S-161 26 Bromma

Sweden

James F. Mallay

Nuclear Safety Analysis Center

EPRI

P.O. Box 10412

Palo Alto, CA 94303
Dr. Thomas Mankamo

c/o Prof. Bjфrn Wahlstr $\phi \mathrm{m}$

Electrical Engineering Lab

Tech. Research Centre of Finland (VTT)

Otakaari 7B ESPOO 02150

Finland

Dr. D. J. Martin

Atomic Energy Control Board

P.0. Box 1046

Ottawa, Canada

KIP 559

Steve Mays

Delian Corp.

545 Shoup Ave., Suite 310

Idaho Falls, ID 83402

Dr. M. Mazumdar

Dept. of Industrial Engineering

University of Pittsburgh

Pittsburgh, PA 15260

T. W. McClelland

Loss Prevention Dept.

Aramco Box 55

Dhahran, Saudi Arabia

Jean-Jacques Mira

Electricite de France

Serice de la Production Thermique

71, Rue de Miromesnil

75008 Paris, France

Mitsubishi Heavy Industries Ltd.

Pittsburgh Representatives Office

c/o Westinghouse Electric Corp.

Penn Center Bldg. 3, Room 600

Pittsburgh, PA 15230

Prof. Richard A. Moll

Dept. of Eng. \& Applied Science

University of Wisconsin

432 North Lake St.

Madison, WI 53706

Dr. Kazuo Monta

Nuclear Engineering Dept.

NAIG Nuclear Research Lab.

4-1, Ukishima-cho Kawasaki-ku

Kawasaki-shi, Kanagwa-ken

210 Japan 
David Moore

Energy Inc., Suite 201

1851 S. Central Place

Kent, WA 98031

Prof. J. Moraal

Institute for Perception

Soesterberg

Kampweg 5, Postbus 23

Holland

Jack Mott

Advanced Reactor Systems Dept.

S- 65

General Electric Co.

P.O. Box 3508

Sunnyvale, CA 94088

G. Richard Mullee

Nuclear Services Dept.

General Electric Co.

175 Curtner Ave., M/C 853

San Jose, CA 92125

Phillipe Namy

Probabilistic \& Risk Anal. Gp.

Framatome S.A.

Tour Fiat

1 Place de la Coupole

F-92084 Courbevoie, Cedex 16

France

Mohammad Nasim

Nuclear Safety \& Licensing Div. Pakistan Atomic Energy Comm.

P.O. Box 1114

Islamabad, Pakistan

Dr. David Navon

University of Haifa

Mount Carmel, Haifa 31999

Israel

Larry Noyes

Philadelphia Electric Co.

2301 Market St., S12-1

Philadelphia, PA 19101

Nuclear Safety Research Association

P. O. Box 1307

Falls Church, VA 22041
Dr. John N. O'Brien

Nuclear Safety Program, Bldg. 130

Brookhaven National Lab

Upton, NY 11973

Reider $\varnothing$ stvik

SINTEF

N7034 Trondheim

NTH

Norway

Magnus $\emptyset$ vreide

Institutt for Energiteknikk

OECD Halden Reactor Project

P.O. Box 173

N-1751 Halden

Norway

Dr. Ray Parsick

Safeguards Evaluation Section

International Atomic Energy Agency

Wagrannerstrasse 5, P.0. Box 100

A-1400, Vienna

Austria

Kathleen Paul

Carolina Power \& Light

Fayetteville Street Mal1, Suite 200

Wake County Office Bldg.

Raleigh, NC 27602

John Payne, Editor

Nuclear News

American Nuclear Society, Inc.

555 N. Kensington Avenue

La Grange Park, IL 60525

Dr. Dominque Pignon

Centre National de la Recherche

Scientifique

Laboratoire de Physique Theoretique de 1'Ecole Normale Superieure

24, rue Lhomond 24

75231 Paris Cedex 05

France

Dr. E. C. Poulton

NRC Applied Psychology Unit

15 Chaucer Road

Cambridge, CB2 2EF

England 
Leonard C. Pugh, Sr.

General Electric Co.

Advanced Reactor Systems Dept.

P.O. Box 508

Sunnyvale, CA 94086

William Rankin

Battelle Human Affairs Research

Center

$4000 \mathrm{NE} 41$ st St.

Seattle, WA 98105

Prof. Jens Rasmussen

Sm $\phi$ rum Bygade 52

Dk-2760 Mal $\phi v$, Denmark

Dr. Norman Rasmussen

Nuclear Engineering Dept.

Massachusetts Institute of Technology

Cambridge, MA 02139

T. J. Ravishanker

Ontario Hydro

700 University Ave.

Toronto, ON M5G 1X6

Canada

Prof. J. T. Reason

Dept. of Psychology

University of Manchester

Manchester, M1P 93L

England

Gunter Reichart

GRS - Bereich Systeme

Forschungsgelande

8046 Garching

Federal Republic of Germany

Louis Restrapo

5704 West 109 th Place

Westminster, CO 80020

Dr. Kyou H. Rhyi

30, Gyurngi-Dong,

Jongro-ku

Seoul, Korea

Ausra Richards

NUS

4 Research Place

Rockville, MD 20850
Robert C. Roberts

Babcock \& Wilcox

P.O. Box 1260

Lynchburg, VA 24505

Prof. Dr. Jan Rosner

Polish Ergonomics Society

UL. Gornoslaska 20

00-484 Warszawa

Poland

Dr. William B. Rouse

1886 Vanderlyn Drive

Dunwoody, GA 30338

Bo Rydnert

Brahegatan 5

S11437 Stockholm

Sweden

Dr. Mark S. Sanders

Dept. of Psychology

California State University, Northridge

Northridge, CA 91330

Yusuke Sawaguchi

Nuclear Power Plant Operation and Maintenance Dept.

The Tokyo Electric Power Co.

No. 1-3, 1-Chome, Uchisaiwai-cho

Chiyoda-ku, Tokyo 100

Japan

Dr. Lothar Schroeder

Human Factors Group

UNC Nuclear Industries

P.0. Box 490

Richland, WA 99352

Dr. Ahmad Shafaghi

Technica Inc.

355 E. Campus View Blvd.

Suite 110

Columbus, $\mathrm{OH} 43085$

Science News

Behavioral Sciences Editor

1719 N Street, NW

Washington, DC 20036 
Prof. Dr. J. W. Senders

Kenegy West

Columbia Falls, ME 04623

Dr. Mildred Shaw

Centre for Man Computer Sciences

94 Shakespeare Tower

Barbican,

London EC2Y 8DR, England

Dr. David Shinar

Ben-Gurion Univ. of the Negev

Beer Sheva 84120

P.0. Box 653

Israel

Wataru Shinoda

Plant Management Dept.

Japan Atomic Power Co.

Othemachi Bldg.

6-1, 1-Chome, Othemachi

Chiyoda-ku, Tokyo 100

Japan

John Spencer

Book Reviews Editor, Ergonomics

Dept. of Applied Psychology

University of Wales

Inst. of Science and Technology

Penylan, Cardiff CP3 7UX, Wales

Dr. Desmond Stack

Group Q6, Mail Stop K-557

Los Alamos National Lab

Los Alamos, NM 87545

Dr. Michael Stamatelatos

GA Technologies

P.0. Box 81608

La Jolla, CA 92138

Dr. Michael E. Stephens

Nuclear Safety Division

OECD Nuclear Energy Agency

38, Boulevard Suchet

F-75016 Paris, France
Catherine Stewart

TRW Human Factors

Mail Stop 523/313

Norton AFB

San Bernardino, CA 92402

Ragnar Georg Stokke

Norsk Hydro

N-3901 Porsgrunn

Norway

Mitsuo Suzuki

Nuclear Power Division

The Federation of Power Companies

Keidanren Kai Kan Bldg.

9-4, 1-Chome, Ohte-Machi

Chiyoda-ku, Tokyo

Japan

Michael Sykos 191434

Aramco

Box 5771 Abgaig

Dhahran, Saudi Arabia

Dr. Atsushi Takeda

Tokai Nuclear Generating Station \#2

The Japan Atomic Power Co.

Tokai-mura, Ibaraki-ken

Japan

Dr. Toshihide Takesita

Inst. for Policy Services

Friend Bldg.

2-4-1I Nagata-cho, Chiyoda-ku

Tokyo 100

Japan

Akira Tanabe

Reactor Design Engineering Dept.

Nuclear Energy Group

Toshiba Corp.

Isogo Engineering Center

8, Shinsugita-cho, Isogo-ku

Yokohama 235

Japan

Tord Sterner

ASEA-ATOM

Box 53

S-721 04

Vasteras, Sweden 
Tomihiro Taniguchi

Nuclear Power Operation

Administration Office

Agency of Natural Resources

Ministry of International Trade and Industry

131 Kasumigaseki, Chiyoda-ku

Tokyo 100, Japan

Dr. David A. Thompson

Industrial Engineering and

Engineering Management

Stanford University

Stanford, CA 94305

Dr. Toshiaki Tobioka

Reactor Safety Code Dev. Lab

Div. of Reactor Safety Evaluation

Tokai Research Establishment, JAERI

Tokai-mura, Naka-gun, Ibaraki-ken

Japan

Dr. Donald A. Topmiller

1576 Burchwood Drive

Fairborn, $\mathrm{OH} \quad 45324$

Ryosuke Tsutsumi

Nuclear Planning Division

The Tokyo Electric Power Co.

No 1-3, 1-Chome, Uchisaiwai-cho

Chiyoda, Tokyo 100

Japan

Odd J. Tveit

Statoil

P.O. Box 300 Forus

4001 Stavanger

Norway

Hiroshi Ujita

Energy Research Laboratory

Hitachi Ltd.

1168 Moriyamacho, Hitachi,

Ibaragi 316 Japan

Prof. R. Uliana

Universite de Bordeaux 1

Institut Universitaire de

Technologie A

Dept. Hygiene et Securite

33405 Talence Cedex

France
Alfred Unione

Energy Incorporated

3375 Scott Blvd. Suite 320

Santa Clara, CA 95054

Dr. Jan Van Erp

Div. of Educational Programs

Argonne National Lab

9700 S. Cass Avenue

Argonne, IL 60439

G. Van Reijen

Commission of the European

Communities

Directorate General XII

202 Rue de la Loi

Brussels 1040

Belgium

G. B. Varnado

International Energy Assoc., Ltd.

1717 Louisiana Blvd., NE, Suite 202

Albuquerque, NM 87110

Dr. William E. Vesely, Jr.

SAIC

2929 Kenny Rd., Suite 245

Columbus, $\mathrm{OH} \quad 43221$

Dr. W. Vinck

Commission of the European

Communities

Directorate General XII

202 Rue de la Loi

Brussels 1040

Belgium

Prof. Guiseppe Volta

European Joint Research Center

ISPRA (VARESR)

Italy

Prof. Bjфrn Wahlstrom

Electrical Engineering Lab

Tech. Research Centre of Finland (VTT)

Otakaari 7B ESPOO 02150

Finland

Jiro Wakabayashi

12-19, Shugakuin-Kitafukecho

Sakyo-ku, Kyoto 606

Japan 
Dr. Ray Waller

Los Alamos National Lab

P.O. Box 1663

Los Alamos, NM 87545

I. A. Watson

UKAEA

Safety \& Reliability Directorate

Wigshaw Lane, WA3 4NE Cheshire

England

Dr. Meyer Weinstein

7550 Rainbow Drive

Cupertino, CA 95014

Røllf A. Westesson

LUTAB, Box 52

S-161 26 Bromma

Sweden

Dr. Walter W. Wierwille

Dept. of Industrial Engineering and Operations Research

VPI\&SU

Blacksburg, VA 24061

James R. Wilson

Safety Analyst

Exxon Nuclear Idaho, Box 2800

Idaho Falls, ID 83401

Jan Wirstad

Ergonomrad $\mathrm{AB}$

Box 205

S-651 02 Kar1stad

Sweden

Dr. John L. Woodward

Ecology and Environment

P.O. Box D

Buffalo, NY 14225

D. C. Webster

Union Carbide Corp.

South Charleston Tech Center

P.O. Box 8361

S. Charleston, WV 25303

Dr. David Worledge

EPRI

3412 Hillview Ave.

Palo Alto, CA 94303
John Wreathall

Battelle Columbus Division

505 King Avenue

Columbus, $\mathrm{OH} \quad 43201$

Jan Wright

Bronnoyvn 20

1315 Nesoya

Norway

Dr. W. V. Wright

Bldg. 773-42A, Rm 222

Savannah River Laboratory

Aiken, SC 29808

Jon Young

Energy Inc., Suite 201

18515 S. Central Place

Kent, WA 98031

Prof. Takeo Yukimachi

Dept. of Administrative Engineering

Keio University

Hiyoshi, Yokohama

223 Japan

Dr. Zeinab A. Sabri

Technology International Inc.

429 W. Airline Highway, Suite S

LaPlace, LA 70068

Jin Zhuorong

Dept. of Nuclear and Thermal-

Hydraulic Research and Design

Shanghai Nuclear Engineering

Research and Design Institute

P.O. Box 4027

Shanghai, China

Sandia National Laboratories:

3141 S. A. Landenberger (5)

3151 W. L. Garner

6226 G. J. Kolb

6400 D. J. McCloskey

6410 N. R. Ortiz

6412 A. L. Camp

6412 M. P. Bohn

6412 F. T. Harper

6412 D. M. Kunsman

6412 A. C. Payne, Jr.

6412 T. A. Wheeler

6412 D. W. Whitehead 
DIST - 13

Sandia Distribution (Continued):
6414 A. S. Benjamin
6415 F. E. Haskin
6420 J. V. Walker
6440 D. A. Dahlgren
6500 A. W. Snyder
6513 D. D. Carlson
7200 H. W. Schmitt
7220 R. R. Prairie
7222 G. T. Merren
7223 B. P. Chao
7223 R. G. Easterling
7223 D. P. Miller
7223 A. D. Swain
7223 L. M. Weston
7223 H. O. Whitehurst
8024 P. W. Dean
8433 O. H. Schreiber 


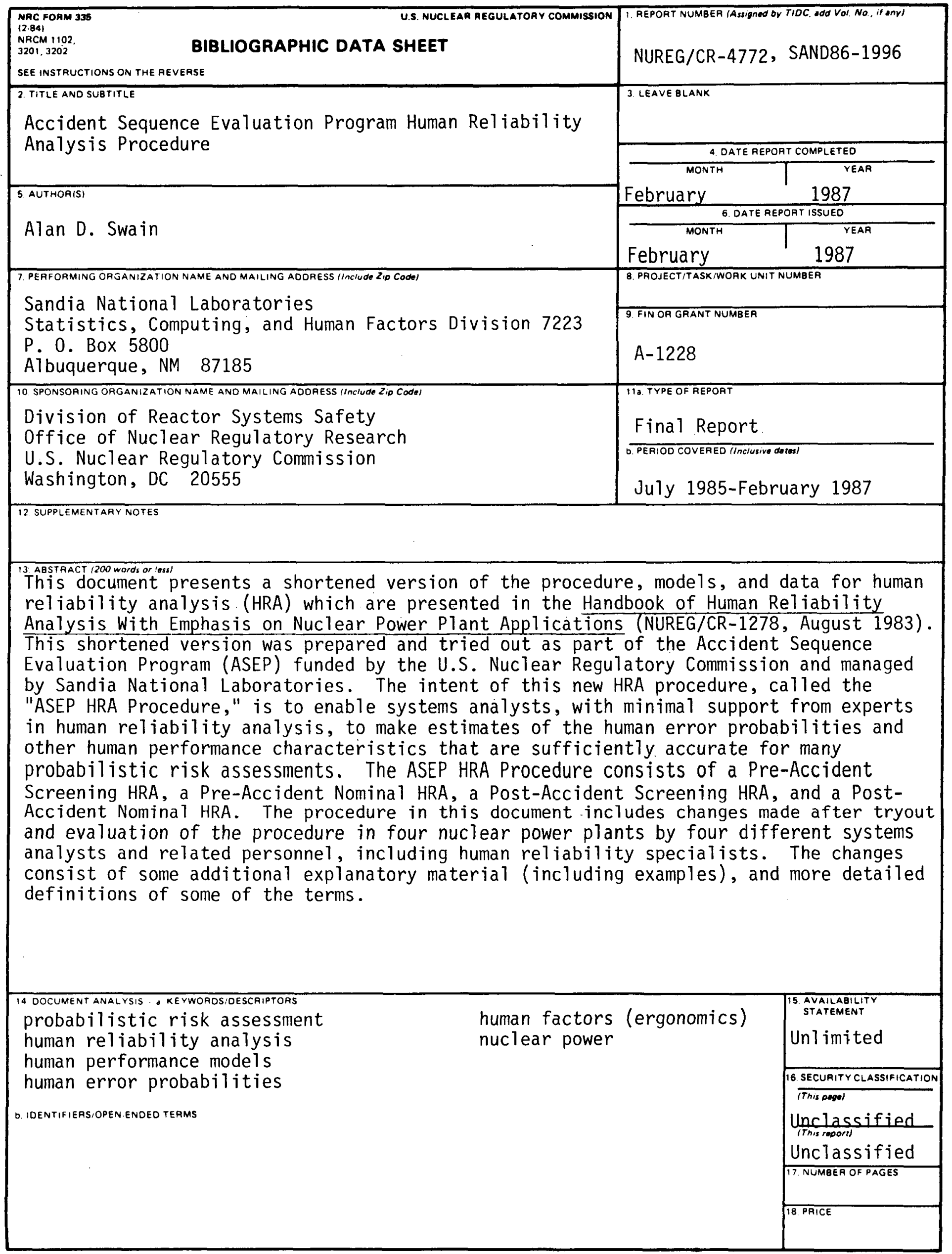

